\title{
Functional Anatomy of Macaque Striate Cortex. I. Ocular Dominance, Binocular Interactions, and Baseline Conditions
}

\author{
Roger B. H. Tootell, ${ }^{1}$ Susan L. Hamilton, ${ }^{1}$ Martin S. Silverman, ${ }^{2, a}$ and Eugene Switkes ${ }^{3}$ \\ 'Department of Psychology, University of California, Berkeley, California 94720, 'Department of Physiology, University of \\ California, San Francisco, California 94143, and ${ }^{3}$ Committee on Psychobiology, Division of Natural Sciences, University of \\ California, Santa Cruz, California 95064
}

\begin{abstract}
A series of experiments was carried out using ${ }^{14} \mathrm{C}-2-$ deoxyd-glucose (DG) in order to examine the functional architecture of macaque striate (primary visual) cortex. This paper describes the results of experiments on uptake during various baseline (or reference) conditions of visual stimulation (described below), and on differences in the functional architecture following monocular versus binocular viewing conditions.
\end{abstract}

In binocular "baseline" experiments, monkeys were stimulated either (1) in the dark, (2) with a diffuse gray screen, or (3) with a very general visual stimulus composed of gratings of varied orientation and spatial frequency. In all of these conditions, DG uptake was found to be topographically uniform within all layers of parafoveal striate cortex. In monocular experiments that were otherwise similar, uptake was topographically uniform within the full extent of the eye dominance strip, in all layers. Certain other visual stimuli produce high uptake in the blobs, and still another set of visual stimuli (including high-spatial-frequency gratings) produce highest uptake between the blobs at parafoveal eccentricities, even in an unanesthetized, unparalyzed monkey. Eye movements per se had no obvious effect on striate DG uptake. Endogenous uptake in the blobs (relative to that in the interblobs) appears higher in the squirrel monkey than in the macaque.

The pattern of DG uptake produced by binocular viewing was found to deviate in a number of ways from that expected by linearly summing the component monocular DG patterns. One of the most interesting deviations was an enhancement of the representation of visual field borders between stimuli differing from each other in texture, orientation, direction, etc. This "border enhancement" was confined to striate layers 1-3 (not appearing in any of the striate input layers), and it only appeared following binocular, but not monocular, viewing conditions. The border enhancement may be related to a suppression of DG uptake that occurs during binocular viewing conditions in layers $2+3$ (and perhaps layers 1 and

\footnotetext{
Received July 2, 1986; revised June 22, 1987; accepted Aug. 26, 1987.

We thank Russell L. De Valois for his generosity in supporting this long series of experiments. This work was supported by United States Health Service Grants EY-00014 and EY-02050 and National Science Foundation Grants BNS 82-02275 and BNS 78-86171.

Correspondence should be addressed to Roger B. H. Tootell at his present address: Department of Neurobiology, Harvard Medical School, 25 Shattuck St., Boston, MA 02115.

a Present address: Central Institute for the Deaf, $818 \mathrm{~S}$. Euclid Ave., and the Department of Anatomy and Neurobiology, Washington University, St. Louis, MO 63110.

Copyright (C) 1988 Society for Neuroscience $0270-6474 / 88 / 051500-31 \$ 02.00 / 0$
}

$4 B$ ), but not in layers $4 \mathrm{Ca}, 4 \mathrm{Cb}, 5$ or 6 . Another major class of binocular interaction was a spread of neural activity into the "unstimulated" ocular dominance strips following monocular stimulation. Such an effect was prominent in striate layer $\mathbf{4 C a}$, but it did not occur in layer $\mathbf{4 C b}$. This "binocular" spread of DG uptake into the inappropriate eye dominance strip in $4 \mathrm{Ca}$ may be related to the appearance of orientation tuning and orientation columns in that layer. No DG effects were seen that depended on the absolute disparity of visual stimuli in macaque striate cortex.

In a series of studies (see Tootell et al., 1988a-d, and unpublished observations), we report the results of functional anatomical experiments on macaque primary visual (striate) cortex. These studies utilize the ${ }^{14} \mathrm{C}$-2-deoxyglucose autoradiographic technique (Sokoloff et al., 1977) to investigate the functional architecture of cells responding to different visual stimuli. Each experimental manipulation was designed to test for specific responses to various stimulus parameters. These parameters include ocular dominance, orientation, spatial frequency, retinotopic specificity, color, contrast sensitivity, direction, and binocular interactions. By analyzing all the stimulus-specific results in a single, large-scale study in which uncontrolled differences were assiduously minimized, it has proven possible to chart the flow of different kinds of visual information through this critically important visual cortical area. In this, the first paper of the series, we describe functional anatomical studies of ocular dominance and binocular interactions. In addition, we describc techniques and report on cxperiments that provide baseline conditions relevant to all of the studies reported in the following papers (Tootell et al., 1988a-d).

Within striate cortex, cells that are predominantly sensitive to visual stimulation from one eye or the other are grouped together in ocular dominance columns. Among the various radially arranged systems in striate cortex, eye dominance columns have been the easiest to demonstrate with anatomical techniques, largely because information from each eye remains segregated until it reaches striate cortex (primarily in layer $4 \mathrm{C}$ ). The segregation of eye inputs is such a basic feature of the geniculorecipient layer $4 \mathrm{C}$ architecture that ocular dominance strips can be demonstrated with stains for Nissl substance (Haseltine et al., 1979), fiber stains (LeVay et al., 1975), and stains for fiber degeneration (Hubel and Wiesel, 1972), for transneuronal transport of tritiated amino acids (Wiesel et al., 1974), for monoclonal antibodies (Hockfield et al., 1983), HRP, and for cytochrome oxidase and other enzymes (e.g., Horton, 1984), as 
well as with functional measures, such as extracellular recording (Hubel and Wiesel, 1972, 1977) and deoxyglucose (DG) (Kennedy et al., 1975; Hendrickson and Wilson, 1979; Horton and Hubel, 1981; Tootell et al., 1982b).

Unfortunately, the purely anatomical techniques label eye dominance "columns" in only one or a few cortical layers. It has been possible to trace the anatomy of ocular dominance columns through the full cortical depth only with DG (a point made by Kennedy et al., 1975). As one might guess, there are some interesting aspects to the segregation of eye inputs beyond the input layers.

It is likely that ocular dominance columns serve to segregate information from each eye in radial, retinotopically specific bundles (from the geniculorecipient layer into subsequent striate layers) until it can be recombined into a binocular code that preserves fine-disparity information.

At least 2 arguments can be mustered to support this bit of teleology. The first argument relies on dircct physiological cvidence. Working with awake, behaving monkeys that viewed stimuli at different depths, Poggio and Fischer (1977) found a very strong correlation between the ocular dominance class of individual cells (tested monocularly) and their tuning to various binocular disparities. According to this evidence, zero-disparity cells generally receive strong inputs from both eyes, whereas near- and far-disparity cells usually receive strong excitatory input from only one eye. Because there is a strong relationship between the disparity tuning and the ocular dominance class of striate cells, and because the 2 functions are conceptually related, it is likely that ocular dominance and binocular disparity processing are related at a neural level as well.

The second argument is logical rather than empirical: information about object mismatch that is combined randomly in binocular cells cannot be retrieved later. Therefore, disparity coding must occur at the very first binocular stages. Since cells in extrastriate cortex all show some evidence of binocular input, we must look to striate cortex for the origins of disparity coding. Though other kinds of binocular coding are conceivable, the simplest to imagine is one in which neurons are tuned to different binocular disparities, for which there is much experimental evidence. One might expect the architecture for striate disparity processing to be highly organized because the computations involved in stereopsis are so massive (Marr and Poggio, 1979).

For these reasons, it would not be surprising to find some sort of organized functional architecture for binocular interactions, perhaps related to that for ocular dominance. Thus, one of the major aims of this study was to compare DG uptake following monocular stimulation to that seen in binocular conditions. Our rationale for this approach was that deviations from the sum of monocular DG activity in the binocular DG results might indicate disparity-related processing. As a necessary antecedent, we closely examined the topography of monocular dominance strips, so that we could adequately define deviations from a sum of monocular activity in the binocular conditions.

An additional goal of this study was to carefully define the pattern of DG uptake in several reference (or "baseline") conditions of binocular visual stimulation, especially with reference to the cytochrome oxidase blobs observed in the upper striate layers (Hendrickson et al., 1981; Horton and Hubel, 1981) (see Fig. 1). This is an area in which the data are somewhat clouded, so we went to some lengths to clarify the role of the metabolically rich cytochrome oxidase blobs relative to DG measures of func- tional activity in macaque striate cortex. This was vital in interpreting the results from our accompanying DG studies.

\section{Materials and Methods}

Over the 5 year duration of this series of studies, 56 macaque monkeys and 1 squirrel monkey were used. Macaque monkeys of several different species were used: $28 \mathrm{M}$. fascicularis, $15 \mathrm{M}$. arctoides, $7 \mathrm{M}$. assamensis, $5 \mathrm{M}$. nemestrina, and $1 \mathrm{M}$. radiata. Although the size and intercolumnar spacing of striate tissue varied between species with different body and brain sizes (see below), we saw very few interspecies differences in other aspects of DG results. Because we saw so few architectural differences (and because we were able to factor out those minor variations that appeared to be species-specific), the diverse range of species sampled permitted us to generalize our results more meaningfully to other Old World primates, such as man.

Surgical procedure. Two macaque monkeys were injected with DG while in an unanesthetized, unparalyzed state; further details from these cases are given later (cases 16, 51). The remaining 54 macaques and the 1 squirrel monkey were implanted with a plastic headset under deep surgical anesthesia $(1 \mathrm{mg} / \mathrm{kg}$ diazepam, $15-30 \mathrm{mg} / \mathrm{kg} / \mathrm{hr}$ ketamine hydrochloride, and $5-25 \mathrm{mg} / \mathrm{kg} / \mathrm{hr}$ sodium pentobarbital) in sterile conditions at least a week before the main DG experiment. The prior implantation of headsets allowed head restraint without the use of painful ear bars during the subsequent DG experiment, as well as obviating the need for acute surgical excision of skin and muscle tissue overlying striate cortex.

On the day of the DG experiment, monkeys were initially anesthetized with ketamine hydrochloride $(20 \mathrm{mg} / \mathrm{kg})$, supplemented as needed with sodium pentobarbital, additional ketamine hydrochloride, and atropine sulfate. A bar was attached to the plastic headset so that the head could be held rigidly in the stereotaxic apparatus. The eyelids were kept carefully shut during this stage to prevent drying of the outer corneal layers. The radial or the cephalic vein was cannulated to allow a constant intravenous administration of Ringer's solution (with or without $3 \%$ lactose), as well as of various drugs. Animals were then intubated with rubber endotracheal tubes (Rusch) coated with Zyljectin (a long-lasting local anesthetic), paralyzed (10-20 $\mathrm{mg} / \mathrm{kg} / \mathrm{hr}$ gallamine triethiodide and $0.1-0.3 \mathrm{mg} / \mathrm{kg} / \mathrm{hr} d$-tubocurarine) and artificially respirated. Expired $\mathrm{CO}_{2}$ and the electrocardiogram were monitored and kept within normal physiological limits.

Although acute surgical invasion was either absent or minimal in this preparation, profound analgesia was maintained throughout the experiment with $0.5 \mathrm{mg} / \mathrm{kg} / \mathrm{hr}$ morphine sulfate, and light supplemental anesthesia was maintained during all but the final hour of the experiment with a $75 / 25 \%$ mixture of nitrous oxide and oxygen. In combination with mild preanesthetics and muscle paralysis, almost identical anesthetic regimens $\left(70 / 30 \% \mathrm{~N}_{2} \mathrm{O}\right.$ and morphine in doses equal to those used in the present study) have been shown to be fully adequate for abdominal surgery in humans (Stanski et al., 1978). If any environmentally related change in heart rate was obscrved, the ancsthesia level was supplemented by sodium pentobarbital. Such changes were tested for often, and rarely observed. During the DG infusion (well past the time when any minor acute surgical invasion took place), room air was titrated in with the nitrous oxide/oxygen mixture (often 1:1) because $75 / 25 \% \mathrm{~N}_{2} \mathrm{O}$ was found to decrease the contrast of the DG results. During this time, barbiturate and morphine dosage levels remained constant or were slightly increased. A heating pad thermostatically controlled by a rectal probe kept core temperature at $37-38^{\circ} \mathrm{C}$.

The eyelids were retracted, and the cornea was locally anesthetized with a $0.5 \%$ solution of proparacaine hydrochloride (Opthaine). Hard or gas-permeable contact lenses of the appropriate curvature were placed on the cornea and accommodation paralyzed with atropine sulfate infused below the contact lens near the sclera. The fovea was back-projected with a reversible ophthalmoscope and $3 \mathrm{~mm}$ artificial pupils wcrc carefully centered on the optic axis. Supplemental lenses were added in front of the contact lens in order to bring the stimulus screen into focus at a distance of either 57 or $87 \mathrm{~cm}$. The application of contact lenses and subsequent optical procedures were delayed for as long as possible in the DG experiment in order to minimize oxygen deprivation of the corneal epithelium.

In some experimental cases, we recorded from foveal striate cortex with microelectrodes, and used those foveal receptive fields as an index of binocular convergence and to locate the foveal projection in the visual 


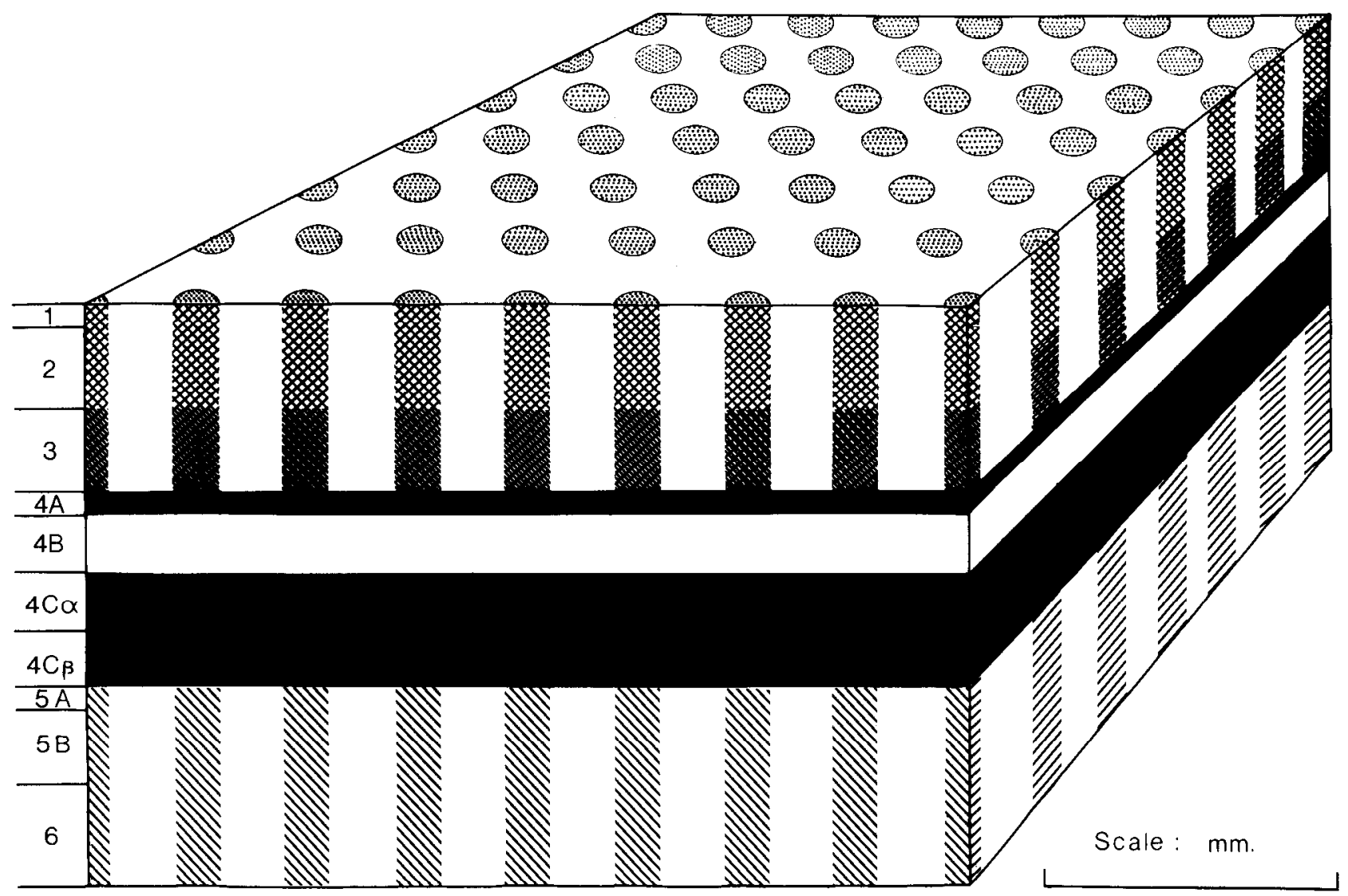

Figure 1. Diagram of the cytochrome oxidase architecture in macaque striate cortex. The cytochrome oxidase "blobs" (also described as "puffs" or "patches") are found in all layers outside of layer 4 and marginally in layer 4B. The various stippling patterns in the diagrammatic blobs in each of the indicated layers give some idea of the corresponding contrast of the blobs in vivo. The blobs are most obvious in layer 3 , less so in layer 2 , and fainter in layers 1,5, and 6. In some cases, the blobs are marginally visible, and in others, they are invisible, as in layer 4B (indicated by a lack of slippling). Suale bar, $1 \mathrm{~mm}$.

field. In these cases, a small bone plate overlying foveal striate cortex was carefully removed after infusion of $8-10 \mathrm{ml} / \mathrm{kg}$ of $20 \%$ mannitol (Sigma), $0.1 \mathrm{mg} / \mathrm{kg}$ dexamethasone, and levels of barbiturate sufficient to maintain surgical levels of anesthesia. The bone plate was removed before paralysis, so that the state of anesthesia could be monitored accurately and kept at surgical levels. Usually, the endosteal layer of the dura overlying foveal striate cortex was pulled apart over a few square millimeters so that the electrode needed only to pierce the meningeal dural layer. A glass-insulated tungsten microelectrode (after Merrill and Ainsworth, 1972) was driven by a microdrive into foveal striate cortex under direct visual control, and single- or multiple-unit activity was recorded.

Visual stimuli. Visual stimuli were presented on a Tektronix color monitor (in early experiments, model 654, and in later experiments, model 690 ) driven by a modified Lexidata image processor and a NOVA $4 \mathrm{X}$ computer. Mean luminance ranged from 4 to $104 \mathrm{~cd} / \mathrm{m}^{2}$, depending on the stimulus used. At this luminance, individual pixels could not be resolved at either of the 2 distances $(57$ and $87 \mathrm{~cm}$ ) with the model 690 , but were barely visible at $57 \mathrm{~cm}$ on the model 654 to an emmetropic experimenter. When pixel visibility on the model 654 could be problematic, and when spatial resolution was not critical in a particular experiment, the eyes were deliberately defocused by a few diopters. Except as noted, visual stimulation during DG infusion was done with the room lights on. A large white panel with a circular aperture in the center was normally attached to the front of the monitor to minimize possiblc luminanec artifacts at the peripheral border of the stimulus screen. Screen diameter, viewed through the aperture, was $27^{\circ}$ at $57 \mathrm{~cm}$ and $18^{\circ}$ at $87 \mathrm{~cm}$.

The visual display system allowed independent control over stimulus color, orientation, contrast, position, size, and shape (waveform and spatial frequency for 1-dimensional patterns), velocity/drift rate, etc. In the simplest experiments, a single visual parameter was held constant across the entire screen, while all other visual parameters were varied. For instance, the orientation of a pattern might be held constant, while the spatial frequency, position, drift rate, and color of the pattern were varied over time.

Stimuli varying along any of these dimensions could be presented in a single region covering the whole screen (as described above) or in combination with other stimuli in as many as 4-8 different regions of the screen (Switkes et al., 1986). When the split-screen format was employed, stimuli in each of the screen regions differed from one another only in a single parameter (the independent variable). When appropriately centered in the monkey's visual field, such a split-screen stimulus allowed a within-animal comparison of the effect of cach visual stimulus parameter on resultant DG patterns. This allowed a definitive confirmation (and, in a very few cases, a sensible revision) of conclusions drawn from between-animal comparisons. The split-screen format was used in 28 cases; the remaining animals were shown sets of stimuli that covered the full extent of the screen. A complete cycle of controlled stimulus parameters took $0.03-12$ min to complete, depending on the total number of parameters varied in each stimulus. Periods of specific visual stimulation and active DG uptake varied from 25 to $50 \mathrm{~min}$. The shorter stimulus durations were used in order to minimize the possible confounding effects of minor eye movements (see below). Specific visual stimuli are described case by case in Results. A summary list of all cases and stimuli appears in Table 1.

Ocular alignment and general experimental procedure. In many electrophysiological studies of binocular interactions, the presence of minor eye movements has made the binocular disparity both difficult to specify and to maintain at a single value. However, in the present study we believe that artifacts due to eye drift were not a problem. If significant eye movements had occurred in the present set of DG experiments, the extent and the direction of the eye movements would have been obvious as a blurred image in the retinotopic DG representations of the stimulus 
border within striate cortex. We never saw such a blurring of the DG pattern in the brain. In direct tests (described in the retinotopic section), the falloff of DG uptake at the representation of a stimulus border (halfamplitude, half-width) had a horizontal extent corresponding to about $2^{\prime}$ of arc in striate layer $4 \mathrm{Cb}$. We can thus conclude that any eye movements that may have occurred during these experiments were less than or equal to 2' of arc. The lack of obvious eye movements in these DG studies undoubtedly results from (1) the high doses of paralytic used in these experiments, and (2) the fact that most of the DG uptake occurs within 10 min after injection. In single-unit experiments, the sampling times can be much longer; thus the cumulative effect of minor eye movements becomes more of a problem.

When visual stimuli were presented in retinotopically distinct regions (as in split-screen experiments), it was necessary to center the visual display screen very carefully in the monkey's visual field to insure that the different stimuli would be equated for eccentricity. In binocular experiments, it was also necessary to carefully align the 2 visual-field centers in order to prevent diplopia. When experimental diplopia occurred, it was obvious as a "double exposure" in the DG autoradiographs, weeks after the experimental diplopia occurred. During the experiments themselves, we were able to coordinate visual-field position with stimulus screen position by adjusting a variable Risley prism in front of one eye. However, it proved unexpectedly difficult to measure the center of the visual field exactly.

Two methods were used to assess foveation. In the first we backprojected the apparent foveal reflex with a reversible ophthalmoscope. Because of residual optomechanical errors in our reversible ophthalmoscope, the most reliable way of doing this involved mounting the ophthalmoscope in 2 different positions rotated $180^{\circ}$ from each other, and then taking the difference between the 2 readings to be the "real" foveal projection. This method proved adequate for many experiments. However, the large magnification of the visual field in foveal striate cortex resulted in visual stimulus borders in the autoradiographs that veered off from that area in a quite disconcerting way following even small $\left(1 / 8^{\circ}-1 / 4^{\circ}\right)$ errors in back-projection.

In general, microelectrode recording of foveal receptive fields furnished a more sensitive indicator of the foveal projection (see above for details of microelectrode placement). When binocular convergence was assessed from microelectrode recordings, an attempt was made to sample activity from cell groups in which the excitatory inputs from each eye were equally balanced. These excitatory-balanced neurons are generally tuned at or near zero disparity (Poggio and Fischer, 1977; Ferster, 1981; Poggio and Talbot, 1981).

In 25 monkeys, stimuli were presented monocularly. In the remaining binocularly stimulated animals, the foveal projection of both eyes was brought to converging points on the stimulus screen by adjusting a Risley rotary prism. However, if cells tuned to a common binocular disparity are grouped together in striate cortex, it is possible that visual stimulation at a constant binocular disparity might inadvertently produce artifactual patterns of uptake in disparity-specific subregions of striate cortex during our experiments. Therefore, a control for this was employed in some animals. In these cases, horizontal disparity was continuously varied over $1^{\circ}$ of visual angle during periods of DG uptake. This range is adequate to encompass the disparity specificity of most striate cells (Poggio and Fischer, 1977; Poggio and Talbot, 1981).

When ocular alignment had been optimized, the animal was injected with 12.5-50 $\mu \mathrm{Ci} / \mathrm{kg}$ DG (Amersham, New England Nuclear, Pathfinders, or American Radiochemicals) reconstituted in Ringer's solution $(\mathrm{pH} \sim 7.6)$.

At this juncture, an additional stimulus-related control should be described. As mentioned earlier, serial presentation of a full complement of patterns representing all stimulus parameters (e.g., all orientations, all spatial frequencies, etc.) could take several minutes to complete. Recall that the uptake of DG is markedly nonlinear over time (Sokoloff et al., 1977); much of the uptake takes place within the first $10 \mathrm{~min}$. Thus, conventional single-bolus injections might well produce DG patterns that are inadvertently biased towards those orientations, colors, etc., that were presented shortly after the injection. To minimize this possibility, we injected DG in a continuous fashion over 10-15 min (a length of time that included all possible stimulus combinations) and randomized the order of stimulus variables. In those few cases in which a single visual parameter was varied quickly, DG was injected in a single pulse.

As a control for possible differences in DG uptake between paralyzed and unparalyzed states, 2 unparalyzed, unanesthetized macaques were injected with DG, intraperitoneally, while (1) in a cage in the dark, or (2) manually restrained, viewing the inside of a specially constructed hemispheric panel. Subsequent histological processing of tissue from these animals was identical to that used on the paralyzed, anesthetized animals.

Histology. Thirty to forty-five minutes after the end of DG infusion, monkeys were killed by injection with $50-75 \mathrm{mg} / \mathrm{kg}$ sodium pentobarbital and sodium heparin. When pronounced decreases in electrocardiograms and expired $\mathrm{CO}_{2}$ became manifest, the animals were perfused transcardially with a $0.1 \mathrm{M}$ phosphate buffer solution ( $\mathrm{pH} 7.4$ ) containing $10 \%$ sucrose. A short (about $40 \mathrm{sec}$ ) perfusion with a $10 \%$ sucrose phosphate-buffered solution containing 3.5\% formalin followed, and then a rinse with the original solution. The animal was decapitated, the brain was removed from the skull, and the occipital operculum from each hemisphere was separated from the rest of the brain at the "stem" of the operculum. This tissue contained regions of striate cortex representing eccentricities from fovea to about $20^{\circ}$ (Daniel and Whitteridge, 1961 ) and somewhat more central regions of V2 (Gattass et al., 1981). The dissected cortical tissue was laid surface down on a silicone-coated slide. Because of the light fixation, the operculum spread out quite flat on the slide owing entirely to passive mechanical constraints; the tissue was not squashed between 2 flat objects. The tissue was then frozen by apposition to a block of metal chilled to about $-70^{\circ} \mathrm{C}$. The tissue was then stored at $-70^{\circ} \mathrm{C}$ until it could be cut in a cryostat. Further details of the flattening procedure can be found in Tootell and Silverman (1985).

Several days later, tissue was mounted in an International Harris (IEC) cryostat and cut at $-17^{\circ}$ to $-22^{\circ}$. Except in a very few cases, sections were cut parallel to the flattened cortical surface in 30-40 micron sections. To eliminate artifact, sections were cut very slowly and reflattened on the cryostat knife blade with a cold paintbrush. They were then picked up on $\# 1.5$ coverslips (previously subbed in 1\% gelatin and $0.05 \%$ chrome-alum) and heated on a hot plate at about $50^{\circ} \mathrm{C}$ until just dry. Autoradiographs were made by mounting the sections on poster board and sandwiching the sections against $\mathrm{x}$-ray film (Kodak SB-5 or, for higher resolution, Kodak Min- $\mathrm{R}$ ) in cassettes stored at $-70^{\circ} \mathrm{C}$. In many of the cassettes we included methyl methacrylate ${ }^{14} \mathrm{C}$ standards (Amersham 196363) so as to be able to convert optical density values in the autoradiographs back to relative levels of DG uptake. Autoradiographic exposure time ranged from 2 to 12 weeks, depending on DG dosage, section thickness, and $\mathrm{x}$-ray film type. The $\mathrm{x}$-ray film was developed with Kodak GB-X developer.

After autoradiography, sections were dipped in a $10 \%$ sucrose phosphate buffer solution, air-dried, and then fixed to the subbed coverslips in a $2 \%$ gluteraldehyde, $10 \%$ sucrose phosphate-buffered solution. Sections prepared in this way adhered tightly to the coverslips during subsequent staining procedures.

Most tissue was stained for cytochrome oxidase (cytox). In order to increase the contrast of the stain, tissue sections were first soaked in a weak cobalt chloride solution $(275 \mathrm{mg} \mathrm{CoCl} / \mathrm{liter}$ in a Tris buffer with $10 \%$ sucrose, $\mathrm{pH} 7.6$ ) for $10 \mathrm{~min}$. Four successive $5 \mathrm{~min}$ rinses in a $10 \%$ sucrose phosphate-buffered solution ( $\mathrm{pH} 7.4$ ) removed excess cobalt chloride. Sections were then incubated in the reaction solution $[50 \mathrm{mg}$ diaminobenzidine tetrachloride (DAB), $7.5 \mathrm{mg}$ cytochrome $\mathrm{C}, 2.0 \mathrm{mg}$ catalase, $0.25 \mathrm{ml}$ dimethyl sulfoxide (DMSO) $/ 100 \mathrm{ml}$ of $0.1 \mathrm{M}$ phosphate-buffered solution, with $10 \%$ sucrose, $\mathrm{pH} 7.5$ ] for $1-6 \mathrm{hr}$ at $37-$ $40^{\circ} \mathrm{C}$. The reaction solution was oxygenated and stirred periodically. When stained, the sections were run through an ascending series of alcohols, followed by xylenes, and then coverslipped with Permount. A few other sections were stained with thionin.

Laminar distinctions. Laminar distinctions were generally made on the basis of staining for cytochrome oxidase (Blasdel and Fitzpatrick, 1984; Horton, 1984; Livingstone and Hubel, 1984) (see Fig. 1), supplemented by Nissl stains or by reference to the DG itself in layers that were not clearly differentiable with the enzyme stain. The first and second $40 \mu \mathrm{m}$ sections were presumed to be from layer 1 , since the section plane was parallel with the cortical surface. When it was important to define the layer 1-2 border exactly, this distinction was made on the basis of the great increase in the number of Nissl-stained cell bodies in layer 2 relative to layer 1 . The layer $2-3$ border was often crudely estimated by keeping track of section position within a histological series. However, a few visual stimuli (e.g., gratings of high spatial frequency) produce DG patterns that are markedly darker below a depth that is roughly halfway between the top of layer 2 and the bottom of layer 3 . In such cases, this DG border was taken to indicate the layer 2-3 bordcr. In many animals the cytochrome oxidase blobs became 


\begin{tabular}{|c|c|c|c|}
\hline $\begin{array}{l}\text { Case } \\
\text { no. }\end{array}$ & Species & $\begin{array}{l}\text { Viewing } \\
\text { conditions }\end{array}$ & Stimulus \\
\hline 1 & M. fas. & Bin & Spatially diffuse red-green flicker \\
\hline 2 & M. fas. & Bin & Spatially diffuse black-white flicker \\
\hline 3 & M. nem. & Bin & Spatially diffuse red-gray flicker \\
\hline 4 & M. fas. & Bin & Split-field (2-sector), spatially diffuse red-gray vs luminance-varying flicker \\
\hline 5 & M. arc. & Bin & Spatially diffuse blue-gray flicker \\
\hline 6 & M. fas. & Bin & Spatially diffuse red-gray flicker \\
\hline 7 & M. fas. & Bin & Spatially diffuse black-white flicker \\
\hline 8 & M. fas. & Bin & Horizontal, var. sp. freq. square-wave grating \\
\hline 9 & M. arc. & Bin & Ring and ray stimulus (checks) \\
\hline 10 & M. fas. & Bin & Diffuse gray screen (no flicker) \\
\hline 11 & M. arc. & Mon & Ring and ray stimulus (solid) \\
\hline 12 & M. arc. & Bin & Var. orientation, var. sp. freq., var. color, square-wave grating \\
\hline 13 & M. arc. & Mon & Ring and ray stimulus (checks) \\
\hline 14 & M. arc. & Bin & Vertical, var. sp. freq. square-wave grating (var. disparity) \\
\hline 15 & M. arc. & Mon & Var. orientation, var. sp. freq. square-wave grating \\
\hline 16 & M. fas. & Bin & Unparalyzed, unrestrained in the dark \\
\hline 17 & M. fas. & Mon & Var. orientation, var. sp. freq., red-gray square-wave grating \\
\hline 18 & M. fas. & Mon & Ring and ray stimulus (checks) \\
\hline 19 & M. fas. & Bin & Var. orientation, 6.5 cycle/deg sinusoidal grating (var. disparity) \\
\hline 20 & M. fas. & Bin & Var. orientation, 1 cycle/deg sinusoidal grating (var. disparity) \\
\hline 21 & M. fas. & Mon & Horizontal, $6.5 \mathrm{cycle} / \mathrm{deg}$ sinusoidal grating \\
\hline 22 & M. fas. & Mon & Split-field (2-sector), one vs var. orientation, var. sp. freq. sinusoidal grating \\
\hline 23 & M. arc. & Mon & $\begin{array}{l}\text { Split-field ( } 2 \text {-sector), color vs luminance-varying sinusoidal grating, var. orientation, var. sp. } \\
\text { freq. (with blankout rings) }\end{array}$ \\
\hline 24 & M. fas. & Bin & Split-field (2-sector), 7 cycle/deg vs var. sp. freq. sinusoidal grating \\
\hline 25 & M. fas. & Bin & $\begin{array}{l}\text { Split-field ( } 8 \text {-sector), spatially diffuse color-gray flicker (red vs green vs yellow vs blue/ } \\
\text { gray } \times 2 \text { ) }\end{array}$ \\
\hline 26 & M. fas. & Mon & Vertical meridian, var. orientation, var. sp. freq. square-wave grating \\
\hline 27 & M. fas. & Mon & Split-field (4-sector), 6.5 vs 1 cycle/deg color vs luminance-varying sinusoidal grating \\
\hline 28 & M. fas. & Bin & Vertical, 6.6 cycle/deg, sinusoidal grating (var. disparity) \\
\hline 29 & M. arc. & Mon & $\begin{array}{l}\text { Split-field (4-sector), } 6.5 \text { vs } 1 \text { cycle/deg, color vs luminance-varying sinusoidal grating, var. } \\
\text { orientation }\end{array}$ \\
\hline 30 & M. arc. & Mon & Split-field (2-sector), Horizontal, 7 vs 1 cycle/deg sinusoidal grating (with blankout rings) \\
\hline 31 & M. fas. & Bin & Horizontal, 7 cycle/deg sinusoidal grating \\
\hline 32 & M. fas. & Bin & Vertical, 0.9 cycle/deg sinusoidal grating (var. disparity) \\
\hline 33 & M. fas. & Mon & $\begin{array}{l}\text { Split-field ( } 3 \times 2 \text { sector), spatially diffuse color-gray flicker (red vs green vs blue/gray), } \\
\text { equal saturation vs equal purity }\end{array}$ \\
\hline 34 & M. ass. & Mon & Split-field (4-sector), 4.1 vs 0.7 cycle/deg, color vs luminance-varying sinusoidal grating \\
\hline 35 & M. rad. & Mon & $\begin{array}{l}\text { Split-field (4-sector), } 8 \text { vs } 18 \text { vs } 40 \text { vs } 100 \% \text { contrast square-wave grating, horizontal, var. } \\
\text { sp. freq. }\end{array}$ \\
\hline 36 & M. ass. & Mon & $\begin{array}{l}\text { Split-field (4-sector), } 4.1 \text { vs } 0.7 \text { cycle/deg, color vs luminance varying, sinusoidal grating, } \\
\text { var. orientation }\end{array}$ \\
\hline 37 & $M$. ass. & Bin & Var. orientation, var. sp. freq. square-wave grating (var. disparity) \\
\hline 38 & M. fas. & Mon & $\begin{array}{l}\text { Split-field (4-sector), red vs blue vs green/gray vs black/white, } 2.7 \text { cycles/deg sinusoidal } \\
\text { grating, var. orientation (blankout rings) }\end{array}$ \\
\hline 39 & M. ass. & Bin & Split-field (2-sector), 4.4 vs 0.7 cycle/deg sinusoidal grating, var. orientation \\
\hline 40 & M. nem. & Bin & Split-field (2-sector), 7 vs 1 cycle/deg sinusoidal grating, var. orientation \\
\hline 41 & M. arc. & Mon & $\begin{array}{l}\text { Split-field (6-sector), spatially diffuse color-gray flicker (red vs green vs blue/gray), equal } \\
\text { saturation vs equal purity }\end{array}$ \\
\hline 42 & M. fas. & Bin & Var. orientation, var. sp. freq. square-wave grating \\
\hline 43 & M. ass. & Mon & $\begin{array}{l}\text { Split-field (4-sector), } 8 \text { vs } 18 \text { vs } 38 \text { vs } 100 \% \text { contrast, var. sp. freq. square-wave grating, } \\
\text { oblique orientation }\end{array}$ \\
\hline 44 & M. arc. & Mon & $\begin{array}{l}\text { Split-field ( } 2 \text {-sector), } 4.8 \text { vs } 0.7 \text { cycle/deg square-wave grating, oblique orientation (with } \\
\text { blankout rings) }\end{array}$ \\
\hline 45 & M. ass. & Bin & $8 \%$ Contrast, var. orientation, var. sp. freq. square-wave grating \\
\hline 46 & M. ass. & Bin & $\begin{array}{l}\text { Split-field (2-sector), } 180^{\circ} \text { vs } 360^{\circ} \text { directions, var. orientation, var. sp. freq. square-wave } \\
\text { grating }\end{array}$ \\
\hline
\end{tabular}




\begin{tabular}{|c|c|c|c|}
\hline $\begin{array}{l}\text { Case } \\
\text { no. }\end{array}$ & Species & $\begin{array}{l}\text { Viewing } \\
\text { conditions }\end{array}$ & Stimulus \\
\hline 47 & M. fas. & Bin & Random-dot pattern, one direction \\
\hline 48 & M. arc. & Mon & $\begin{array}{l}\text { Split-field (4-sector), } 0.1 \text { vs } 2-4.4 \text { cycle/deg red-gray vs } 0.1 \text { cycle/deg black-white sinusoidal } \\
\text { grating, var. orientation }\end{array}$ \\
\hline 49 & M. arc. & Bin & Split-field (2-sector), 4.4 vs 1 cycle/deg sinusoidal grating, var. orientation \\
\hline 50 & M. arc. & Bin/Mon & $\begin{array}{l}\text { Split-field ( } 2 \text {-sector), binocular vs monocular, var. sp. freq. sinusoidal grating, var. } \\
\text { orientation }\end{array}$ \\
\hline 51 & M. fas. & Bin & 7.5 cycle/deg square-wave grating, var. orientation (hand-held, unparalyzed) \\
\hline 52 & M. nem. & Mon & Horizontal, 0.5 cycle/deg, stationary, counterphased square-wave grating \\
\hline 53 & M. nem. & Mon & $\begin{array}{l}\text { Split-field (2-sector), red-gray vs red-black, } 6.5 \text { cycles/deg square-wave grating, var. } \\
\text { orientation }\end{array}$ \\
\hline 54 & M. fas. & Bin & 6.5 cycle/deg red-gray square-wave grating \\
\hline 55 & M. nem. & Bin & Split-field (2-sector), 6.5 vs 1.5 cycles/deg sinusoidal grating, var. orientation \\
\hline 56 & M. fas. & Bin & Random-dot pattern, var, directions \\
\hline
\end{tabular}

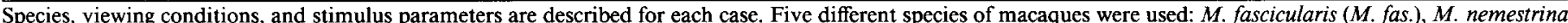

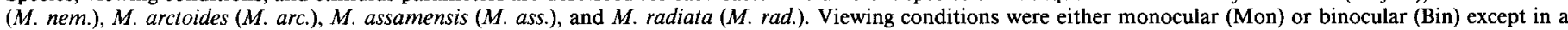

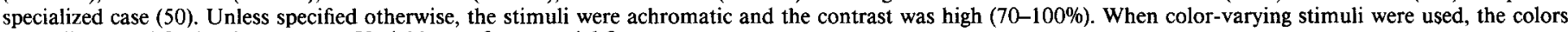
were all equated for luminance. var., Variable; sp. freq., spatial frequency.

noticeably darker at this depth; this further justifies a distinction between the 2 layers.

Layer $4 \mathrm{~A}$ was taken to be the very thin band of tissuc that stains dark and reticulated in a cytochrome oxidase reaction (Horton, 1984). Though the Nissl-defined 4A extends below this boundary (Blasdel and Fitzpatrick, 1984), all the DG patterns we see appear to be thin and coincident with the enzyme (rather than the Nissl)-staining distinctions. Layer $4 \mathrm{~B}$ is coincident with the layer of light cytochrome oxidase staining between $4 \mathrm{~A}$ and $4 \mathrm{Ca}$. The upper boundary of layer $4 \mathrm{Ca}$ is coincident with the upper boundary of dark cytochrome oxidase staining, or slightly above it.

Several types of visual stimulation produce patterns of DG uptake that change abruptly and obviously midway through the dark cytox band that characterizes almost all of layer $4 \mathrm{C}$ : we take this to be the $4 \mathrm{Ca}-4 \mathrm{Cb}$ border. Among the stimuli that produce abrupt DG changes between layers $4 \mathrm{Ca}$ and $4 \mathrm{Cb}$ are grating stimuli shown at a single orientation, luminance-modulated gratings of a low spatial frequency, spatially diffuse color stimuli, and luminance-modulated stimuli of low $(<10 \%)$ contrast. These laminar differences in DG effect are generally predictable from functional differences in the magnocellular and parvocellular LGN layers. Since layer $4 \mathrm{C}$ has been subdivided on the basis of a segregation of inputs from magnocellular and parvocellular LGN layers about midway through the dark cytox band (Livingstone and Hubel, 1984), the present DG subdivision is almost certainly coincident with previous conventions. We saw no DG evidence for subdivisions within layers $4 \mathrm{Ca}$ or $4 \mathrm{Cb}$.

In response to a few visual stimuli (e.g., certain magnocellular-specific stimuli sparing $4 \mathrm{Cb}$ ), layer $5 \mathrm{~A}$ is visible as a thin band of high $D G$ uptake immediately below layer $4 \mathrm{Cb}$. Generally, layers $5 \mathrm{~A}$ and $5 \mathrm{~B}$ are not differentiable immediately below layer $4 \mathrm{Cb}$. Layer $5 \mathrm{~B}$ (and sometimes layer $5 \Lambda$ ) is almost always lighter than layer 6 in the DG material. Sometimes layer 6 is darker than layer 5 in the cytox-stained material. There was no DG evidence for subdivisions within layer 6 .

Densitometric analysis. Much useful information could be obtained from the material by simply comparing cytox-stained sections to DG autoradiographs of those same sections on a light box. However, for a more quantitative analysis, both the cytox-stained section and the autoradiograph were densitized for computer analysis using a Perkin-Elmer flying spot microdensitometer. Normally an array of $512 \times 480$ density values was sampled in $25 \mu \mathrm{m}$ steps.

Two general kinds of densitometric analysis were performed: a laminar and a topographic one. Because of resolution problems inherent in ${ }^{14} \mathrm{C}$ radiography (and because most of our sections were cut tangentially), we did our laminar analysis in sections cut near-parallel to the cortical surface, rather than perpendicular to the surface. The rationale for this is as follows: The radiographic spread from a point source of ${ }^{14} \mathrm{C}$ has a width (at half-amplitude) of about $100 \mu \mathrm{m}$ (Goochee et al., 1980), which is about the thickness of some striate layers. In sections cut perpendicular to the laminae, densitometric measurements of each lamina will thus be inaccurate in the thinner striate layers and near the borders of all laycrs. Onc can compensatc for this problem by making density measurements across sections in which the sectioning plane is almost parallel to the cortical layers. In these almost-tangential sections, the autoradiographic densities in even the thinnest striate layers are accurate and clearly resolvable: large expanses of laminae that are adjacent in vivo are neatly abutted within a given section because we are sectioning nearparallel with the laminae. Furthermore, correction can be made for periodic (columnar) variations within a layer much more accurately in sections such as this, in which the density variation across a whole column can be seen clearly. Densities measured within a given layer in different sections were indistinguishable. In the laminar analyses, all optical densities were converted to normalized levels of DG uptake via radioactive standards.

An important goal of the present study was to relate stimulus-specific variations in DG uptake to the positions of the cytochrome oxidasc blobs. Except in a single case, the DG-cytox comparison was made in layer 3 , since the cytox blobs are most obvious in that layer, and the DG-cytox relationship is often similar in the upper and lower layers. Some operculae have a deep fissure down the middle (the ectocalcarine fissure) that obscures the topography near the anterior and ventral operculum. For this reason, the DG and cytox topographies were almost always compared just posterior to the position of this fissure, corresponding to eccentricities of about $3^{\circ}-4^{\circ}$.

Quantitative comparisons of the DG-cytox topography were made by first aligning digital versions of the densitized section and the autoradiograph on a color monitor. Sections could be aligned to the nearest $25 \mu \mathrm{m}$. Both images were spatially filtered with a difference-of-Guassians filter to eliminate high- and low-spatial-frequency artifacts. Density levels could then be compared in color-coded displays of superimposed DGcytox topography and in histograms of DG and cytox density at each pixel. This analysis was based on optical density rather than on radioactive uptake, since no comparable standards have been developed that relate optical density of the cytox stain to cytox concentration. Full details of the topographical DG-cytox analysis can be found in Switkes et al. (1986).

Other densitometric analyses were 1-dimensional. In these cases, densities were sampled within a given layer through an aperture of variable size, moved at various step sizes along a line. When aperture size was small, data from 1-dimensional scans were sometimes smoothed by averaging adjacent data points.

When ${ }^{14} \mathrm{C}$ standards were necessary (as in comparing absolute levels of DG uptake), autoradiographic density of the standards was sampled, along with density values from the tissue. Generally, both sets of densities were taken from the same sheet of $x$-ray film. A curve was fitted through the 8 standard values and tissue densities were adjusted accordingly. Because different doses of DG (and slightly different perfusion 


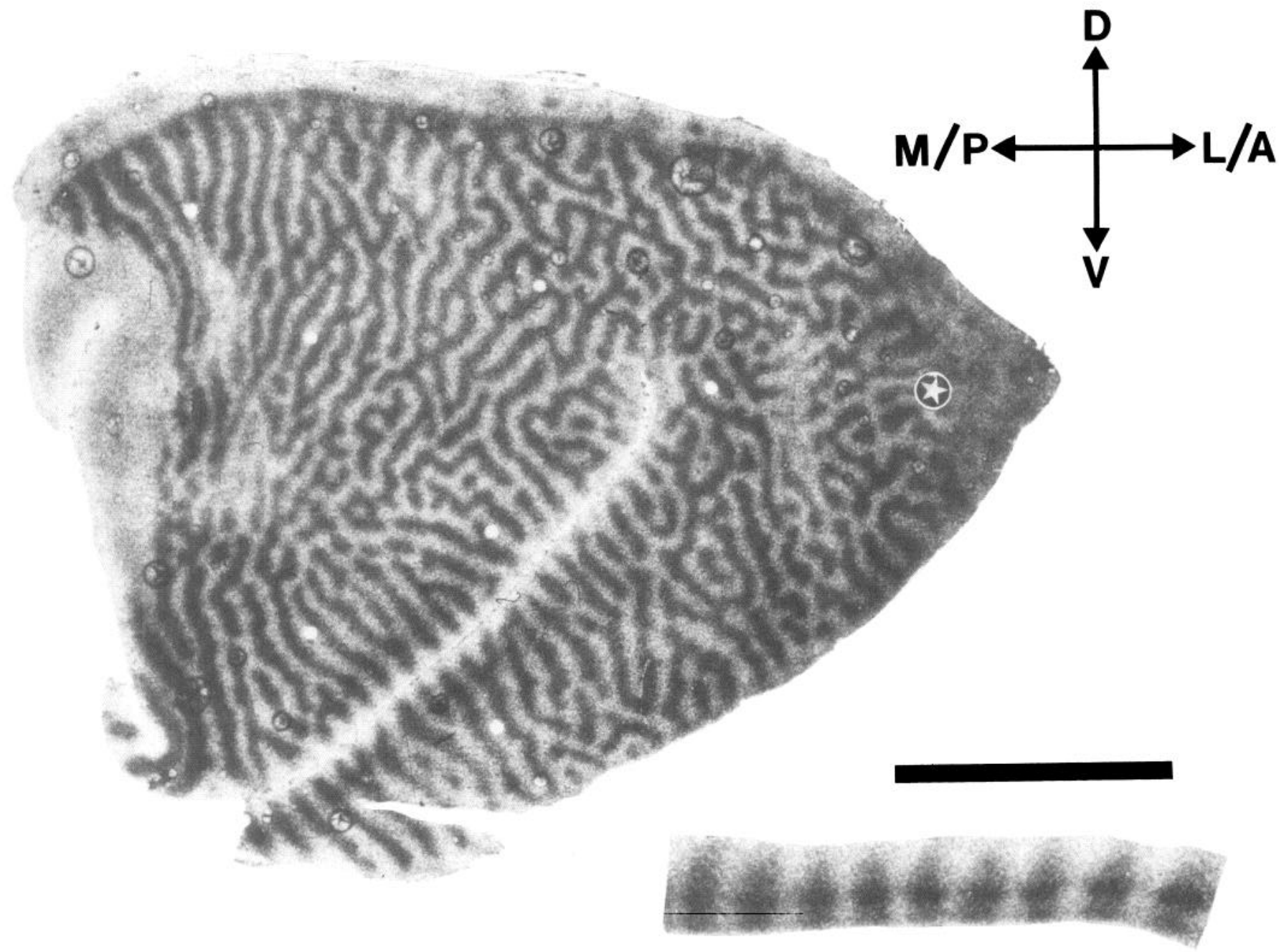

Figure 2. Deoxyglucose autoradiograph showing ocular dominance strips in macaque striate cortex, mostly from layer 3 . The stimulus producing this DG pattern was a moving, black and white square-wave grating of varied spatial frequency, shown at different orientations to one eye. Such a stimulus produces long strips of high DG uptake in all layers that run generally perpendicular to the 17-18 border. The large section shown in this figure was cut parallel with the lateral surface of the unfolded, flat-mounted operculum, and it includes about half the surface area of striate cortex. In this and other illustrations, sections from striate cortex that are unfolded are roughly triangular in shape, with the representation of the fovea at the apex of the triangle (indicated by a star), and the posterior edge of the operculum (representing about $7^{\circ}$ eccentricity) at the base of the triangle along the left-hand side of the picture. The orientation of this section in vivo is roughly indicated by the set of arrows in the upper right. $D$, dorsal, $V$, ventral, $M / P$, medial/posterior, $L / A$, lateral/anterior. Inset, lower right, Laminar distribution of DG uptake in ocular dominance columns in a section cut perpendicular to the cortical surface. The inset is magnified $2 \times$ relative to the large section. Scale bar, $1 \mathrm{~cm}$ relative to large section, $5 \mathrm{~mm}$ relative to inset.

times) were used in each case, the values of DG uptake cannot be directly compared for different cases; thus the values have been normalized in each graph. Values of DG uptake within an animal (e.g., in split-field comparisons within a given layer, or across different layers) can be meaningfully compared. The pattern (as opposed to the levels) of DG uptake could also be directly compared across animals by normalizing exposures with reference to unstimulated regions of striate cortex.

Some stained tissue was photographed through complementary color filters to increase contrast. The original autoradiographs and stained tissue were photographed using Kodak Technical Panchromatic film and Ilfospeed print paper.

\section{Results}

One of the major goals of this study was to investigate how the largely binocular functional activity in visual cortex arises from the monocular inputs to striate cortex. To accurately describe the binocular architecture, we first need to examine the strictly monocular DG architecture, that is, ocular dominance columns.

\section{Ocular dominance columns}

To functionally isolate ocular dominance columns with DG, we occluded one eye of a paralyzed monkey and showed a very general visual stimulus to the other eye. In one representative case (15), the stimulus was a drifting black and white squarewave grating (contrast $>95 \%$ ) of variable spatial frequency ( 1 , 2 , and 4 cycles/deg), shown at multiple orientations (in $45^{\circ}$ steps), moved at a range of temporal frequencies $(2-4 \mathrm{~Hz})$ in a direction that was reversed every $5 \mathrm{sec}$. A white circular aperture limited the stimulus screen to within $6^{\circ}$ eccentricity.

A single autoradiographic section taken from this case is shown in Figure 2. It is taken almost entirely from layer 3, and (except for a few millimeters of tissue wedged into the ectocalcarine fissure) it shows the complete topography of ocular dominance columns within the central $6^{\circ}$ or so. The inset shows a trans- 

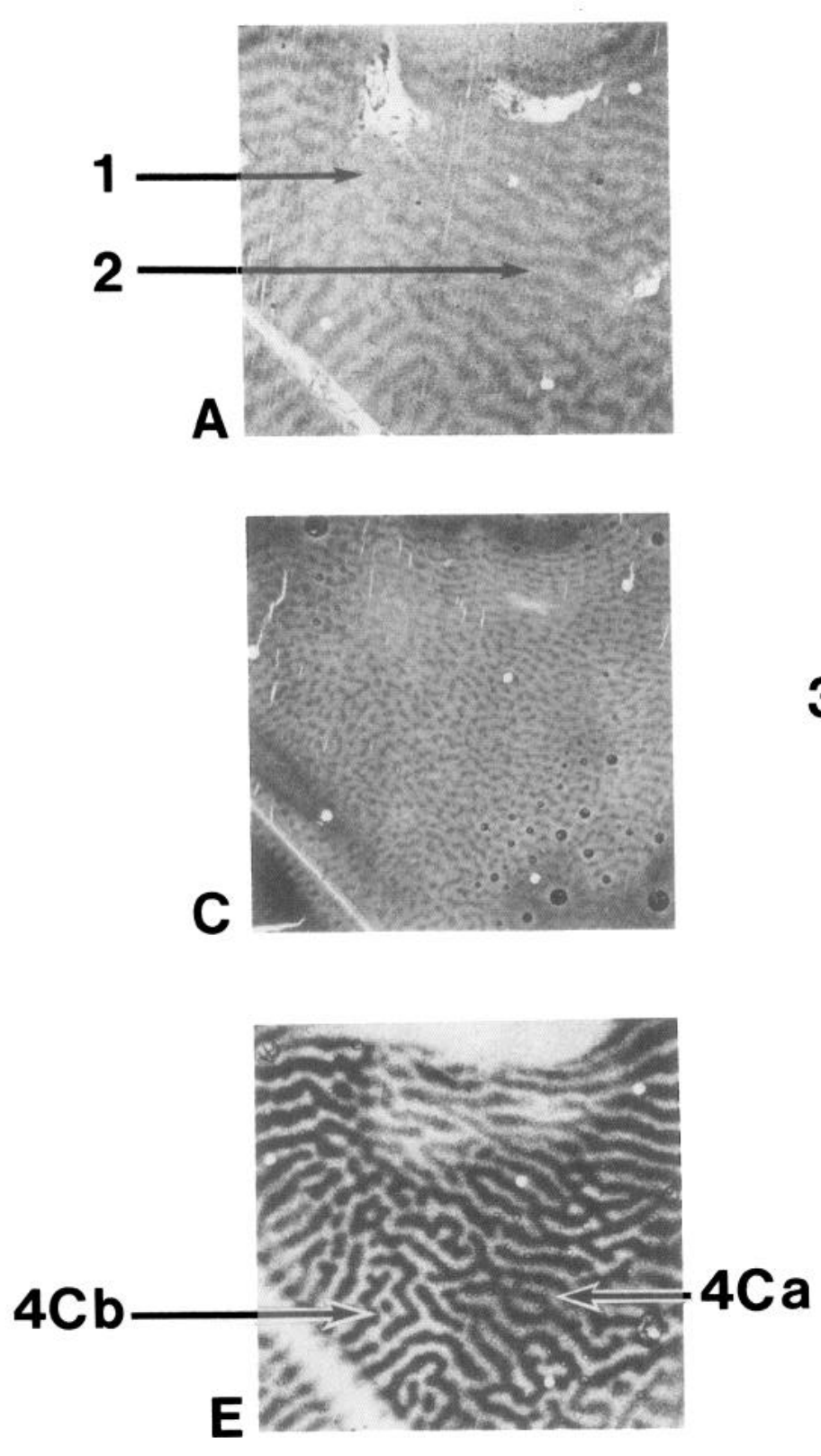
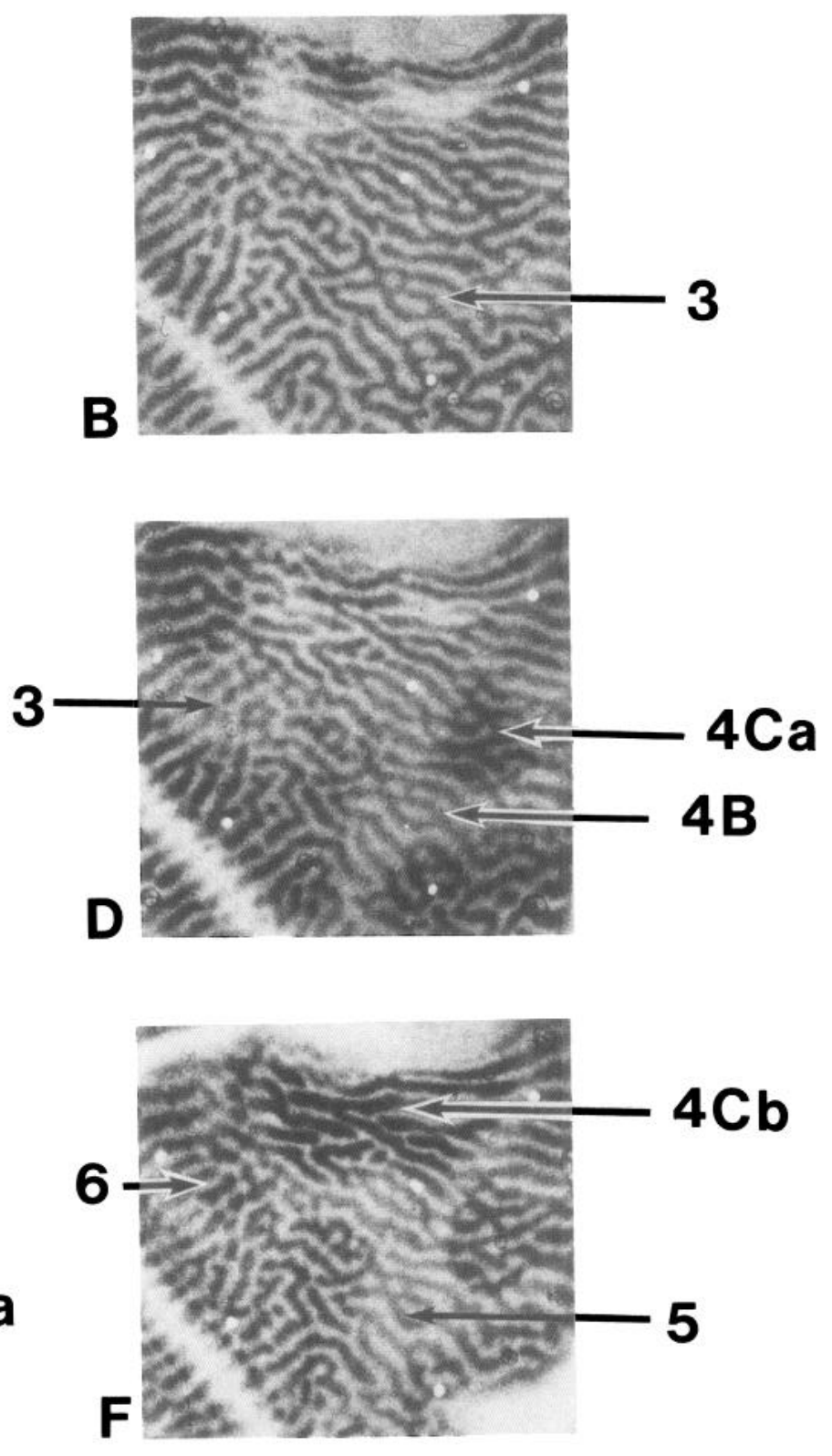

Figure 3. Laminar variations in DG ocular dominance columns. $A, B, D-F$, DG patterns in the same area of cortex from successively deeper sections. The topography of the DG ocular dominance columns remains relatively constant across different layers, although levels of uptake in the stimulated and unstimulated eye dominance columns vary widely. Of special note are the presence of ocular dominance columns in layer $1(A)$ and a significant level of stimulus-induced uptake in the light ("unstimulated") eye dominance columns in $4 \mathrm{Ca}(E)$. A similar pattern of stimulusinduced uptake does not occur in the light ocular dominance strips in layer $4 \mathrm{Cb}$, nor in other layers. $C$, The same section used in $B$, stained for cytochrome oxidase and shown at the same scale. Clearly, the layer 3 ocular dominance strips in $B$ are wider and more uniform in width (that is, more "striplike") than the blobs shown in $C$. Calibration bar, $1 \mathrm{~cm}$.

laminar view of the ocular dominance columns from a deeper section of this hemisphere.

As ocular dominance columns pass through different laminae, a number of changes occur in both the density of the strips and the definition of the strip boundaries. Laminar variations from the case shown in Figure 2 are displayed photographically in Figure 3. As reported earlier, the columns are darkest in layer $4 \mathrm{C}$ and layer 6 , and lighter in layers 2, 3, and 5, 4A, and 4B (Kennedy et al., 1975; Hendrickson and Wilson, 1979). How- ever, there are a number of more subtle laminar variations that are worth describing as well.

With this particular stimulus, density in the dark (stimulated) $4 \mathrm{Ca}$ strips is slightly less than that in the dark $4 \mathrm{Cb}$ strips. However, as we shall see this relationship is dependent on the contrast and color of the stimulus used (e.g., Tootell et al., 1988b, c). The contrast of the strips decreases markedly above layer $4 \mathrm{C}$ and remains relatively constant through layers $4 \mathrm{~B}, 4 \mathrm{~A}$, and 3 . In Figures 2 and 3, layer 4A is indistinguishable from layers 
Figure 4. Densitometric analysis of stimulus-driven DG uptake in the light ("unstimulated") ocular dominance strips. In the case illustrated in Figures 2 and 3, DG uptake was measured in the dark ("stimulated") ocular dominance strips (filled circles), in the light ("unstimulated") ocular dominance strips (unfilled triangles), and in parafoveal regions of the same hemisphere that were visually unstimulated by either eye (filled triangles). Measurements at each point were made by averaging 30 50 values from $25 \times 25 \mu \mathrm{m}$ densitometric samples in each of the indicated layers. Average density measurements were converted to levels of DG uptake by reference to radiographic standards. Except in layer $4 \mathrm{Ca}$, levels of uptake in the light ocular dominance strips are very similar to those in the visually unstimulated regions of the same layer; this indicates that very little uptake is produced in the unstimulated ocular dominance strips by monocular stimulation, except in layer $4 \mathrm{Ca}$. The presence of elevated uptake in the light ocular dominance strips in layer $4 \mathrm{Ca}$ is strong evidence for binocular interactions in this layer.

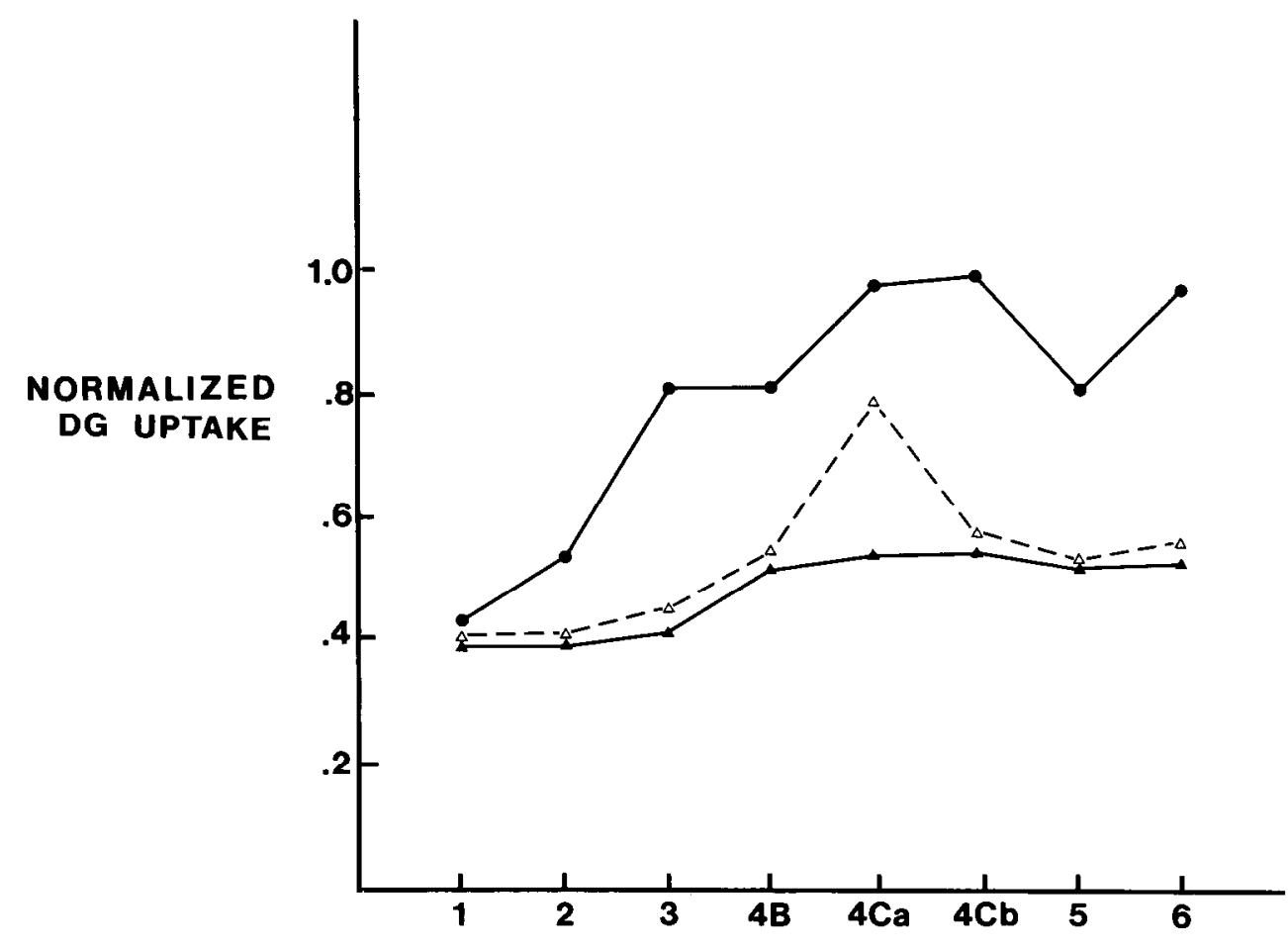

LAYER above and below it, but it can be resolved in other cases shown different visual stimuli (e.g., Tootell et al., 1988b). Above layer 3 , the contrast of the strips decreases gradually. In this and other cases, the DG patterns extend faintly through layer 1 (see Fig. $3 A$ ), which has almost no cell bodies. The presence of DG patterns in layer 1 , though faint, reinforces the presumption that DG is labeling functional activity that is partially nonsomal (Sharp, 1976; Schwartz et al., 1979).

A final point appears to be related to the shape of the topography in different layers. If ocular dominance columns are truly "columnar," the topography of the DG patterns should be identical in all layers, although of course there may be differences in the contrast of the DG patterns across layers. Using this particular stimulus, we in fact find a constant strip- (rather than dot-) shaped topography in all 6 cortical layers (for instance, compare the topography in the various layers shown in Fig. 3).

Using different stimulus conditions, other investigators have reported the topography of ocular dominance columns in the upper and lower striate layers to be dotted instead of striplike (Hendrickson and Wilson, 1979; Horton, 1984). When we stimulate with other, less general, visual stimuli, we also find DG patterns that are bloblike or spotty in the upper and lower layers (see Tootell and Silverman, 1981; Tootell et al., 1982a, 1983, 1985). However, our interpretation of these latter results is that these stimuli label only a subset of the cell processes related to one eye in the extragranular layers, i.e., with certain stimuli one does not label the full ocular dominance "column." It is easier to imagine ways in which a truly columnar (striplike) eye dominance structure could appear artifactually "dotlike" in the extragranular layers than it is to imagine the converse. These results really bear on the question of baseline DG uptake and cytochrome oxidase activity, which is discussed more fully below.
Since we were especially interested in binocular interactions within striate cortex, it seemed sensible to first see how "monocular" the ocular dominance columns truly were. Specifically, in layers that are to some extent binocular, one might expect a higher level of DG in the light ocular dominance strips relative to that found in unstimulated areas of striate cortex within the same layer. To resolve this issuc, densitometric measurements of DG uptake were made in (1) the dark strips, (2) the light strips, and (3) visually unstimulated areas of striate cortex, from each layer of the case illustrated in Figures 2 and 3.

These data are shown in Figure 4. Given the number of striate cells showing binocular interactions (Hubel and Wiesel, 1977; Poggio and Fischer, 1977), it is surprising that only layer $4 \mathrm{Ca}$ shows elevated ("binocular") uptake in the light ocular dominance strips. This stimulus-driven elevation of uptake in the "light" ocular dominance strips of $4 \mathrm{Ca}$ can also be seen clearly in the autoradiographs themselves (see Fig. $3 E$ ). It is particularly intriguing that such a robust binocular DG enhancement should appear in a striate geniculorecipient layer, and not in subscquent striate layers that have been characterized as being more binocular by electrophysiological studies. This DG evidence for interactions across ocular dominance strips is the first of several pieces of evidence for binocular interactions in magnorecipient layer $4 \mathrm{Ca}$, which do not appear in parvorecipient layer $4 \mathrm{Cb}$ (see below).

On the basis of several kinds of evidence, it has been reported that cytochrome oxidase blobs lie in the center of ocular dominance columns (Hendrickson et al., 1981; Horton and Hubel, 1981; Horton, 1984). This DG-cytox relationship is almost a logical necessity because alternate rows of cytox blobs blanch following removal of one eye (Horton and Hubel, 1981; Horton, 1984). Nevertheless, since our own DG ocular dominance results are "striplike" rather than "bloblike" in layer 3 , and since 

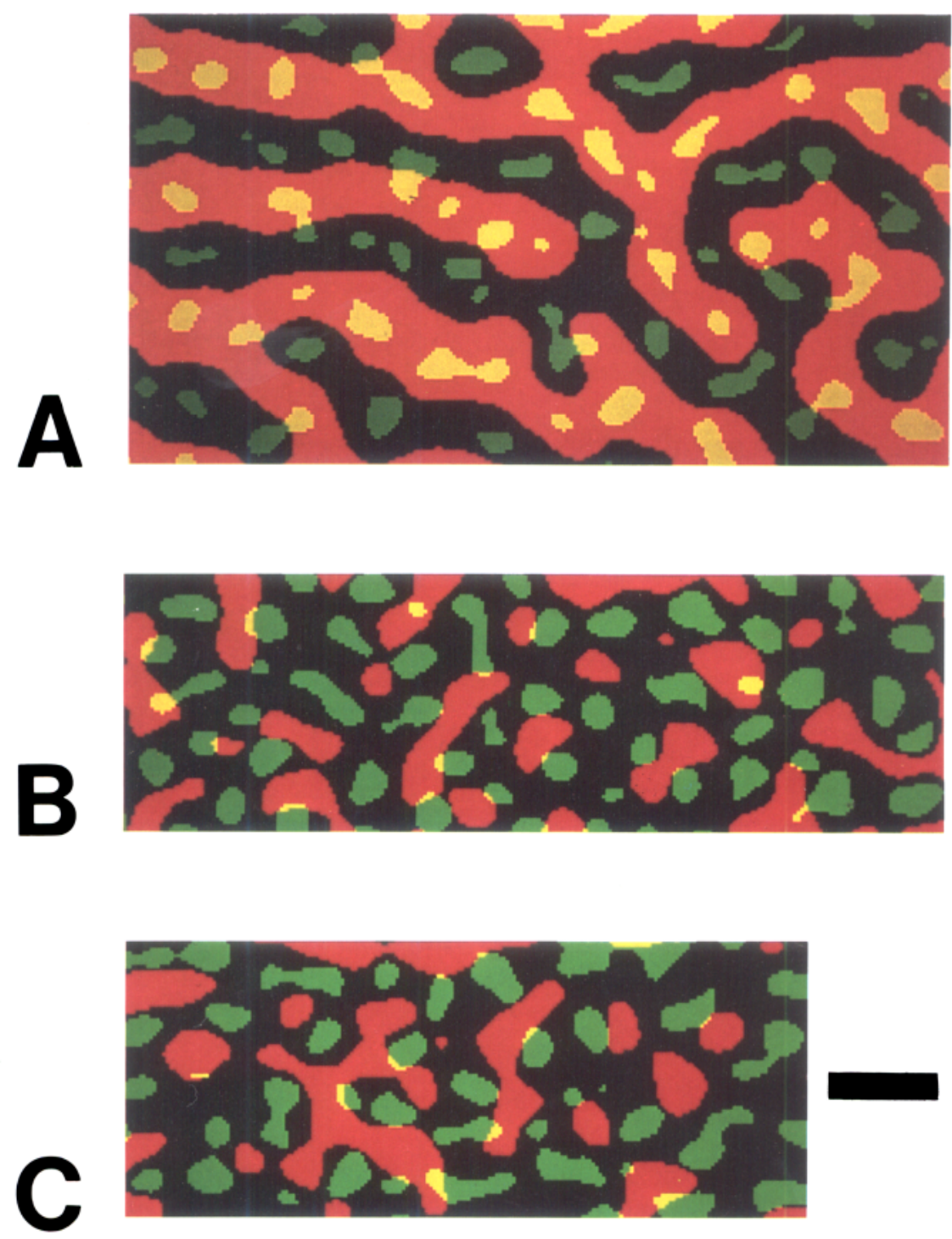

Figure 5. Densitometric analysis of the topographic relationship between cytochrome oxidase activity and DG uptake in response to 2 different visual stimuli. In $A-C$, measurements are made within a single section taken from layer 3 of macaque striate cortex. Regions of cytochrome oxidase activity above a certain densitometric level are color-coded green, and regions of high DG uptake are color-coded red. Regions of low DG uptake (and low cytochrome oxidase activity) are colorcoded black. Cortical regions where there is both high cytochrome oxidase activity (green) and high DG uptake (red) appear yellow. For further details, see Switkes et al. (1986). In $A$, the animal was stimulated monocularly with a pattern of black and white stripes of different widths, shown at different orientations, moved at different speeds in different directions. At parafoveal eccentricities, the resulting ocular dominance columns are striplike rather than blob-shaped, covering approximately half the surface area of striate cortex in all layers, including layer 3 . As noted previously, the cytochrome oxidase blobs lie in the center of the ocular dominance strips. $B$ and $C$ are taken from an unanesthetized, unparalyzed animal that was shown (binocularly) a high-spatial-frequency ( 7 cycles/deg) grating that was systematically varied in orientation. $B$, The topography of DG uptake at an eccentricity near $5^{\circ}$. $C$, The pattern of DG uptake near $8^{\circ}$ along the underside of the operculum. It has been previously reported that in unanesthetized and unparalyzed animals shown stimuli of low- or mixedspatial-frequency components, DG uptake is highest in the blobs (Kennedy et al., 1975; Humphrey and Hendrickson, 1983). However, following stimulation with a high-spatial-frequency grating $(B$, $C)$, DG uptake is clearly highest between the blobs, rather than in the blobs themselves. Calibration bar, $1 \mathrm{~mm}$. the ocular dominance-cytox relationship has not been examined within a single section, it seemed worthwhile to look at this relationship in more detail.

We analyzed the DG-cytox topography in the case illustrated in Figures 2-4; the results of the present analysis are shown diagrammatically in Figure 5. It is clear from Figure 5 that the cytochrome oxidase blobs do indeed lie in the centers of the ocular dominance strips. It is also clear that the ocular dominance strips are quantitatively striplike rather than dotted; even if we isolate the regions of very high DG uptake, these regions do not always overlie the cytox blobs (not illustrated).

It has been reported a number of times (by ourselves as well as others) that the cytox blobs in the upper layers overlie the light cytox blobs in the lower layers. However, we are not aware of any systematic study of the correspondence (or lack thereof) between upper-layer and lower-layer cytox blobs. In order to resolve this matter, we used the ocular dominance columns to help align the upper- and lower-layer blobs. First, the ocular dominance strip topography in sections from layer 3 and layer 5 were carefully aligned (see Fig. $6, A, C$ ). By using cytox-staining patterns from the same section, it then became possible to align the obvious layer 3 cytochrome oxidase blob array with the very faint layer 5 blob array (see Fig. 6, $B$ and $D$ ). From this and other comparisons, it appears that the upper and lower cytochrome oxidase blobs are indeed in vertical registration.

Our primary interest in this study was not the topography of ocular dominance strips per se. However, in the course of doing monocular experiments we were able to examine the topography in a large number of animals, and several points are worth noting (see Fig. 7).

First, there is a great deal of variation between the overall topography of hemispheres in the same animal: the DG topography in each hemisphere is clearly not mirror-symmetric (compare Fig. 7, $A$ and $B, C$ and $D$, and $E$ and $F$; cases 15, 23, and 22 , respectively). Thus, the ipsilateral set of ocular dominance strips is not identical to the contralateral set. It remains remotely 


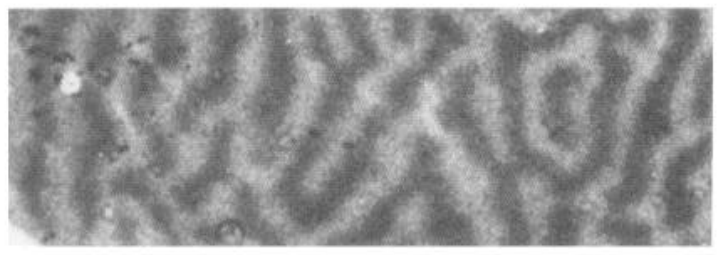

A

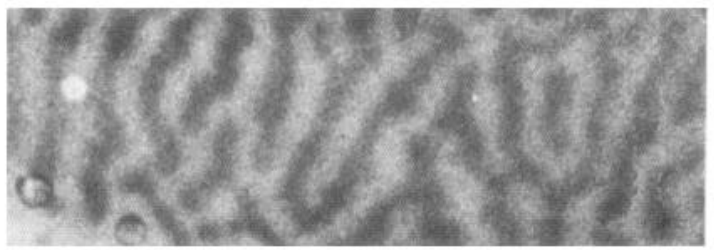

C

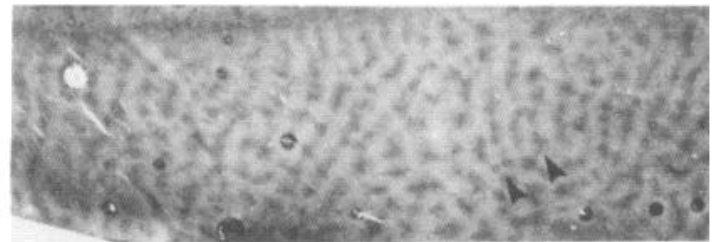

B

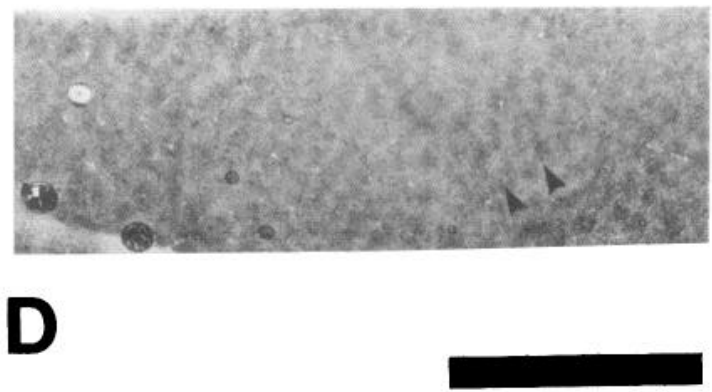

Figure 6. Demonstration that the lower-layer blobs underlie the upper-layer blobs, using ocular dominance columns as aids to alignment. $A$, $C$, A portion of the DG ocular dominance topography from the case shown in Figure 2. $A$ is taken mostly from layer 3 and $C$ from layer 5 . Corresponding regions from the 2 layers are vertically aligned. $B, D$, Cytochrome oxidase staining patterns from the sections used in $A$ and $C$, vertically aligned as in $A$ and $C$. By carefully examining the topographies in $B$ and $D$, one can confirm that the cytochrome oxidase mottling in layer 5 corresponds to the higher-contrast blob pattern in layer 3 (see arrow). Calibration bar, $5 \mathrm{~mm}$.

possible that the ipsilateral set of ocular dominance strips bears some resemblance to the contralateral set in the other hemisphere, but this is certainly not obvious from the data in Figure 7.

Second, there is a certain species-specificity to the overall topography. For instance, Figure 7, $E$ and $F$, are taken from $M$. fascicularis, and show a characteristic "funneling" of ocular dominance strips perpendicular to the posteromedial edge of the operculum. A topographically similar pattern was described earlier in either a $M$. fascicularis or M. mulatta (LeVay et al., 1975 ) or in a M. fascicularis (LeVay et al., 1985). However, in other species, such as $M$. arctoides, this funneling is either drastically reduced or absent (e.g., Fig. 7, $A-D$, and unpublished observations). Other aspects of the previously reported topography are more generalizable across different species: there is a strong tendency for ocular dominance strips to run perpendicular to the V1-V2 border in all macaque species examined. This is not particularly surprising, since it also occurs in man (Horton and Hedley-Whyte, 1984).

Third, there is a variation in the absolute width of ocular dominance strips in different species: species with large bodies (and large brains) have wider ocular dominance columns, as well as larger distances between adjacent cytox blobs. At eccentricities of $2^{\circ}-5^{\circ}$, we found an average spacing (for a complete set of ocular dominance strips) of $980 \mu \mathrm{m}$ in $M$. arctoides, 730 $\mu \mathrm{m}$ in $M$. assamensis, $650 \mu \mathrm{m}$ in $M$. radiata, and $680 \mu \mathrm{m}$ in $M$. fascicularis. The spacing between blobs is correspondingly greater in $M$. arctoides than in $M$. fascicularis, primarily in the dimension perpendicular to the long axis of the ocular dominance strips (see Tootell et al., 1988a). There is a parallel increase in the size of ocular dominance strips and cytox blobs in higher primates: the human brain and the between-blob spacing in the human are larger still (Horton and Hedley-Whyte, 1984; see also Tootell et al., 1988a). Because of this interspecies variation in striate blob spacing, we generally describe topographic variation in terms of a geometric relationship to the cytox blobs in the same tissue section, rather than as measurements of absolute intercolumnar distance.

\section{Binocular stimulation}

In our hands, monocular stimulation with a very general stimulus produced DG ocular dominance strips that covered about half of the surface area in all layers of striate cortex. We therefore assumed that binocular stimulation with the same visual stimulus would produce a pattern of DG uptake that was topographically rather uniform in all layers.

This was tested in 4 different animals (cases 24, 37, 42, 50); results from all 4 animals were quite similar. In one representative case (37), the stimulus was quite similar to that used to produce the ocular dominance topography in Figure 2, except that the monkey viewed the stimulus with both eyes open instead of through one eye. Again, the stimulus was a black and white square-wave grating shown at various orientations, spatial frequencies, temporal frequencies, directions, etc. In this particular case, we kept binocular disparity constant, but in other cases the binocular disparity was systematically varied. (Variable- and constant-disparity conditions produced DG results that were indistinguishable-see below.) In this case, the stimulated portion of the visual field extended only to $4.6^{\circ}$ on both sides of the midline, along a vertical (rather than a circular) border.

The resultant layer $3 \mathrm{DG}$ pattern is shown across the whole operculum in Figure $8, A, C$. This figure shows (from fovea towards the periphery) a large region stimulated binocularly ("B" in Fig. 8, $A, C$ ), a small monocular segment due to minor 

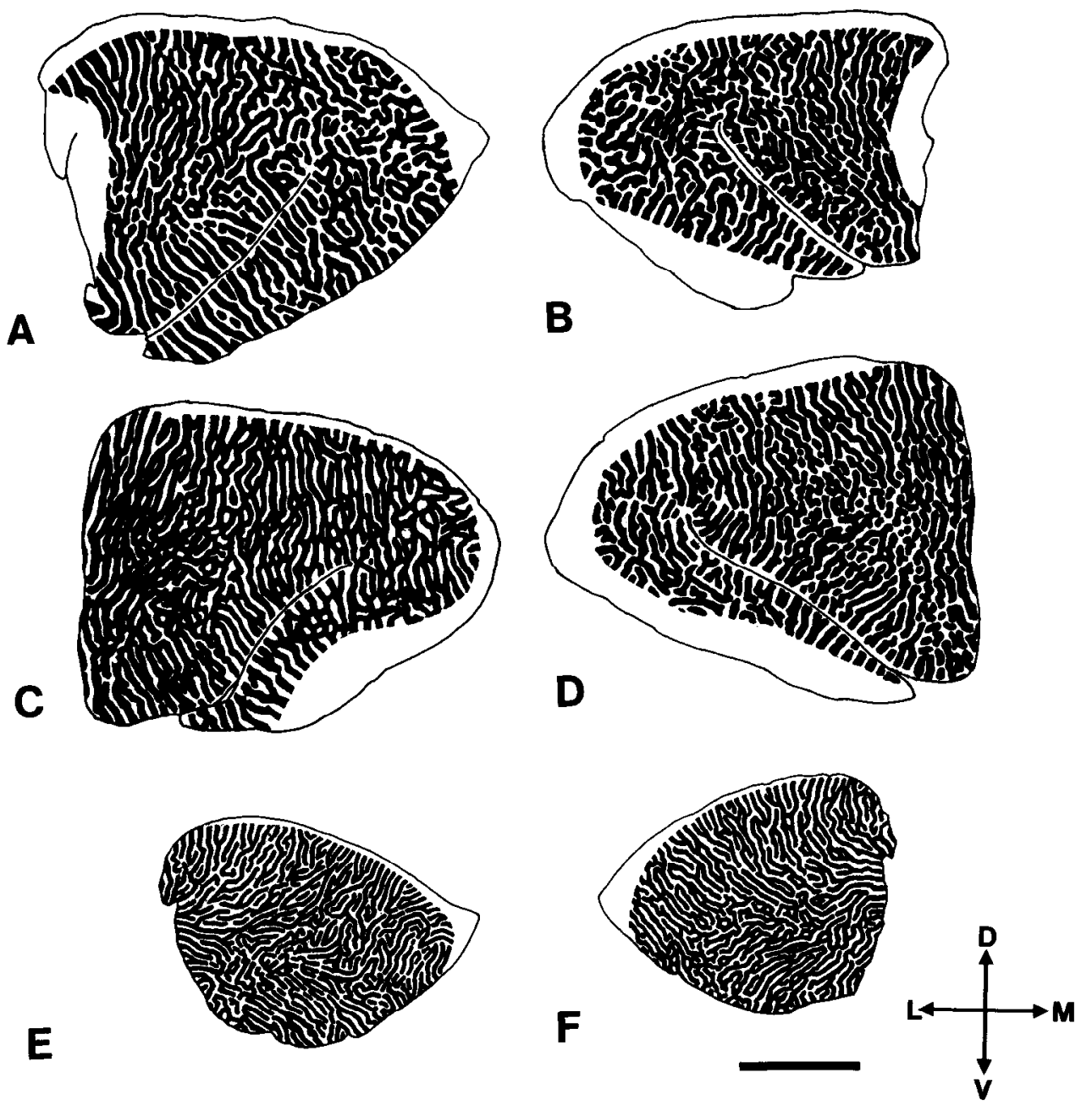

Figure 7. Overall topography of ocular dominance strips across the lateral operculum in 3 different animals of 2 macaque species. The topography was drawn from the layer 4 DG pattern produced by stimuli, as described in Figure 2. Paired hemispheres $(A$ and $B, C$ and $D, E$ and $F$ ) are taken from the same animal. The representation of the fovea (lateral in vivo) appears towards the middle of the figure (far right in $A, C$, and $E$, and far left in $B, D$, and $F$ ). The representation of the periphery (medial in vivo) appears to the far left in $A, C$, and $E$, and to the far right in $B, D$, and $F$. Dorsal in vivo is towards the top of each section, and ventral in vivo appears towards the bottom of the page. For $B, D$, and $F$, these relationships are indicated by the compass-shaped symbol to the right of $F$. The topography in the top 2 animals $(A-D)$ is taken from $M$. arctoides, and the patterns in $E$ and $F$ are taken from $M$. fascicularis. These topographic patterns are representative of those seen in other cases. In the fascicularis $(E, F)$, the ocular dominance strips show a characteristic "funneling" parallel with the horizontal meridian near the posterior medial border of the operculum (extreme left and right sides of $E$ and $F$, respectively). No such funneling appears in $M$. arctoides. The dominance strips are significantly wider in $M$. arctoides than in $M$. fascicularis. This appears to be correlated with a larger brain size (and a larger striate area) in $M$. arctoides relative to $M$. fascicularis. In some animals (of each species), there appears to be a contralateral-ipsilateral difference in the surface area (and topography) of ocular dominance strips between hemispheres (e.g., $C$ vs $D)$. Scale bar, $1 \mathrm{~cm}$. misalignment of the eyes ("M"), and portions of the same layer that viewed adjacent screen regions set at a gray of the same luminance (" $U$ ") as the mean of the stimulus luminance. The cytox pattern from the same sections is shown in Figure 8, $B, D$.

A closeup of the DG pattern in Figure $8 A$ is shown in Figure 9, top. The DG pattern in Figure 9, top, is taken from the same eccentricity $\left(2^{\circ}-5^{\circ}\right)$ as the monocular DG result shown in Figure 3. At this parafoveal eccentricity (where we have done most of our DG-cytox analysis), both the photographs and the original autoradiograph produced by this binocular stimulus look relatively uniform within the blob-dominated layers.

It is conceivable that nonlinearities in the original $x$-ray film could have produced an artifactually flattened DG pattern. Though this seems unlikely, we controlled for this possibility by converting the autoradiographic optical densities to DG uptake via standards, and by sampling uptake levels across stimulated and unstimulated parts of the autoradiograph (see Fig. 9 , bottom). The data points presented correspond to the average of densities of a sampled point and its nearest neighbor. Smoothing between data points was minimal in this case, so as to avoid averaging out any blob biases in the patterns of uptake. As can be seen in Figure 9, levels of uptake show no obvious blob bias in uptake, although there is some drift in absolute level due to drift in laminar depth.

Nearer the fovea, the stimulus described above sometimes produces slightly more DG uptake in the cytox blobs than in the interblobs (see Fig. 8). We refer to this general finding as a "foveal blob bias." The foveal blob bias appears to be due largely to a decrease in interblob uptake near the fovea, relative to both the foveal blobs and to more peripheral blob and interblob regions. A fainter blob bias in uptake also appears near the fovea in other layers that have fainter cytox blobs (e.g., layer 2 and very faintly in layer 6).

On the basis of evidence from related experiments using sinusoidal gratings (see Tootell et al., 1988d), we presume that this blob bias is due to an upward shift in the average spatial frequency tuning (associated with smaller receptive-field sizes) of cells in the foveal region, relative to the constant (and relatively low) frequency range of the stimulus pattern. The foveal blob bias may also be exaggerated by the binocular stimulation conditions (see below). However, a similar foveal blob bias in DG uptake can be seen in many of the monocular cases. In monocular cases where it is less obvious (e.g., Fig. 2), we presume this to be due to perceptual masking caused by the predominant ocular dominance architecture. 


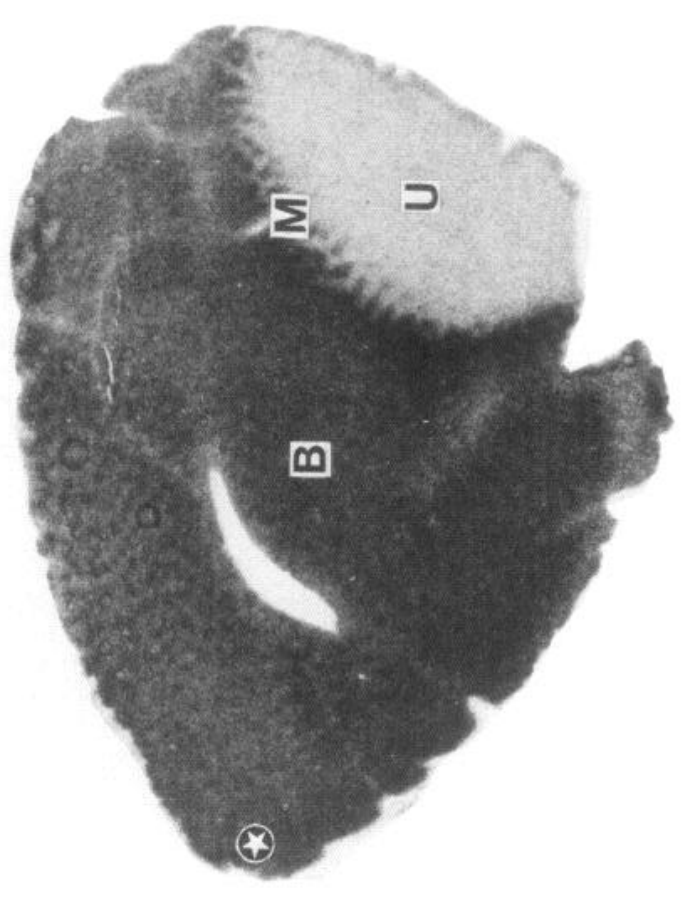

U

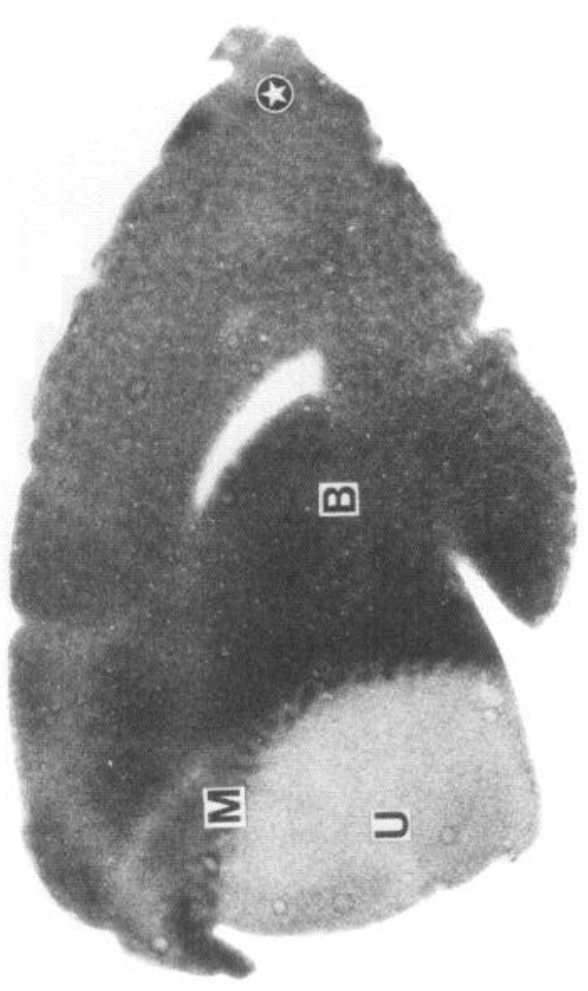

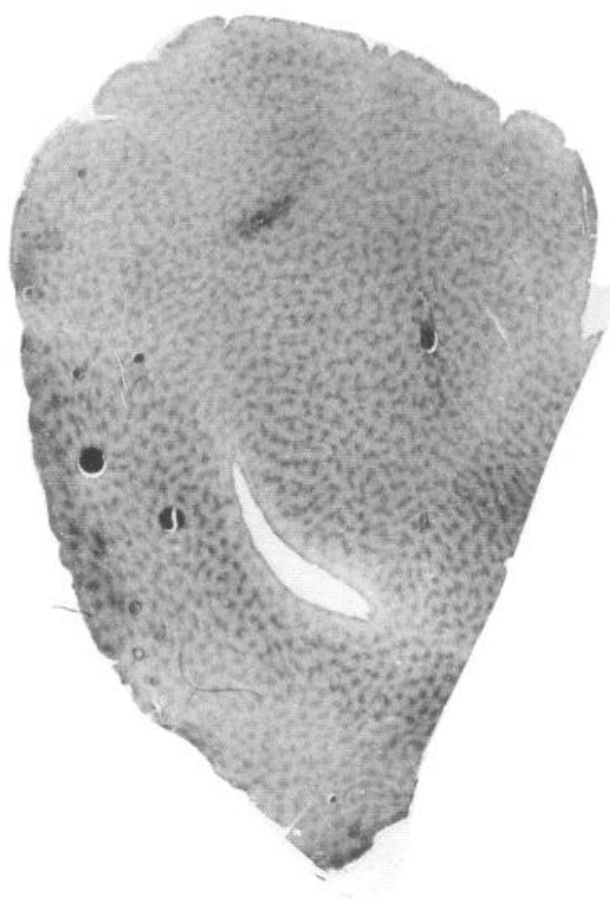

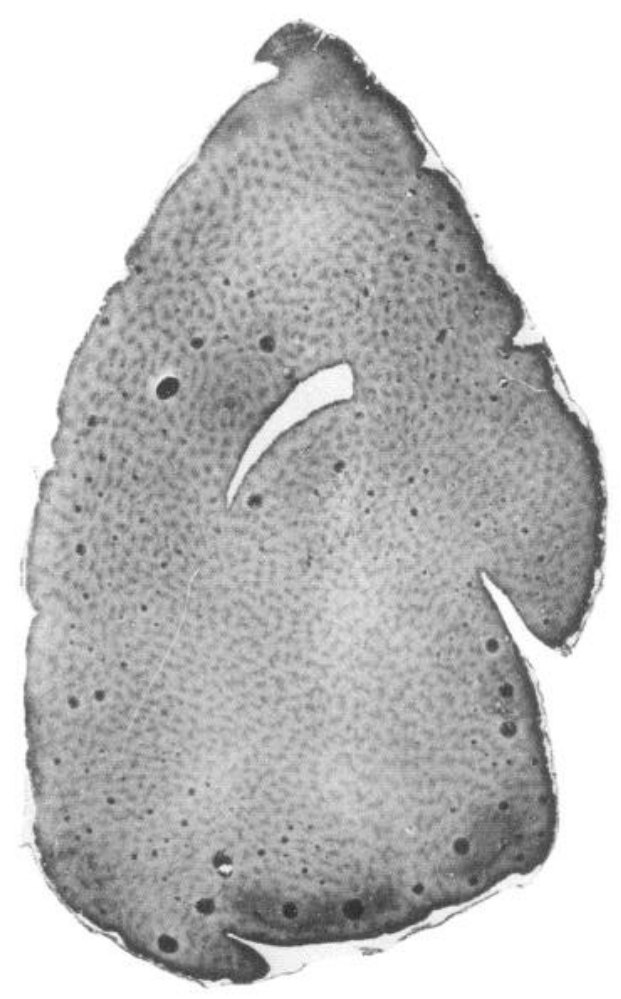

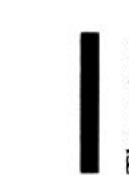



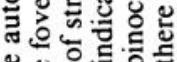

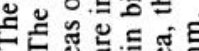

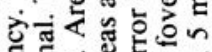

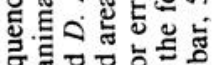

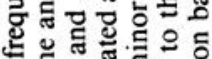
닐 政

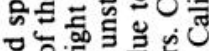

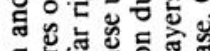
을 刃.

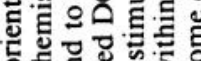
동

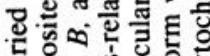
路을

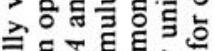

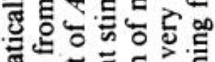

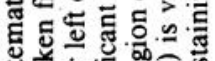
产急的的 ลิ

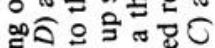

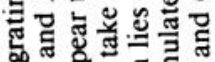

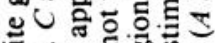
os

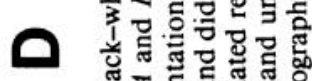

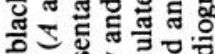
o

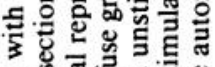

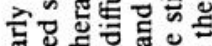
蛋 웡등 당 设

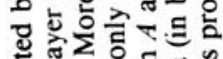

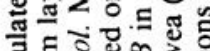

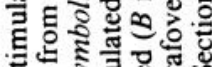

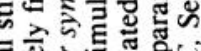

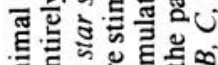

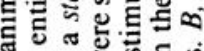

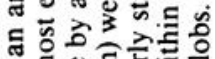

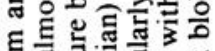

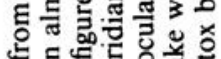

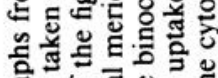

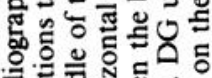

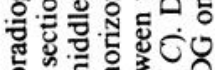
近品

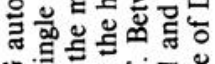

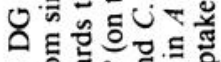
ن온을

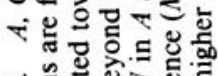

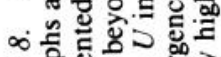
규워 5.

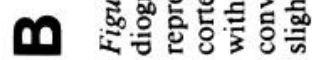




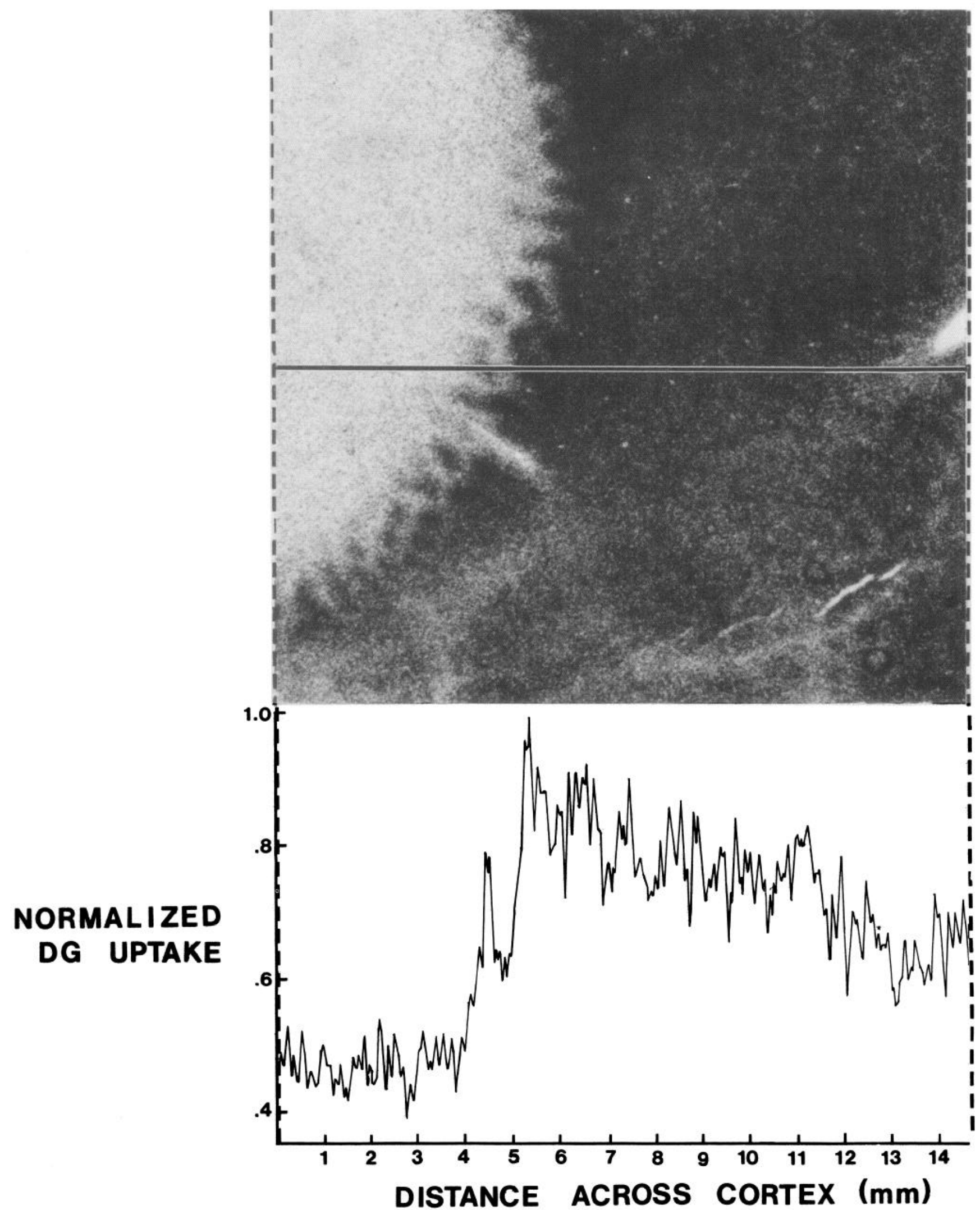

Figure 9. Densitometric analysis of the topography of uptake in layers $2+3$ in the parafoveal representation of the section illustrated in the right-hand portion of Figure $8 \mathrm{C}$. The animal in this case was stimulated binocularly with an achromatic square-wave grating of systematically varied orientation and spatial frequency. The original autoradiograph is shown at the top. From left to right along the horizontal line in the top section, densities were sampled across the unstimulated region, the small monocular segment, and the binocularly stimulated representations of layer 3. Densities were sampled through a $25 \times 25 \mu \mathrm{m}$ aperture, converted to levels of radioactivity by using radioactive standards, and plotted at the same scale for the bottom graph. It is clear that the relative uniformity of the topography in the binocular segment is not due to saturation of the photographic emulsion; rather, it is due to a relative uniformity of uptake in the parafoveal representation. The shift in overall level of DG uptake from left to right of the binocularly stimulated area is due to an enhancement of DG activity at the stimulus border, coupled with a slight laminar shift upwards to the right of the scanned region. 

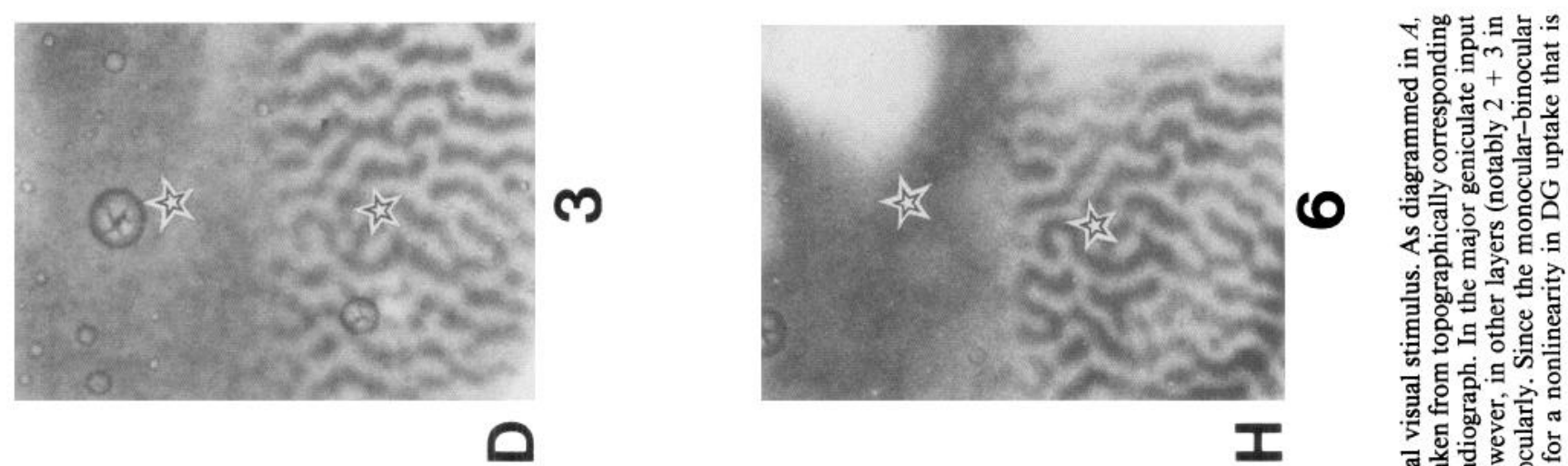

(1) अँच

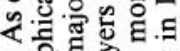

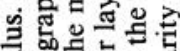

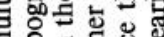
自递和部. कह 젹 5.

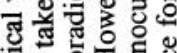

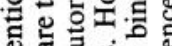
政安. 的矛到
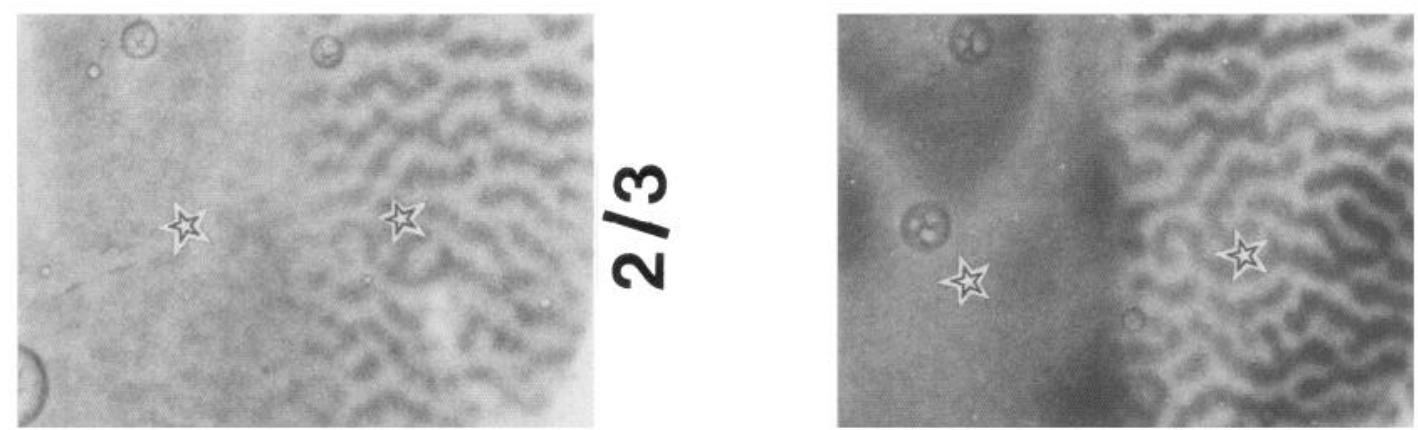

的记

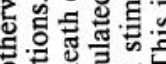
두의.

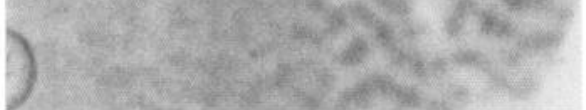

0
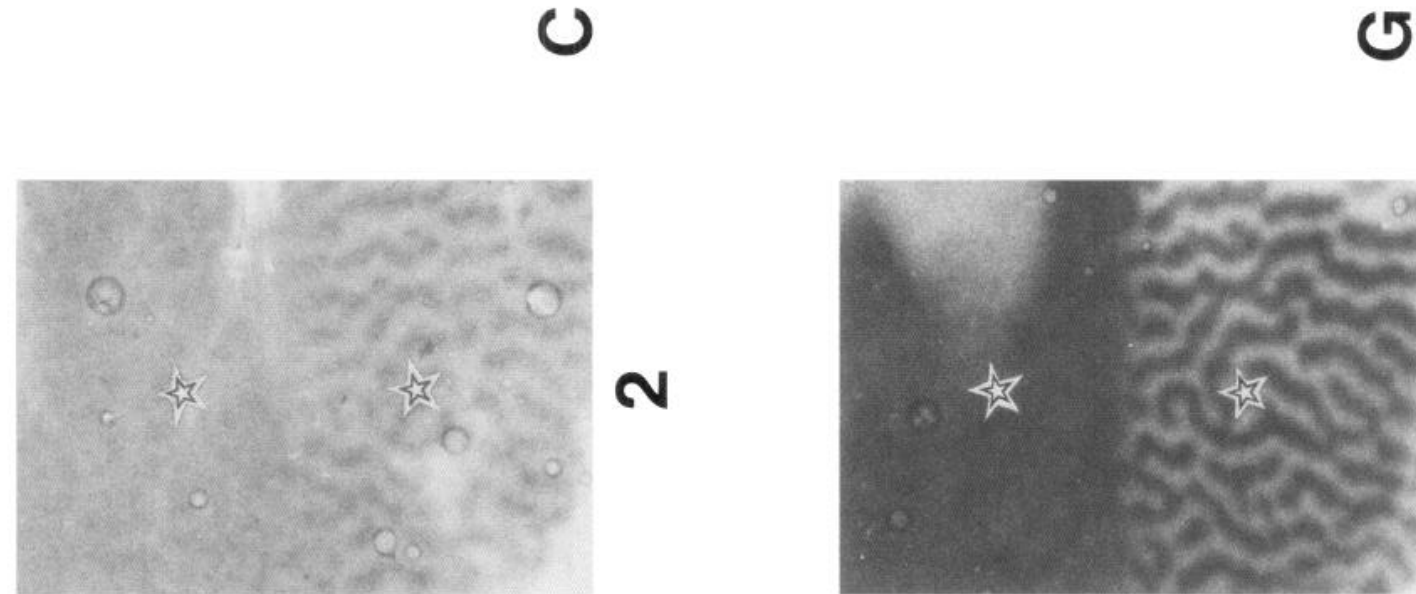

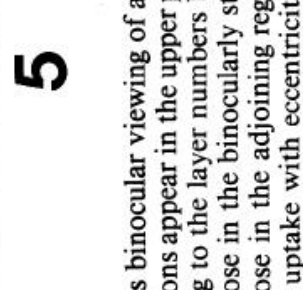

$\checkmark$

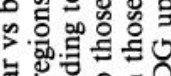

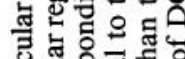

要

\%. 흘

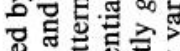

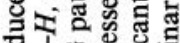
인

$\boldsymbol{m}$

ᄂ
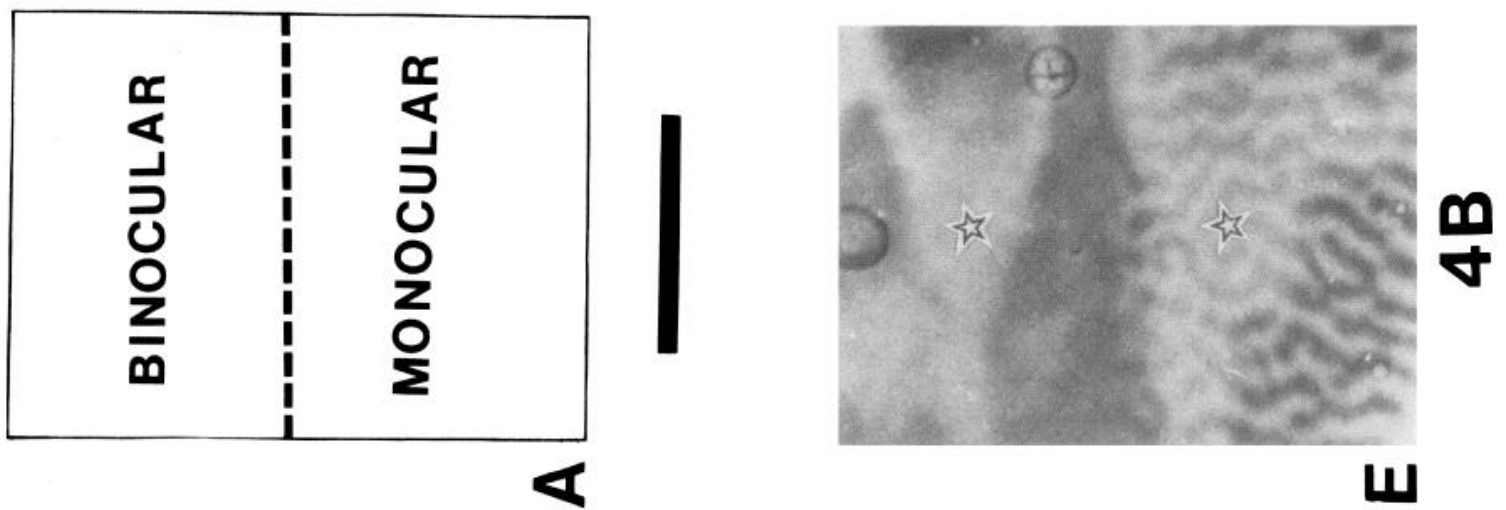

(1) ) $\checkmark$ 을 을 它. 造要 흐의의 웡 क

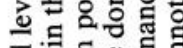

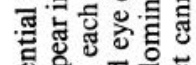

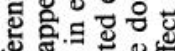

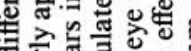

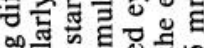

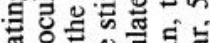
등 흐을 정

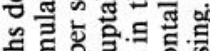
2.

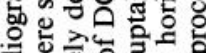
낸

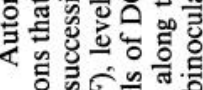

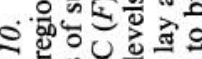

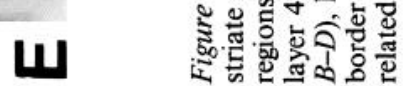




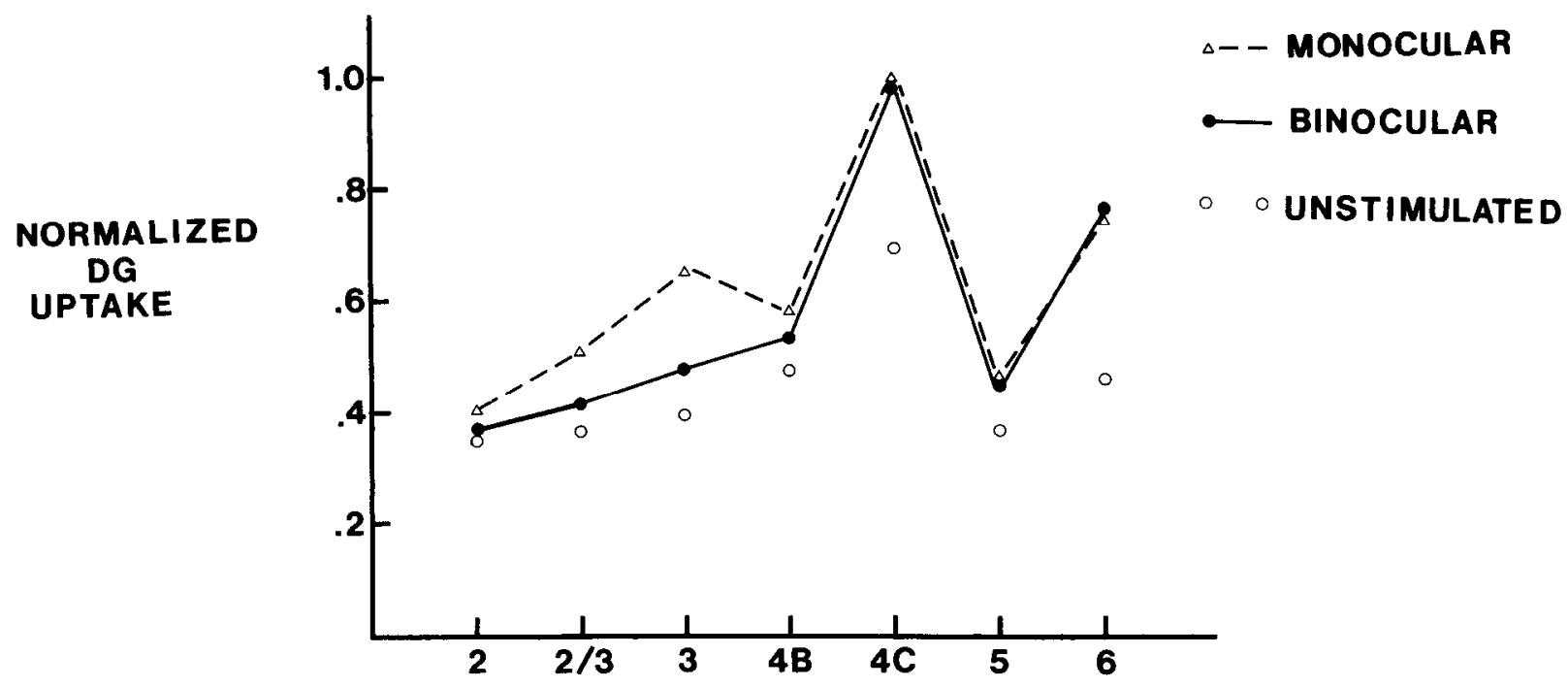

\section{LAYER}

Figure 11. Densitometric comparison of DG uptake during conditions of monocular vs binocular visual stimulation. In Figure 10, densitometric measurements were made in the binocular region (filled circles), the dark ("stimulated") ocular dominance strips (unflled triangles), and the light ("unstimulated") ocular dominance strips (unfilled circles). Measurements in each layer were made by averaging $30-50$ values from $25 \times 25 \mu \mathrm{m}$ densitometric samples, and then converting those average density values to DG uptake via radiographic standards from the same piece of film. In layers $4 \mathrm{C}, 5$, and 6 , levels of uptake in the stimulated ocular dominance strips are essentially equal to those in the adjacent binocularly stimulated region; this indicates a lack of binocular interaction in the DG results within these layers. In layers 2 and 3 (and perhaps 4B), levels of uptake in the binocularly stimulated region are less than those in the corresponding monocularly stimulated regions of the same layer. This indicates the presence of binocular interaction in the DG results from layers 2 and 3, and perhaps from 1 and $4 \mathrm{~B}$.

Alternatively, it is conceivable that the decreased uptake in interblob regions towards the fovea could indicate a real difference in the metabolic architecture near the fovea, perhaps related to that for binocular vision. However, if the metabolic architecture of the cytox blobs and interblob areas is different near the fovea, one might very well expect a parallel decrease in the average levels of cytochrome oxidase staining in the foveal interblob areas, and this does not occur. A foveal blob bias shows up in other experimental contexts, which are examined in greater detail below.

\section{Binocular interactions}

In these binocular experiments, it seemed possible that the fixed angle of eye convergence might lead to artifactual DG patterns due to constant stimulation at zero disparity or at a disparity other than zero. We tried to control for this possibility in 5 animals (cases 19, 20,28, 32, 37) by varying the binocular disparity continuously over a certain small range while the animal viewed the DG stimulus (see Materials and Methods). Within the binocularly stimulated region of these animals, DG results were indistinguishable from those in cases in which animals were shown identical visual stimuli while binocular disparity was left constant during DG infusion.

In cases where binocular convergence was adjusted in an attempt to match zero disparity, and then left constant during DG infusion, the actual binocular disparity would sometimes turn out to be significantly different from zero. The presence and magnitude of any true convergence error was obvious from the presence and size of a monocular segment at the stimulus borders in the resultant autoradiographs (see Fig. 9, top). Except at the stimulus borders, results from cases in which disparity was slightly off-zero did not differ in any obvious way from zero-disparity cases. For instance, the pattern of DG uptake in case 37 (where binocular disparity was varied continuously during the period of active uptake) is not obviously different from that illustrated in Figures 8 and 9. Figures 8 and 9 were taken from an animal shown an identical stimulus, except that the disparity was kept constant, and in this case there was a minor misconvergence of the eyes. Furthermore, the patterns in both of these cases do not differ obviously from the pattern in case 42 , where the eyes were exactly converged.

These results are comforting in one sense because they obviate the need for extensive disparity-control manipulations in other experiments using binocular visual stimuli. In another sense, however, they are disappointing because they offer no evidence for a topographically organized striate architecture concerned with binocular disparity processing, such as that described by Blakemore (1970) in the cat or Clarke et al. (1976) in the sheep. In fact, however, there are several DG results that $d o$ appear to be related to binocular disparity.

So far, we have discussed aspects of DG patterns in which the binocular result is approximately the lincar sum of duplicated monocular DG patterns. For instance, stimuli that produce high DG uptake on half of the cytox blobs when viewed monocularly will produce high DG uptake on all of the cytox blobs when viewed binocularly. Stimuli that produce uniformly flat ocular dominance strips in the parafovea when viewed monocularly (Fig. 3) produce a uniform parafoveal uptake when viewed binocularly (Fig. 9). However, in several other respects the binocular DG patterns deviate from a linear summation of monocular patterns. As we mentioned before, these deviations are extremely important to analyze because they may give us a clue about the architecture of binocular processing.

First, in comparisons between animals, the pattern of uptake 


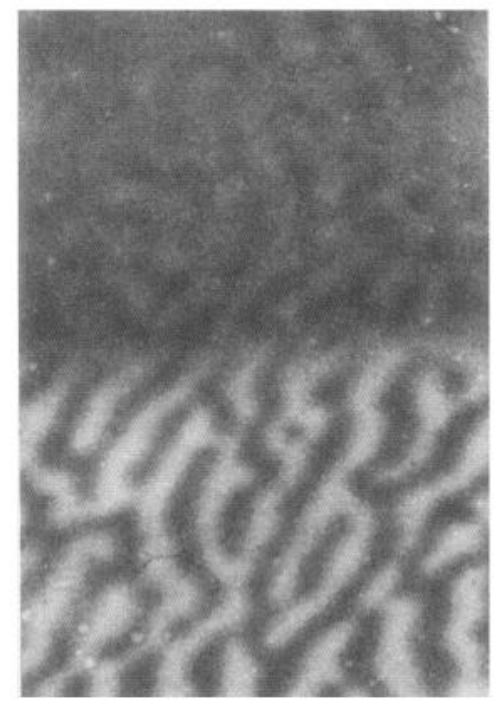

Figure 12. Layer $4 \mathrm{Cb}$ autoradiograph from a comparison of monocular and binocular viewing conditions similar to that described in Figure 10. However, in this case the "binocular" segment (upper half) showed some low-contrast "semimonocular" dominance strips within layer $4 \mathrm{Cb}$. These appear to be due to the presence of slight optical problems in one of the 2 open eyes. In the other layers (not shown), no such inhomogeneities could be seen within the binocular segment. Calibration bar, $2 \mathrm{~mm}$.

seemed to differ across laminae less in monocular cases than in binocular cases. In order to be certain that this effect was genuine, we arranged a very general stimulus so that both the monocular and the binocular border enhancement of the same stimulus could be examined in the same hemisphere (thus, from a single animal), at the same eccentricity (case 50 ). This was done simply by inserting a thin piece of white mounting cardboard in front of one eye, above and bordering the horizontal meridian. Thus, striate regions on one side of the horizontal meridian were stimulated binocularly, and those on the other side were stimulated monocularly. In this case the binocular disparity was not varied. The stimulus in this case was a blackwhite square-wave grating of either $1.4,2.4$, or $3.4 \mathrm{cycles} / \mathrm{deg}$, shown at all orientations (in $45^{\circ}$ steps), moved across the screen in alternating directions.

A parafoveal border region from several layers of this case is shown in Figure 10. Density levels from the binocular and monocular sections of the corresponding sections are shown in Figure 11. In most layers (e.g., layers $4 \mathrm{C}, 5,6$ ), DG uptake in the stimulated (dark) ocular dominance strips is equal to that in the adjacent binocularly stimulated region. However, in layers 2 and 3 (and marginally in layers 1 and $4 \mathrm{~B}$ ), there is actually less uptake in the binocularly stimulated region than in the stimulated monocular strips. It is not clear why an increase in neural input would lead to a decrease in DG uptake in these binocular layers, but these results do demonstrate the presence of binocular interactions in the upper striate layers.

There is a second kind of DG result that provides evidence for binocular interaction in the extragranular striate layers. In a few cases that were intended to be binocular, the DG results showed low-contrast ocular dominance strips ("semimonocular" dominance strips) in layers $4 \mathrm{Cb}$ and $4 \mathrm{~A}$ (see Fig. 12). In these cases, the low-contrast ocular bias was presumably caused by undetected optical problems in one eye. The evidence here is circumstantial, but persuasive. For instance, in a few cases, the low-contrast ocular bias appeared in DG results from a cortical region that was stimulated by a high-spatial-frequency grating, but an ocular bias in DG uptake was absent from an immediately adjacent cortical region stimulated by a low-spatial-frequency grating. Such a result is consistent with problems arising from optical aberrations. Alternatively, one might argue that the low-contrast ocular dominance strips could be evidence of binocular rivalrous suppression during periods of DG uptake. However, the presence or absence of these low-contrast ocular dominance strips in layer $4 \mathrm{Cb}$ had no obvious correlation with the degree of binocular convergence or divergence in the DG results.

At any rate, in layers beyond $4 \mathrm{~A}$ and $4 \mathrm{Cb}$, the semimonocular dominance strips in layer $4 \mathrm{Cb}$ were filled in to such an extent that one would not suspect the presence of an ocular bias in the parvocellular LGN input layers. For instance, in another case (31), we stimulated an animal with a high- (7 cycle/deg) spatialfrequency grating at a horizontal orientation, and both eyes were open and converged on the center of the screen. Nevertheless, in the autoradiographs from this animal there is a clear ocular bias in layers $4 \mathrm{~A}$ and $4 \mathrm{Cb}$ (see Fig. 13). In other layers (including $4 \mathrm{Ca}$ ), no such ocular bias is visible, and in fact there are regions in which extragranular DG strips run perpendicular to, and completely span, the underlying semimonocular dominance strips in layer $4 \mathrm{Cb}$. Presumably, the semimonocular strips are visible in $4 \mathrm{~A}$ and $4 \mathrm{Cb}$ because there is no orientation selectivity of cells or DG patterns in these layers; no orientation columns are present to override the minor optical bias. The apparent dissociation of results from magno- and parvocellular layers is reminiscent of the results described in Figure 4, and it is considered further in the Discussion.

In the description of purely monocular results, we found very little evidence of binocular input into the light ("unstimulated") ocular dominance strips in layers 2, 3, 5, and 6 (see Fig. 4). While it is difficult to be quantitative about the extent of the semimonocular "fill-in" process (Figs. 12,13), it is clearly more significant than the negligible binocular inputs demonstrated in the same extragranular layers of purely monocular cases (see Fig. 4).

The final (and most interesting) DG specialization that appears to be related to binocular disparity takes the form of an enhancement of screen region borders. The border enhancement appears as a swath of markedly increased DG uptake about 2$3 \mathrm{~mm}$ wide in striate regions corresponding to the border between adjacent patterned screen regions, or a patterned screen region and adjacent diffuse gray region, of the same or similar mean luminance (see Figs. 14, 15). The pattern of DG uptake at these borders is topographically diffuse, even when the same stimulus produces a periodic pattern of uptake in regions within the borders. The border enhancement appears after stimulation with a wide variety of patterned visual stimuli (including both grating and random-dot stimuli), but especially following blackwhite stimuli containing high-spatial-frequency components.

There are several reasons that we assume that the DG border enhancement may be related to the processing of binocular disparity. First, the border enhancement is obvious only in binocular cases. Following monocular stimulation with luminancemodulated stimuli, it was usually not present at all, and in those monocular cases in which a border enhancement could be seen, it was quite minor (see Fig. 14; cases 15, 30, 31, 37).

Second, the border enhancement occurs only in striate layers 1-3 (cf. Fig. 15, $A$ vs $B$ vs $C$ ). Within the top 3 layers, the border 

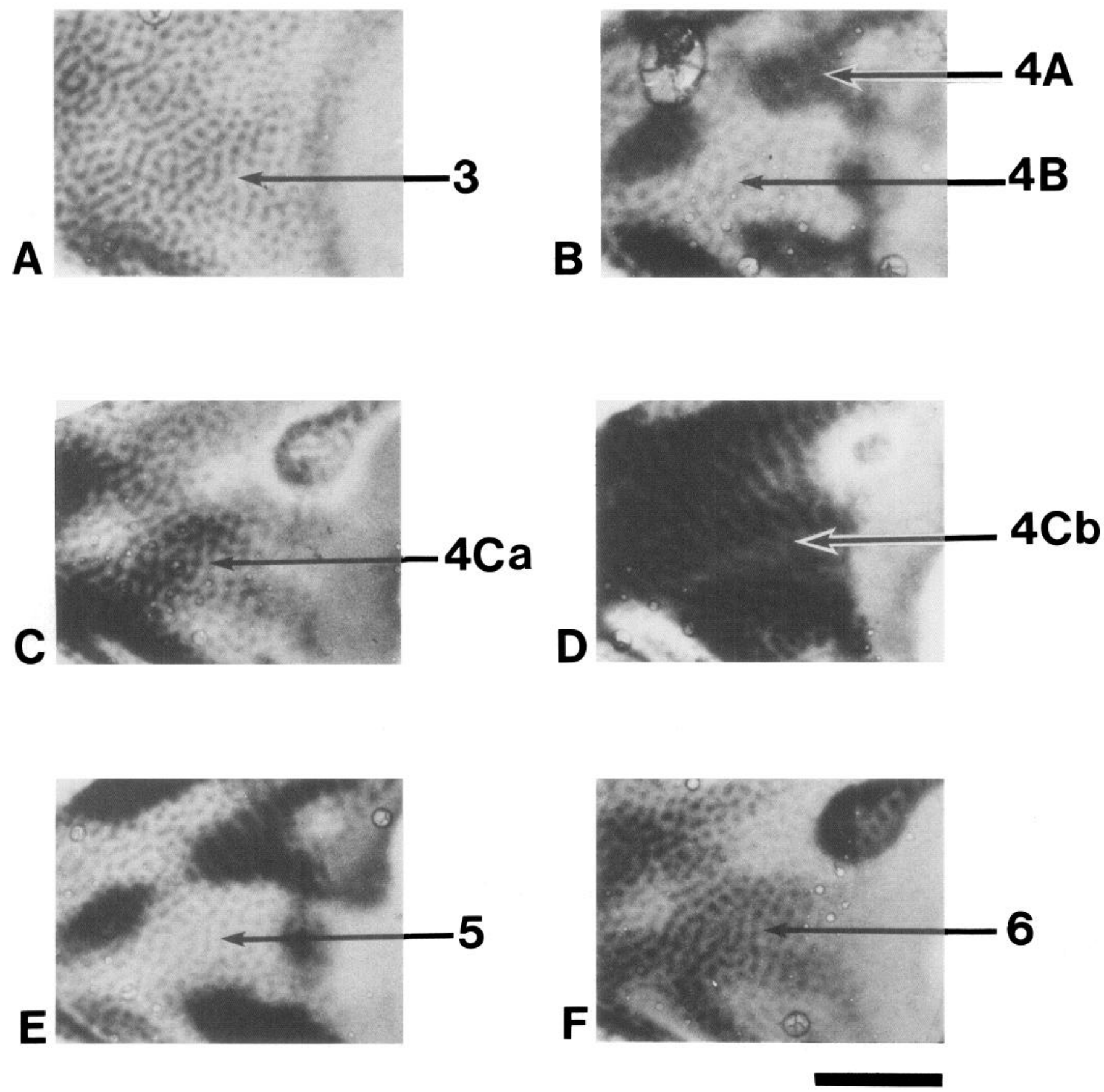

Figure 13. DG autoradiographs showing "semimonocular" dominance columns in geniculate input layers and a lack of such an ocular bias in topographically corresponding portions of other layers. The stimulus in this case was a high-spatial-frequency grating, shown at a horizontal orientation; the illustrated sections are taken from the parafoveal representation, including a visually unstimulated border region (right-hand side of $A-F$ ). The viewing conditions were intended to be binocular, but minor optical problems in one eye produced low-contrast ("semimonocular") ocular dominance strips within $4 \mathrm{Cb}(D)$ and more faintly in $4 \mathrm{~A}(B)$. The topography of the semimonocular, high-spatial-frequency orientation periodicities in other layers shows little or no indication of such an ocular bias: in front of the arrows, the orientation-specific topography crosses the semimonocular dominance strips perpendicularly, and in this region of striate cortex the DG periodicities appear to completely span the strips of weaker (lighter) ocular input in layers $4 \mathrm{Cb}$ and $4 \mathrm{~A}$. Laminae of interest are indicated in front of each arrow. One interesting aspect of these results is that the ocular imbalance appears only in layers receiving parvocellular input, such as $4 \mathrm{~A}$ and $4 \mathrm{Cb}$, but not in $4 \mathrm{Ca}$, which receives major magnocellular input. This supports other evidence indicating the presence of binocular connections in $4 \mathrm{Ca}$, but not $4 \mathrm{~A} \mathrm{or} 4 \mathrm{Cb}$.

enhancement is slightly more obvious towards the cortical surface than towards the bottom of layer 3; such a bias is unlike all other DG patterns. That is, the border enhancement remains more constant in uptake across layers $1-3$ than in other stimulus-driven patterns, so it is relatively more obvious towards the cortical surface.
When the visual stimuli are slightly misaligned on the retina, it might be supposed that the border enhancement could be due entirely to a suppression of uptake in the binocular region and a release from inhibition in the adjoining monocular region, as was experimentally demonstrated in Figure 11 . There are several lines of evidence that allow us to rule this out. First, the border 

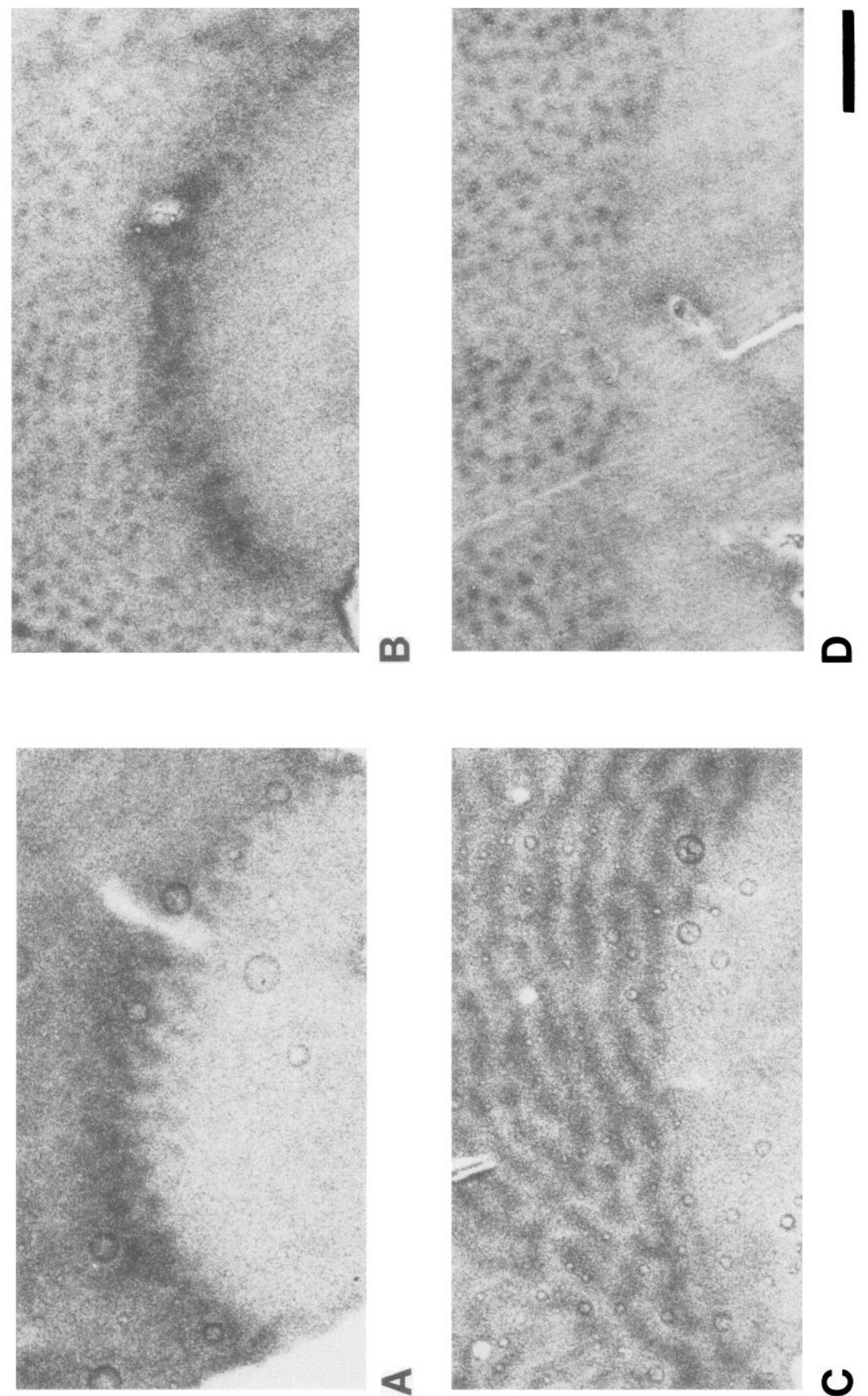



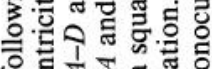
论

政

충

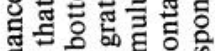

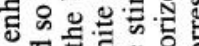

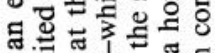

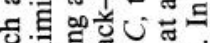

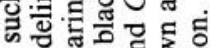
पथ

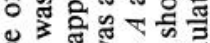

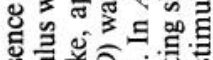

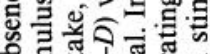

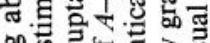

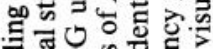
물

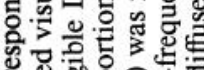
象要

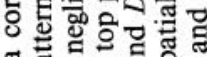
त

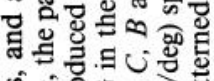
象 웡

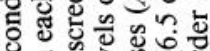
드의

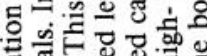
뜰

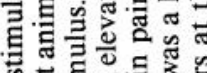
क

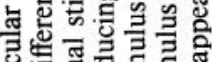

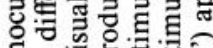

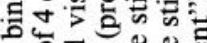

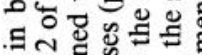
专政为 츰

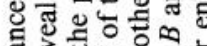

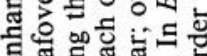

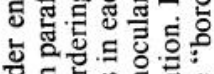

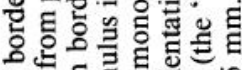
돌.

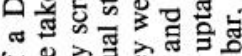

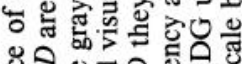
8 工

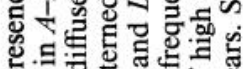

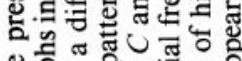
政

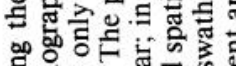
o․의

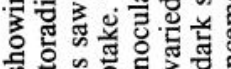

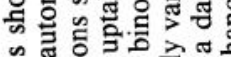

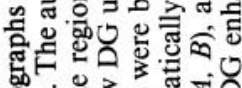
으.

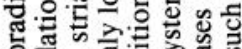

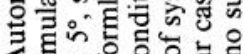

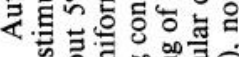

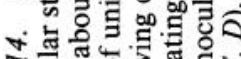

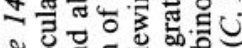

(3) 
enhancement occurs just as robustly when there is no adjoining monocular region (compare layer $4 C(B)$ with layers $2+3(A)$ in Fig. 15). Second, the border enhancement also occurs between adjacent binocularly stimulated regions, where there is again no monocular segment. Third, if the border enhancement were due to higher uptake in a monocular segment, then this enhancement should be topographically periodic rather than topographically diffuse, owing to the presence of ocular dominance columns. Finally, the laminar distribution of levels of uptake in the border enhancement is unlike that caused by DG binocular suppression, and the increase in uptake in the border enhancement greatly outstrips the relatively minor differences between monocular and binocular uptake in the DG suppression effect.

The possibility that the DG border enhancement is due to variations in mean luminance per se can also be discounted for a number of reasons. First, the border enhancement occurs about as often (and robustly) when the adjacent screen regions are set to an identical mean luminance as when they are not. Second, a known luminance mismatch between diffuse gray screen regions and diffusc gray mask regions produced no visible border in the DG autoradiographs, even when the animal was stimulated binocularly. Third, it would be logical to suppose that a border enhancement due to luminance mismatch would also appear in layer $4 \mathrm{C}$, which receives input from the relatively simple LGN receptive fields; this did not occur. Instead of being an accentuation of luminance mismatch, the border enhancement appears to be due to an active binocular mechanism that results in a delineation of visual-field regions differing from each other in pattern or texture.

\section{Baseline conditions}

In a number of animals we were able to examine the pattern of DG uptake following stimulation with time-invariant diffuse gray light. These autoradiographic results include cases in which the whole operculum was stimulated (case 2), as well as cases in which a visually unstimulated area of cortex appeared peripheral to the visually stimulated area (e.g., cases $13,18,37$ ).

Overall, levels of DG uptake in these cases were much lower than those in visually stimulated striate cortex; this much is obvious in the most peripheral portions of Figures 2 and 8 . Nonetheless, by photographing the autoradiographs at high contrast, the general pattern of DG uptake in these cases can be demonstrated and described (see Fig. 16; case 9). These particular autoradiographs are taken from a paralyzed monkey that viewed a diffuse gray stimulus of $104 \mathrm{~cd} / \mathrm{m}^{2}$. In most cases, the upper layers are quite homogenous; uptake in the cytox blob and interblob areas are indistinguishable. Layer $4 \mathrm{~A}$ is faintly visible as a thin band that is darker than $4 \mathrm{~B}$ below and slightly darker than layer 3 above. Layers $4 \mathrm{Ca}$ and $4 \mathrm{Cb}$ are the darkest layers in striate cortex and are not differentiable. Layer 5 is light, and uptake increases slightly in layer 6, although not to the levels reached in layer $4 \mathrm{C}$.

In some of these cases, the cytochrome oxidase blobs near the fovea take up slightly more DG than do the interblob areas. However, the difference in uptake between visually unstimulated blob and interblob areas near the fovea is always much less than that between stimulated and unstimulated portions of the same layer. For instance, Figure 17 shows the pattern of DG uptakc in such a case. Near the fovea, the pattern of DG uptake shows a very minor mottling due to increased relative uptake on the cytox blobs. The mottling in the DG pattern corresponding to the uniformly illuminated foveal region is essentially negligible when compared to the much higher differential uptake produced by active visual stimulation (that is, to the lines of blinking black and white squares). Quantitatively, the maximum difference we have seen between visually unstimulated fovcal blob and interblob areas is less than $5 \%$ of that seen between visually stimulated and unstimulated areas of the same layer and section.

A number of findings from other laboratories have given rise to the idea that DG uptake in the cytox blobs is much greater than that in interblob regions in a wide variety of stimulus conditions (Hendrickson and Wilson, 1979; Horton and Hubel, 1981; Humphrey and Hendrickson, 1983). However, this has not been the result in much of our autoradiographic material. Furthermore, we find that certain visual stimuli reliably produce DG uptake that is higher in the cytox interblob regions than in the blob regions (Tootell et al., 1982b). When we do see an elevated pattern of DG uptake in the parafoveal blobs, it appears to be a direct result of specific visual stimuli used in the DG experiment. Because of the importance of this issue, we were intercsted in sorting out reasons for the discrepancies between our own work and previous studies.

One of the most direct comparisons of DG uptake relative to the cytox blobs was made by Humphrey and Hendrickson (1983). These investigators found that DG injections in unparalyzed, unanesthetized squirrel monkeys produced a spotty pattern of DG uptake in the upper layers that matched that of the cytox blobs when the animals binocularly viewed a blank white screen. This is at variance with our parafoveal results from paralyzed macaques viewing similar stimuli. The discrepancy could be due to a difference between paralyzed and unparalyzed preparations, or between squirrel monkey and macaque striate architecture.

We first looked for species differences in the striate architecture. In an unpublished experiment done with Dr. Gerald Jacobs, we monocularly stimulated a paralyzed and anesthetized squirrel monkey with a retinotopically specific (but otherwise fairly general) visual stimulus. Results from layer 3 of the squirrel monkey can be seen in Figure 18 . In the squirrel monkey (which shows no anatomical evidence for ocular dominance columns), DG uptake in the stimulated region is highest in the cytox blobs, with slightly less uptake between the blobs. In the surrounding cortical region, viewing a flat gray screen, there is also a higher baseline uptake of DG in the cytox blobs. The increased DG in the squirrel monkey cytox blobs appears greater than the minor foveal effect we have seen in some macaques viewing a flat gray screen (e.g., Fig. 17), and in the squirrel monkey the effect does not appear to vary with eccentricity. The present results from paralyzed squirrel monkey match previous results from unparalyzed squirrel monkey quite well (Humphrey and Hendrickson, 1983).

In macaques viewing a very similar stimulus, however, the pattern of DG uptake is different. DG uptake within macaque cytox blobs is about the same as it is in interblob regions, both in stimulated and diffuse gray regions (Tootell et al., 1982b; see also Fig. 17). It appears, then, that at least part of the discrepancy in results between this and previous studies is due to a straightforward species difference.

In studies done before the discovery of the cytox blobs, dotlike strips of DG uptake were reported in the upper and lower layers of unparalyzed, unanesthetized macaques monocularly viewing the surrounding laboratory (Kennedy et al., 1975; Hendrickson and Wilson, 1979). The discrepancy between these previous 

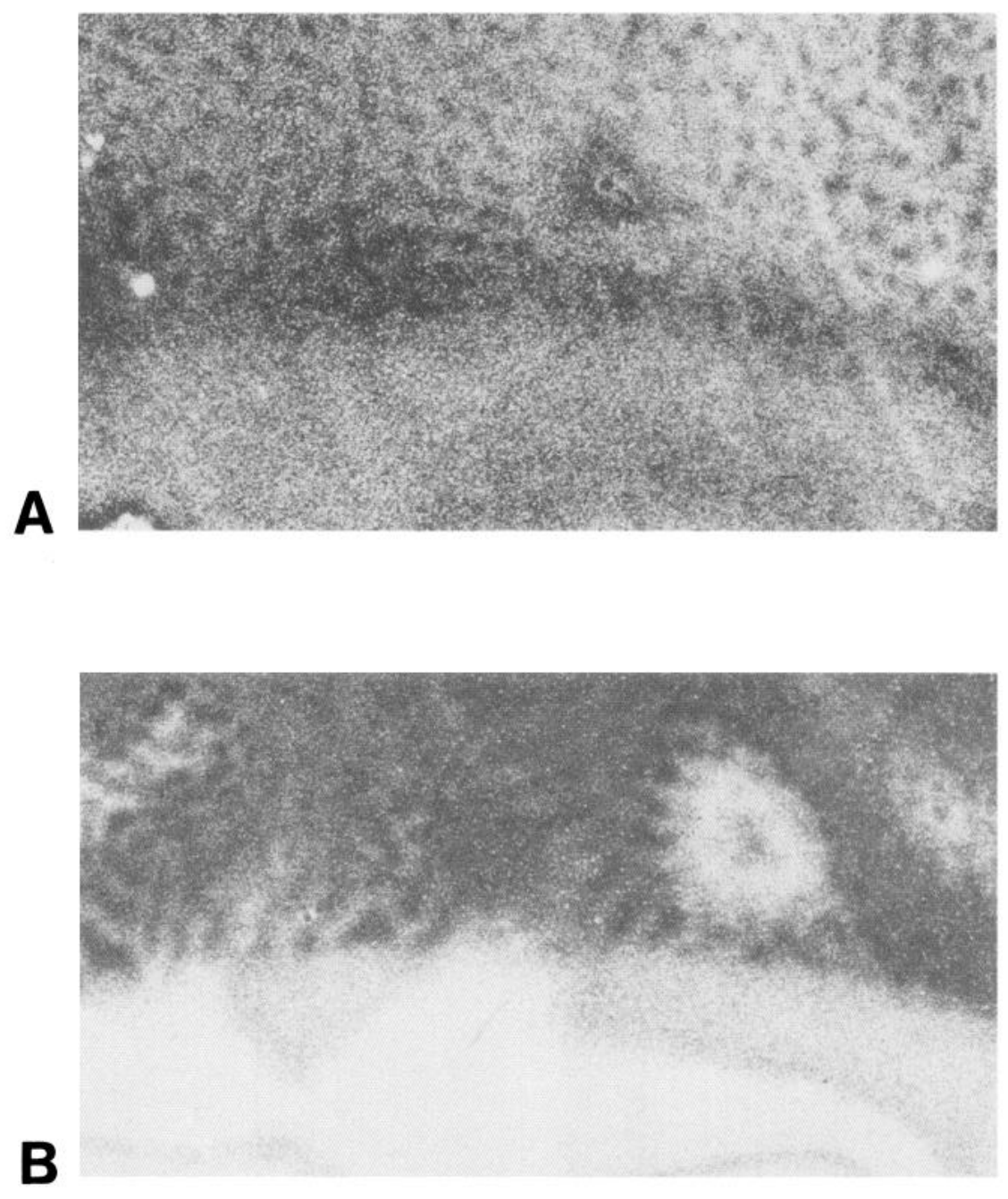

Figure 15. Laminar distribution of the DG border enhancement. $A-C$, Autoradiographs taken from topographically corresponding regions of layer $2-3$ $(A), 4 \mathrm{Ca}$ and $4 \mathrm{Cb}(B)$, and 5-6 $(C)$. The top half of $A-C$ shows the DG pattern produced by a high-spatial-frequency grating at a vertical orientation, presented binocularly. More peripheral regions of striate cortex (lower half, $A-D$ ) viewed a diffuse gray screen, of mean luminance equal to that in the stimulated region. In layers $2+3(A)$, a dark swath of high DG uptake appears at the border of patterned and diffuse visual stimulation. No such border enhancement appears in layers 4B (not shown), $4 C(B)$, or layers $5+6(C)$. Scale bar, $2.5 \mathrm{~mm}$.

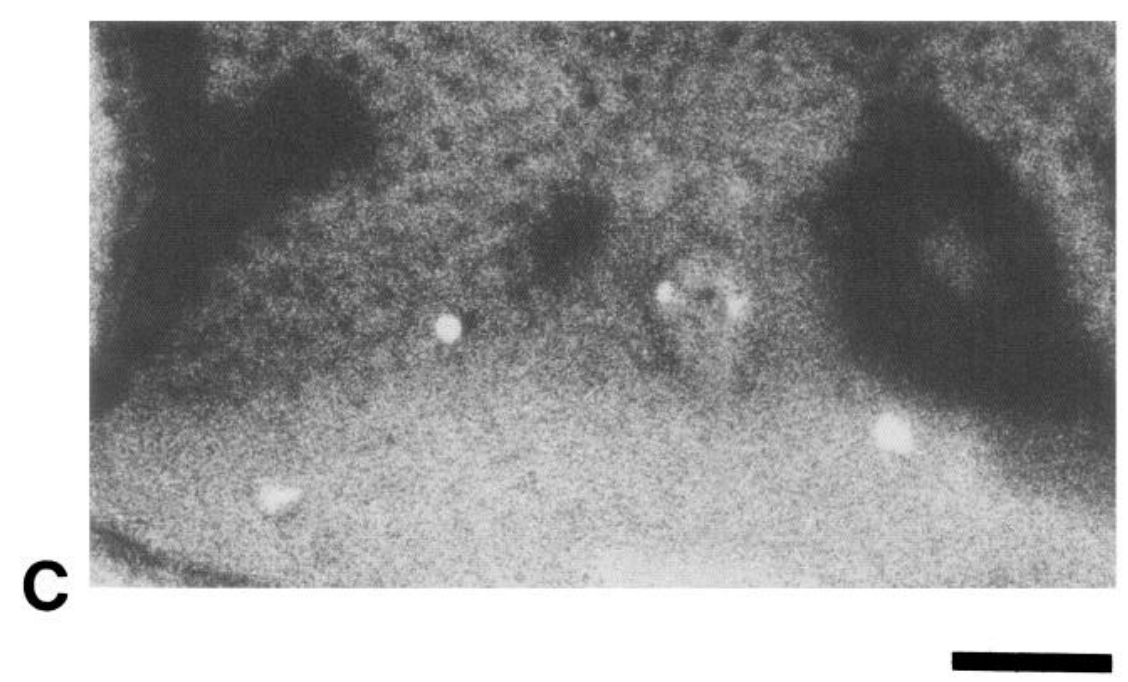

results and the present ones (see Figs. 2, 3) cannot be explained away as a species difference.

In the present studies using unparalyzed monkeys, one obvious possible explanation is that the presence of eye move- ments in itself causes a higher uptake of DG in the cytox blobs through a corollary discharge or some other mechanism. To test this idea, we injected DG into a normal macaque (case 16) in its home cage in complete darkness. The pattern of DG uptake 


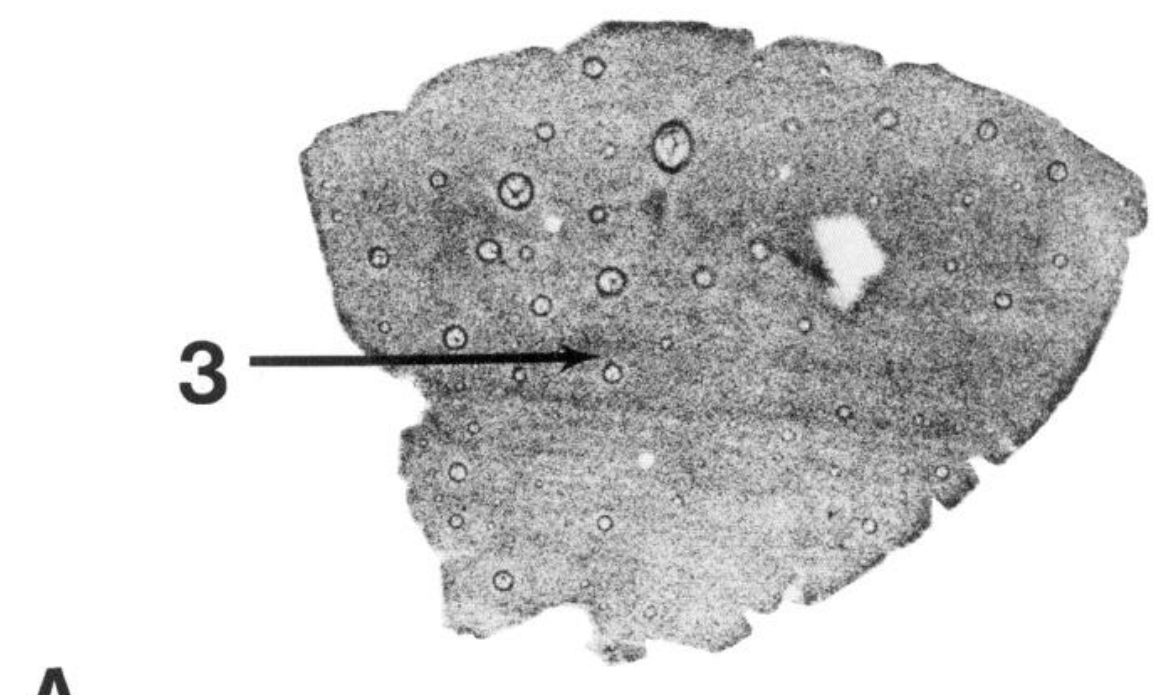

A
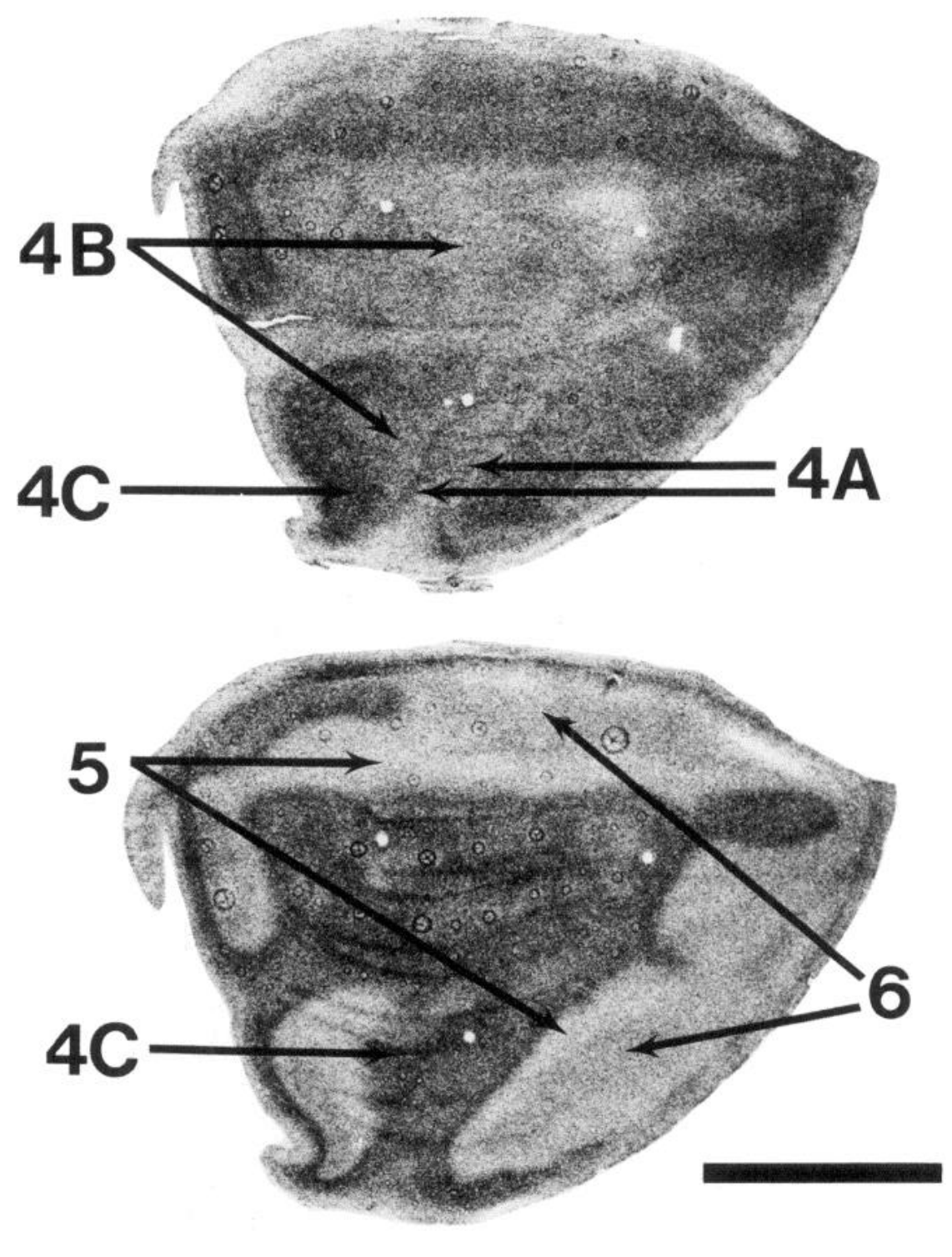

Figure 16. DG autoradiographs from a case stimulated binocularly with a diffuse gray field $\left(104 \mathrm{~cd} / \mathrm{m}^{2}\right)$. Such a stimulus produces essentially no stimulus-driven uptake; the variations visible in $A-C$ are differences in baseline uptake, photographed at high contrast to clearly reveal those minor differences in uptake that do exist. $A-C$ are taken from successively deeper sections of the same flattened operculum. The fovea is represented towards the right, the parafovea towards the left. Uptake in layers $2+3$ is essentially uniform in blob and interblob areas alike $(A)$. Uptake is high in the very thin layer $4 \mathrm{~A}$, lighter in $4 \mathrm{~B}$, and highest of all in layers $4 \mathrm{Ca}$ and $4 \mathrm{Cb}(B)$. Uptake is light again in layer 5 , and very slightly darker in layer $6(C)$. Scale bar, $1 \mathrm{~cm}$. 


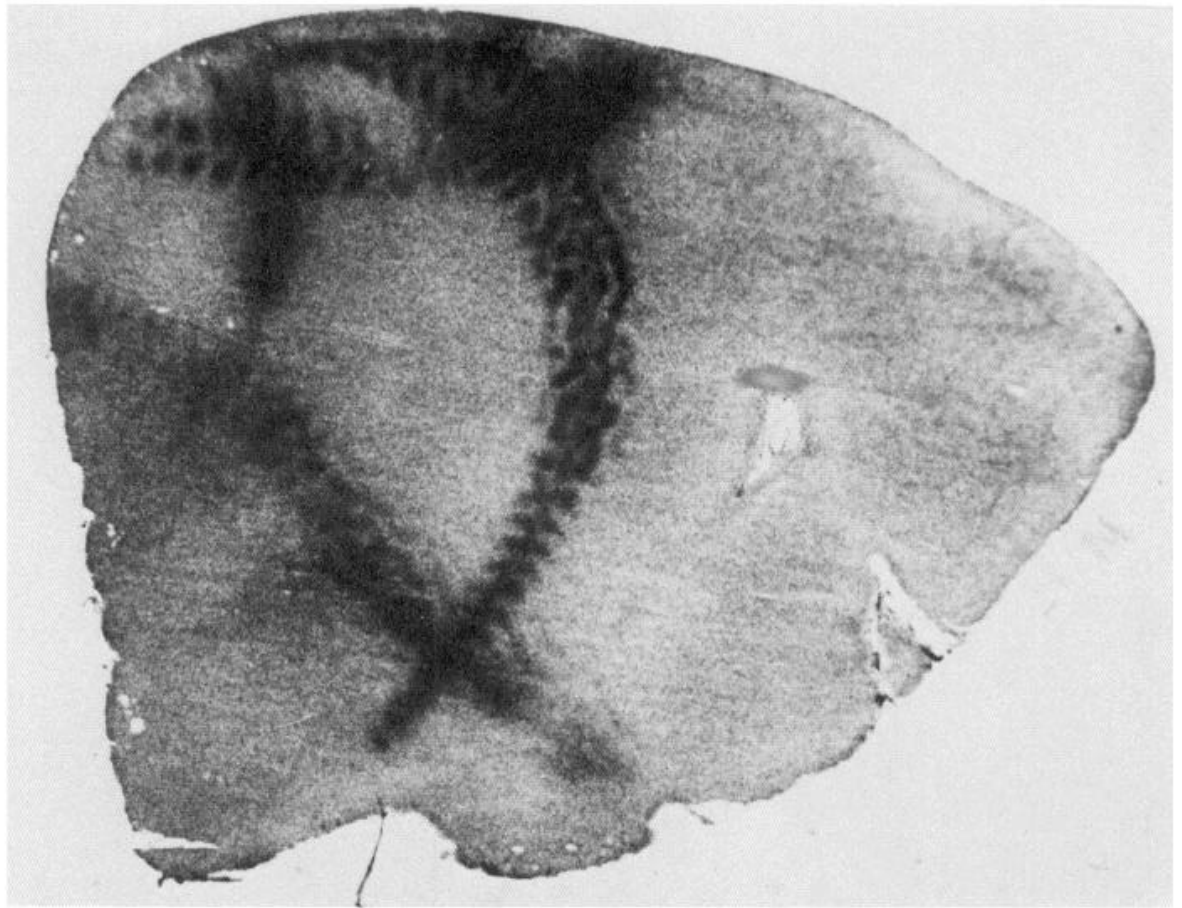

A

Figure 17. DG autoradiograph $(A)$ and corresponding section stained for cytochrome oxidase $(B)$ from layer 3 of macaque striate cortex. Towards the left in $A$, a complicated pattern of DG uptake appears, which is due to binocular visual stimulation with a retinotopically specific stimulus (described fully in Tootell et al., 1988a). At or near the foveal representation (far right, $A$ and $B$ ), striate cortex "saw" only a diffuse gray screen surrounding the retinotopic stimulus. In this and a few other cases, such a uniform gray visual stimulation condition produces very slightly higher uptake in the blobs near the fovea. However, the relative difference in levels of uptake between such foveal blob and interblob regions is an insignificant fraction of that seen in regions actively stimulated by patterned visual stimuli. Scale bar, $1 \mathrm{~cm}$.

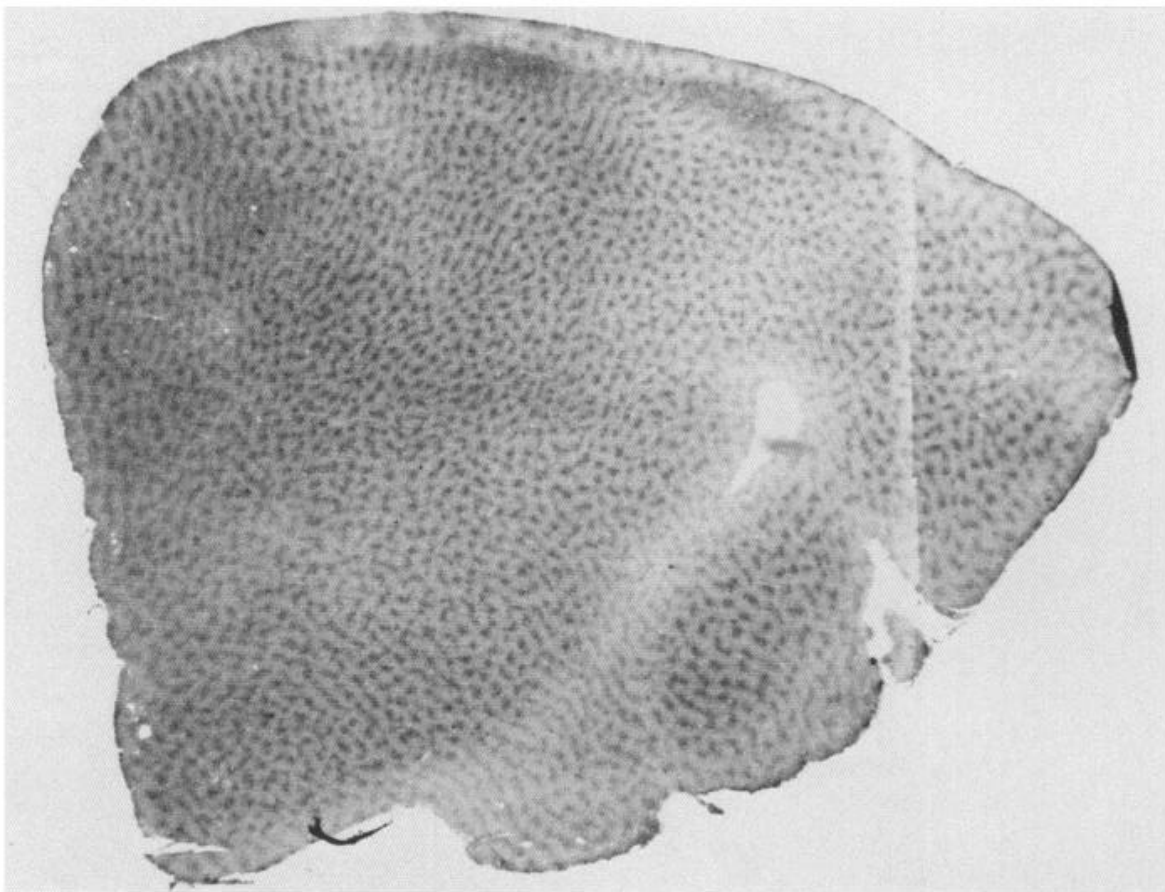

B in this animal was indistinguishable from that of paralyzed macaques viewing a diffuse gray screen (e.g., Fig. 16); a bloblike pattern of DG uptake was not visible in any layer, and the levels of uptake appeared similar to those seen in unstimulated por- tions of striate cortex in a number of cases. Thus, there do not appear to be significant differences in striate DG results that can be traced to the presence or absence of eye movements per se. This is not surprising because there appears to be little or no 
difference between the single-unit responses of striate neurons in paralyzed and unparalyzed states (Wurtz, 1969; Schiller et al., 1976).

Another possible explanation for the discrepancy between our results and the previous ones is that there was an inadvertent stimulus bias in the unparalyzed viewing conditions of the previous studies. For instance, we have found that it is easy to produce selective DG uptake in the upper-layer blobs by introducing even minor color variations into the stimulus (as occurs in many laboratories), or by removing high-spatial-frequency components (sharp contours), which might easily occur in a defocused state.

We decided to test this idea by presenting to an unparalyzed monkey a stimulus that produces high interblob uptake in a paralyzed, anesthetized monkey. We hand-held an unanesthetized, unparalyzed macaque in front of a 7.5 cycle/deg squarewave grating, shown at an orientation that was continuously varied (case 51). In studies using paralyzed monkeys, a highspatial-frequency grating produces high DG uptake in the interblobs (see Tootell et al., 1988d). This is presumably due to receptive-field sizes that are smaller in the interblobs than in the blobs (see Hubel and Livingstone, 1987). The black-white grating was printed on high-quality xerox paper and pasted to the inside of a specially constructed hemisphere. The stimulus was well lit during the time the monkey was viewing it. The hemisphere was used in an attempt to restrict the visual field to only the grating in all directions, and to keep the stimulus distance constant. It would be a mistake, however, to assume that the monkey saw only the high-spatial-frequency grating used as a stimulus. For one thing, we had no guarantee that the monkey's eyes were actually focused on the screen. Second, a number of uncontrolled variables in the stimulus introduced low-spatial-frequency components into the visual field. The hope, however, was that we could bias the visual input towards achromatic, high-spatial-frequency visual components.

The results from one operculum of this case are shown in Figure 19. Overall, the DG contrast in this case was fairly low. We presume this was due to stimulation with a high-spatialfrequency grating that was out of focus on the animal's retina for some percentage of the stimulation time; the animal was not as concerned with staring at the grating as we wished it to be. Despite the low overall contrast, some periodicity can be seen in the DG pattern towards the parafovea in layer 3 and some of the other extragranular layers (Fig. 19A). This mottling is even more obvious on the underside of the operculum, at the representation of more peripheral eccentricities (Fig. 19C). The mottled topography in Figure $19, A, C$, appears to be highest in the interblobs, as it is in the paralyzed animals. To confirm this inverse relationship it was only necessary to compare the DG and cytox topographies from the same section (see Fig. 19 and Fig. 5, $B, C$ ).

Nearer the fovea, the DG pattern is topographically uniform. This is consistent with a shift in average receptive field size with eccentricity, and with the results from paralyzed animals. In particular, the lack of uptake in the foveal cytox blobs presumably indicates a relative balance between the activity of cells in the cytox blob and interblob areas. We assume that, in the fovea, the $7.5 \mathrm{cycle} / \mathrm{deg}$ grating is closer to the mean spatial frequency tuning of all the cells. Such a pattern of uniform uptake is seen in paralyzed animals following stimulation with middle spatial frequencies at eccentricities further from the fovea. Layers $4 \mathrm{~A}$, $4 \mathrm{C}$, and 6 of this case are dark, and layers $4 \mathrm{~B}$ and 5 are quite lightly labeled.

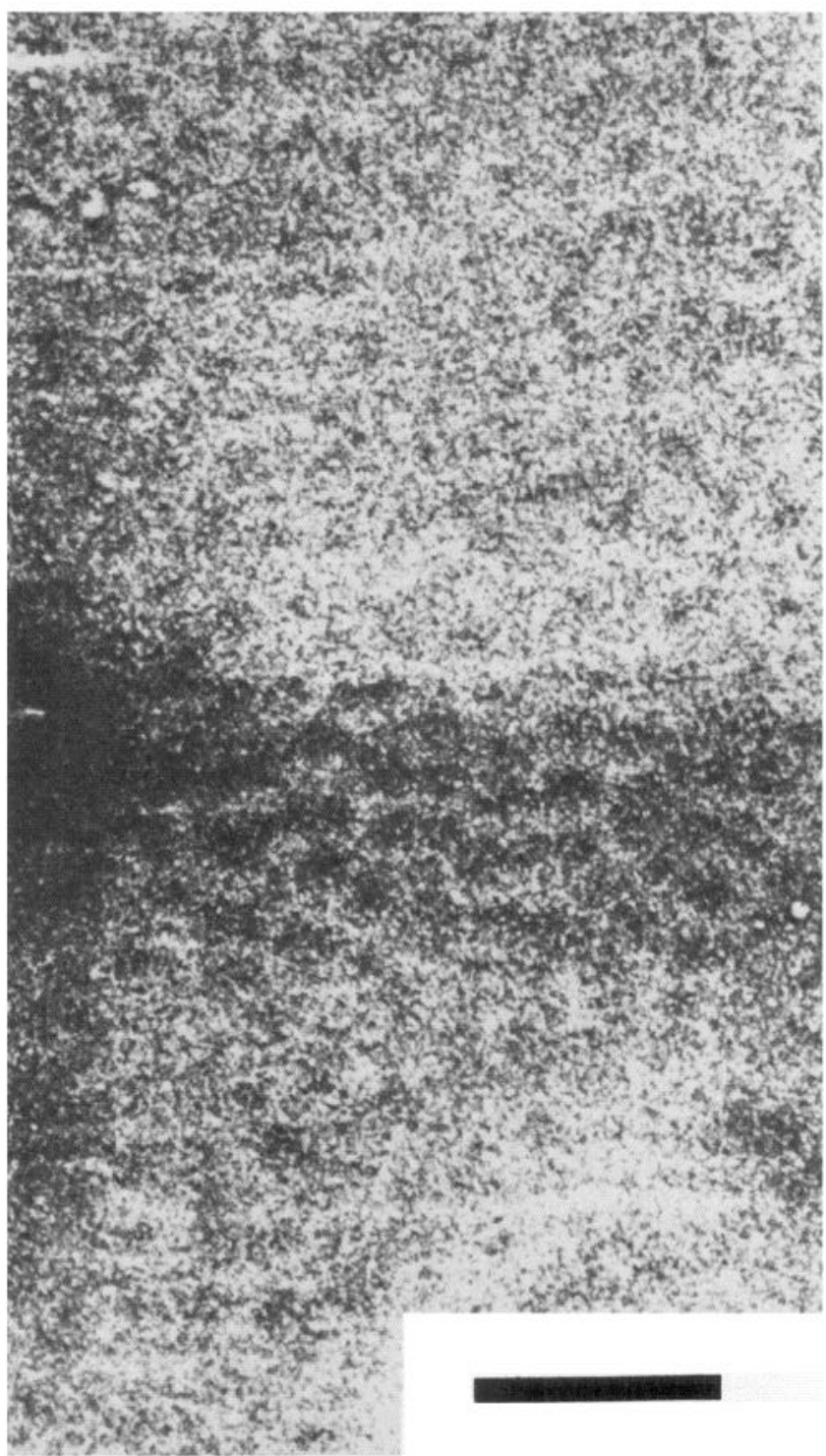

Figure 18. DG autoradiograph showing stimulus-related and stimulus-unrelated uptake in layer 3 of squirrel monkey visual cortex. Across the middle-lower portion, a region of high DG uptake was produced by a stimulus of blinking black and white checks along the horizontal meridian in the visual field. In both the stimulated and unstimulated portions of the visual field, uptake is higher in the blob than in the interblob regions. This appears to indicate a species difference between the macaque and the squirrel monkey. Scale bar, $2 \mathrm{~mm}$.

\section{Discussion}

\section{Binocular interactions}

The most intriguing results in this study are those on binocular interactions. Some of these binocular results came from careful quantitative analysis, some findings were unexpected, and some binocular interactions that we expected did not in fact show up. The results do not indicate a single immutable functional architecture, like that of orientation columns. However, this should not be too surprising, because the meshing of monocular images into a single binocular percept necessarily involves a number of diverse neural processes at once. Among these are the coding of retinal disparities into information about stereoscopic depth, the fusion of the 2 disparate images, the interface with vergence 

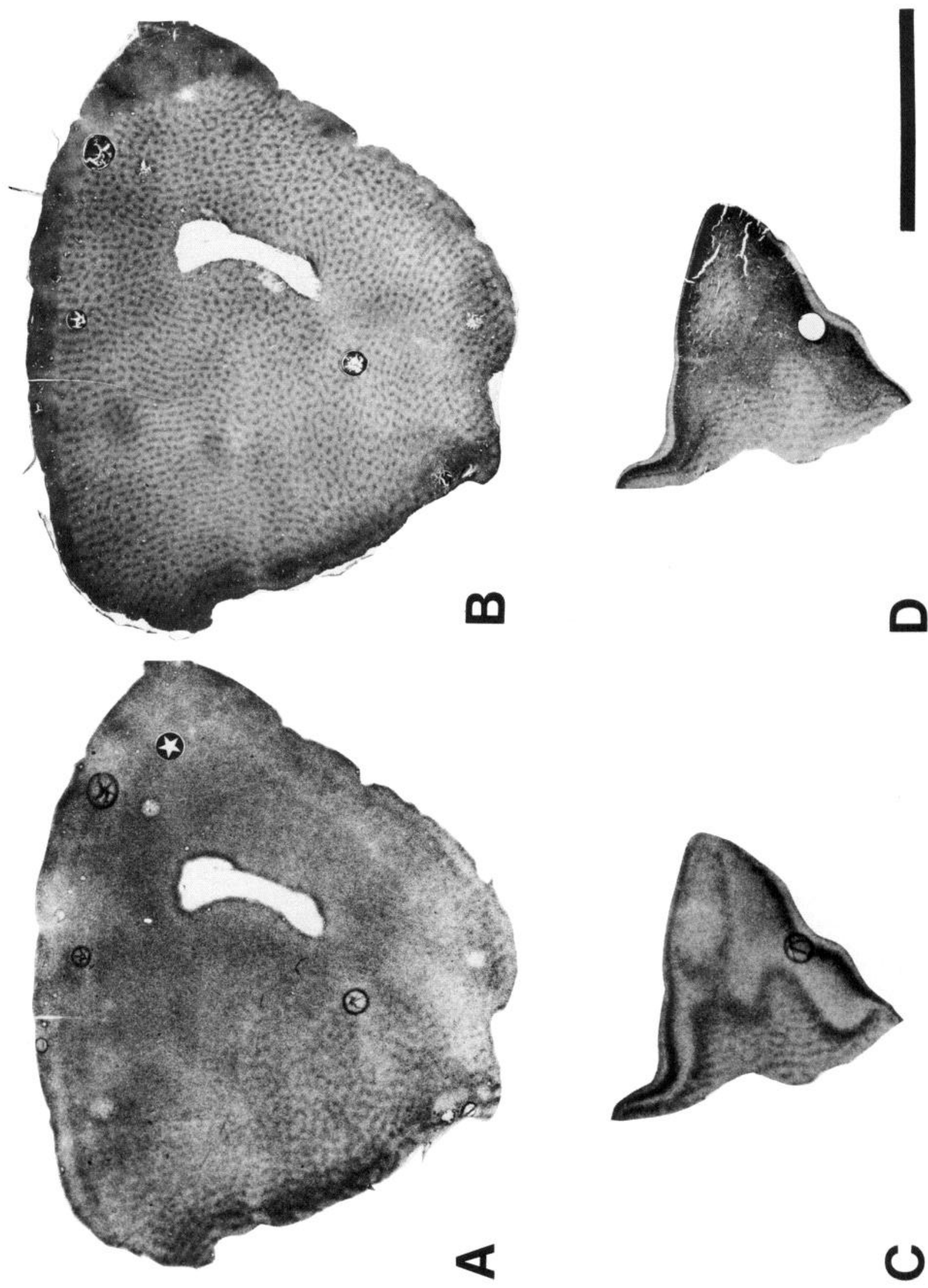

0 
eye movements, etc. Some of these processes must involve areas beyond striate cortex, but on logical grounds one would expect many of these functions to begin in striate cortex.

Because the binocular DG results fall into several different classes, they may furnish some of the data necessary to start assigning the different disparity subroutines to their respective extrastriate channels. For instance, a number of DG results indicate that the magnocellular-recipient striate layer 4Ca shows evidence of binocular intermingling that is completely absent in the parvocellular-recipient layer $4 \mathrm{Cb}$. Much of the information passing through striate layer $4 \mathrm{Ca}$ is subsequently channeled into striate layer $4 \mathrm{~B}$, and layer $4 \mathrm{~B}$ projects to a number of areas that have a high percentage of disparity-specific cells: area MT (Maunsell and Van Essen, 1983); area V3 (D. Felleman, personal communication); and the thick stripes of V2 (M. L. Livingstone and D. H. Hubel, unpublished observations). Thus, the present DG evidence for binocular mixing in layer $4 \mathrm{Ca}$ supports the general idea that binocular-specific responses are common along a magnocellular-dominated pathway.

Layer $4 \mathrm{Ca}$ looks more binocular than does layer $4 \mathrm{Cb}$ in a variety of DG contexts. In the simplest test, we showed an animal a very general visual stimulus while one eye was occluded, and compared the level of DG uptake in the resultant light ("unstimulated") ocular dominance strips with that in a nearby, binocularly unstimulated region of the same layer; this was done in different striate layers. Thus we could see whether the DG uptake produced by strictly monocular stimulation was entirely confined to the dark ("stimulated") ocular dominance strips, or whether it showed binocular spread into the other eye's dominance strips. In most striate layers, there is no evidence for spread of activity beyond the bounds of the "stimulated" ocular dominance strips, but in layer $4 \mathrm{Ca}$, monocular stimulation produces some DG uptake in the strips dominated by the closed eye (see Fig. 6).

One idea that we had is that the increased spread of DG activity in layer $4 \mathrm{Ca}$ may reflect communication between layer 4Ca cells in different eye dominance strips, and that this in turn may be an integral part of the architecture for orientation columns. After all, striate cells respond to the same orientation through either eye from the same region of the visual field, so the meshing of inputs from each eye might logically occur at, or prior to, the level at which orientation tuning becomes fixed. There are several pieces of evidence for this. First, there have been indications that orientation tuning (Blasdel and Fitzpatrick, 1984; Livingstone and Hubel, 1984) and DG orientation columns (Livingstone and Hubel, 1984; and unpublished observations) appear in the magnocellular-input layer $4 \mathrm{Ca}$. Second, the retinotopic spread of orientation-specific DG patterns is very extensive in layer $4 \mathrm{Ca}$-at least as wide as an ocular dominance strip (see Figs. 7 and 24 of Tootell et al., 1988a). Third, binocular cells have been reported in layer 4Ca (Hawken and Parker, 1984; but see also Livingstone and Hubel, 1984).

This idea is well supported by a case (shown in Fig. 14) in which an animal viewed an oriented grating clearly through one eye, and (apparently) not so clearly through the other eye. The resultant semimonocular dominance strips appear only in layer $4 \mathrm{Cb}$, and not in layer $4 \mathrm{Ca}$. The orientation-specific periodicities in layer $4 \mathrm{Ca}$ completely span the lighter semimonocular strips in underlying layer $4 \mathrm{Cb}$. Such a result strongly supports the evidence for significant binocular interactions in the magnocellular-recipient layer $4 \mathrm{Ca}$, related, perhaps, to the establishment of orientation columns. These deoxyglucose findings for binocular interactions in geniculorecipient layer $4 \mathrm{Ca}$ are also supported by an electrophysiological report of binocular cells in that layer (Hawken and Parker, 1984; but see also Livingstone and Hubel, 1984).

The other general class of DG binocular effects appears in striate layers $2+3$. Since these layers project to area V2 rather than to $V 3$ and $M T$, and since layers $2+3$ receive the strongest input from parvorecipient layer $4 \mathrm{Cb}$ rather than from magnorecipient layer $4 \mathrm{Ca}$ (e.g., Fitzpatrick et al., 1983), it is not surprising that the form of this DG binocular result in layers $2+$ 3 is different from than seen in the more magnocellular-dominated layers.

The most striking of these upper-layer effects is a swath of high DG uptake, about $2 \mathrm{~mm}$ wide, that appears at the striate representation of the stimulus borders (see Figs. 15, 16). This "border enhancement" is evenly distributed across both cytox blob and interblob portions of the upper layers. It is largely independent of differences in mean luminance between corresponding screen regions. It appears following binocular visual stimulation, particularly at the borders of a high-spatial-frequency grating, but it is not produced by monocular stimulation with visual stimuli that are otherwise identical.

The laminar distribution of the border enhancement is particularly intriguing. The enhancement does not appear in layer $4 \mathrm{C}$, which receives major geniculate input, either in $4 \mathrm{Cb}$ or in the slightly binocular $4 \mathrm{Ca}$ subdivisions. It also does not appear in the very thin layer $4 \mathrm{~A}$ nor in layer 6 , which receive minor input from the geniculate. The fact that the border enhancement is not seen in the input layers suggests that it may reflect an active cortical delineation of screen region borders.

The border enhancement is not seen in layer $4 \mathrm{~B}$ or 6 ; this rules out MT and V3 as possible destinations for the border enhancement information. Because it occurs only in layers 13 , the border enhancement is presumably a part of the V1-V2V4 stream concerned with spatial detail and color (Van Essen and Maunsell, 1983). This strict laminar and binocular spe-

$\longleftarrow$

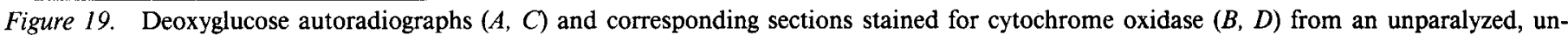

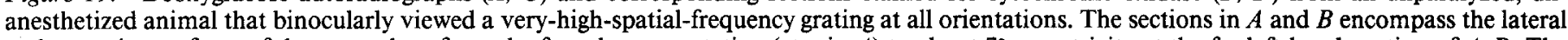

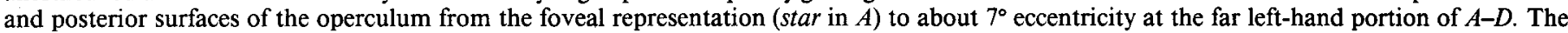

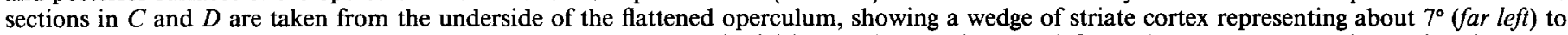

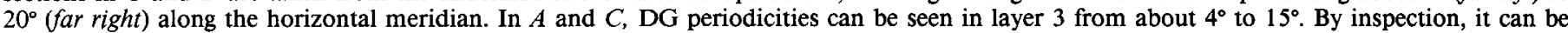

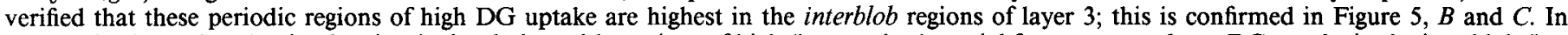

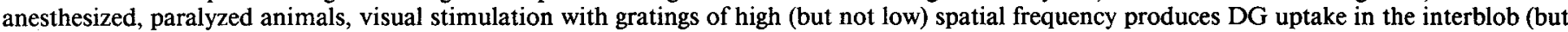

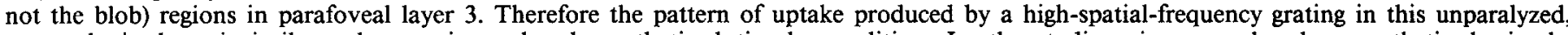

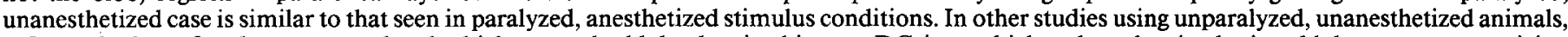

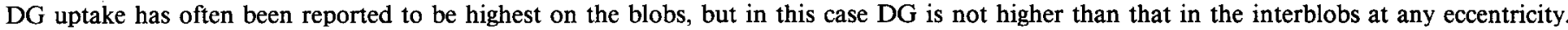
Scale bar, $1 \mathrm{~cm}$. 
cialization of the border enhancement indicates that at least some types of binocular information are completely segregated within one of the 2 extrastriate streams passing through striate cortex.

One straightforward assumption is that the DG border enhancement reflects computational processes occurring within striate layers $2+3$, which are then forwarded to V2. It is possible, however, that the border enhancement reflects a descending influence from V2 back to striate cortex. Two pieces of evidence support the centrifugal notion. First, certain aspects of the striate border enhancement are retinotopically less discrete than other retinotopic DG borders seen in layers 2 and 3 of striatc cortcx (Tootcll ct al., 1988a), and approximatcly as discrete as other DG retinotopic borders seen in area V2 (unpublished observations). Thus the retinotopic resolution of the border enhancement is more "V2-like" than "V1-like."

In addition, the DG border enhancement fades out less towards layer 1 than do other stimulus-induced DG patterns; thus the border enhancement is relatively more prominent in layers 1 and 2 than in layer 3 . This "upside-down" laminar distribution is highly reminiscent of the projections into V1 from V2, which are most prominent in striate layer 1 and decrease in strength towards layer 3 (e.g., Wong-Riley, 1978).

Some related DG data indicate that the border enhancement may itself be enhanced by (but not attributed entirely to) a suppression of DG uptake within the binocularly stimulated area. These data come from a case in which we compared the levels of DG uptake in the light and dark ocular dominance strips to lcvels in a binocularly stimulated striate region in the same hemisphere, at the same eccentricity (see Figs. 10, 11). In most layers, levels of uptake in the stimulated monocular strips were indistinguishable from those in the binocularly stimulated region. However, in the upper layers (particularly layers $2+3$ ), uptake in the binocularly stimulated region was significantly less than that in the adjacent stimulated ocular dominance strips. This is clear in both the photographs of the autoradiographs (see Fig. 10) and the quantitative measurements (see Fig. 11).

It is not clear why an increase in visual input (that is, from 2 eyes rather than one) produces a decrease in DG uptake in the upper layers; presumably, followup single-unit mapping will be able to resolve this question. Perhaps such a binocular interaction suppresses the firing of some upper-layer cells: this is the strictly empirical expectation from a host of striate DG findings that are correlated with the excitatory (but not the inhibitory) single-unit results in striate cortex. Although it is well accepted that membrane hyperpolarization (as in presynaptic inhibition) should produce increased DG uptake (e.g., Sharp, 1976; Sokoloff et al., 1977), such inhibition would reduce activity (and presumably uptake) in subsequent neurons.

The single-unit technique should also clarify the DG border enhancement in the upper layers. Electrophysiological correlates of the enhancement effect are certainly not obvious, but the effect may be related to inhibitory processes (end-stopping) that are common in receptive fields of cells in upper layers. However, the DG enhancement cannot be entirely attributed to end-stopped receptive fields as they are presently understood, since this type of inhibition would produce the border enhancement in monocular cases as well as in binocular cases, and this does not occur (see Fig. 14). Furthermore, end-stopped cells are numerous in other layers besides layers $2+3$ (Schiller et al., 1976), and the wide laminar distribution of end-stopped cells does not correlate well with the strict laminar compartmentalization of the DG border enhancement.

In recordings from awake, behaving macaques, Poggio (1984) has reported that each of the various kinds of disparity-specific cells can be found in each striate layer, although the relative percentage of each type varies somewhat. Tuned inhibitory cells are more common in the upper striate layers than in the lower striate layers, and this may conceivably explain some of the binocular DG effects seen in the upper layers. However, this type of cell is relatively rare, even in the upper layers. It may be necessary to test the disparity-specific responses of single cells with stimuli similar to those used in the present DG experiments in order to resolve the relationship of the upper-layer DG interactions to single-unit disparity specificities.

Poggio and co-workers have also shown that the majority of cells in macaque striate cortex respond selectively to the absolute disparity of binocularly presented stimuli (Poggio and Fischer, 1977; Poggio and Talbot, 1981; Poggio, 1984). Each cell responds uniquely, but the different response types can be grouped into about 4 different classes. Because this is so, we initially expected that the absolute binocular disparity at which our stimuli were presented would strongly affect the DG results. [It should be emphasized that the absolute stimulus disparity could be established (post hoc) in these DG experiments with a degree of certainty that is impossible to obtain in single-unit studies from paralyzed animals simply by measuring the size of the monocular segment at a known eccentricity in the DG representation of the stimulus borders.] In careful control experiments, we were able to show that the absolute disparity at which we presented the stimuli had no obvious effect on the DG results, within a reasonable range of disparities near zero. This lack of DG sensitivity to absolute disparity within a binocularly stimulated region made the interpretation of subsequent DG work much simpler.

A priori, one might have expected that the manifold varieties of disparity-class cells would bias the DG results in one way or another, depending on the absolute binocular disparity of the stimulus. It is not clear why a disparity organization was not more obvious in the DG results. One obvious possibility is that disparity tuning is in fact relatively uncommon in striate cortical responses from paralyzed animals, as has been found by some investigators (e.g., Hubel and Wiesel, 1968, 1970).

Another possibility is that cells of various disparity classes are not functionally grouped in striate cortex. However, given the necessity for coding binocular disparities at the very first binocular levels (and its computational complexity), it would be surprising if cells of various disparity classes were not topographically grouped to some extent in striate cortex, although this is not obvious in our DG tests. It is possible that the grain of the disparity grouping is too fine for current DG techniques, or that elucidation of a disparity architecture will require $D G$ mapping with awake, behaving animals trained to fixatc and converge at different planes in the visual field.

\section{Baseline conditions}

In several animals, we measured DG uptake during various baseline visual stimulation conditions. These included stimulation with a diffuse gray screen, complete darkness, and with gratings of systematically varied orientation and spatial frequency. Different stimuli were tested in paralyzed and unparalyzed animals, and in New and Old World monkeys. These 
various baseline manipulations were geared towards establishing a norm against which the effects of more specific stimuli could be judged. When we had obtained enough of the baseline results to see a general pattern, it became obvious that the pattern we saw was not the pattern that others before us had seen. Gradually, a picture began to emerge that rationalized most or all of the available results.

In some previous papers, regions of high DG uptake were attributed to a generally higher metabolic activity in the cytochrome oxidase blobs (e.g., Horton and Hubel, 1981; Humphrey and Hendrickson, 1983). In the present study, this pattern of high blob uptake was also seen, but in our results, high uptake in the cytox blobs was almost entirely stimulus-driven and stimulus-specific. We find that both stimulus-driven and stimulusunrelated DG uptake in the cytox blobs is often about equal to that in the interblobs and (in at least one stimulus condition) higher in the cytox interblobs than in the cytox blobs.

We tried to sort out the reasons for the discrepancy between results in a systematic way. In several previous studies, DG activity was sampled in unparalyzed, unanesthetized monkeys; it was therefore conceivable that neural activity related to eye movements caused higher uptake in the cytox blobs. Thus we tested 2 unanesthetized, unparalyzed monkeys (1) in complete darkness, and (2) viewing a high-spatial-frequency grating at all orientations. In neither of these cases was there the highest DG uptake on the blobs at any eccentricity. The pattern of uptake in these cases was indistinguishable from that in cases in which a paralyzed, anesthetized monkey viewed a similar stimulus.

These results are comforting in 2 respects. First, the unambiguous lack of high blob uptake in the unparalyzed, unanesthesized cases eliminates one possible confounding variable between the results from different laboratories. Specifically, it allows us to assume that the high blob uptake seen in other studies was not due to their use of unparalyzed animals (Kennedy et al., 1975; Humphrey and Hendrickson, 1983). More generally, these results shore up the evidence that striate functional activity in the paralyzed primate can be directly generalized to normal (psychophysical) viewing conditions. This is important because the great bulk of neurophysiological evidence is based on results from paralyzed animals, while psychophysical results are, of course, taken from unparalyzed humans and animals.

Some of the most detailed evidence for a higher uptake on the cytochrome oxidase blobs comes from results in the squirrel monkey (Humphrey and Hendrickson, 1983), and we find a similar result in our own tests from a number of squirrel monkeys (Fig. 17, and unpublished observations). In the squirrel monkey, uptake was generally higher in the blobs than in the interblobs, but in the macaque this was generally not so. Thus, some of the discrepancy between results appears to be due to a species difference, and the results of Humphrey and Hendrickson (1983) are generally not in conflict with those of the present study. Such a species difference is not particularly surprising, since there are significant differences between the striate architecture (e.g., Hendrickson and Wilson, 1979), the physiology (e.g., Jacobs, 1983), and the visual capacity (De Valois and Jacobs, 1968) of the squirrel monkey and the macaque monkey.

Some portion of the remaining discrepancy may be attributed to differences in the nature of the stimuli used in the present and previous studies. In the case illustrated in Figure 19, an unanesthetized, unparalyzed animal was shown a high-spatialfrequency grating at all orientations, which was pasted on the inside of a specially constructed hemisphere. The high-spatialfrequency bias of the stimulus produced high DG uptake in the parafoveal interblob regions, just as it does in paralyzed animals (Tootell et al., 1982b, 1983, 1988d). In other cases, stimulated with color-varying or luminance-varying stimuli of low spatial frequency, uptake was highest in the parafoveal blobs (Tootell and Silverman, 1981; Tootell et al., 1982b, 1983).

Although it is hard to tell from some previous studies, it may also be possible that previous investigators obtained patterns of high uptake in the cytox blobs by sampling near the fovea; we also see such a DG pattern there. The foveal blob bias in these DG results may be attributed largely (but not entirely) to the known decrease in average size of receptive fields closer to the fovea, relative to the constant (and relatively low) spatial frequency range of these stimulus patterns.

A final point bears on the relationship of DG uptake to cytochrome oxidase activity. We have shown that certain stimuli produce DG uptake that is highest in the light (interblob) regions of layer 3 (see Fig. 19 and Tootell et al., 1982b, 1983, 1988d), and in related studies we show that DG uptake can be eliminated selectively from either the blob or interblob regions by manipulating the choice of stimulus (see Tootell et al., 1982b, 1988b, c). How can these results be rationalized with a cytochrome oxidase architecture that is essentially invariant in normal animals?

There are a number of possibilities. First, the cytochrome oxidase may be completely unrelated to functional activity. At least the strong form of this idea is directly contradicted by the evidence: a number of strictly visual manipulations produce weak changes in the levels of cytochrome oxidase in striate cortex (e.g., Horton, 1984). There is also some evidence to support a weaker form of the idea: the cytochrome oxidase blobs can be seen well before birth, a time when patterned visual input is lacking (Horton, 1984). It might be argued that differences in the levels of cytochrome oxidase activity could be traced to differences in spontaneous activity in the parent cell population (e.g., Wong-Riley and Carrol, 1984). However, if such differences were directly traceable to differences in spontaneous activity, they would likely show up in the DG results, and they do not.

Another possibility is that the cytochrome oxidase activity simply indicates regions of striate cortex that have high visually driven activity in the normal visual environment. If we assume that the time-averaged input from a "normal" visual environment has a lot of color (and perhaps low-spatial-frequency luminance) cues, then the time-averaged DG pattern one might expect from such an environment is very similar to that seen in the cytochrome oxidase architecture. However, color-specific DG uptake is also strong in the lower-layer blobs, which stain only faintly for cytochrome oxidase. Also, this does not explain the robust cytochrome oxidase staining patterns in animals caged for months in an achromatic environment. Therefore, it is unlikely that cytochrome oxidase activity simply reflects timeaveraged differences in the visual stimulus load handled by striate cortex.

\section{Methodological considerations}

In the original DG protocol (Sokoloff et al., 1977), it was recommended that tissue not be perfused or fixed, on the assumption that the metabolic products of DG were water-soluble. 
Following these histological procedures, DG label is found predominantly in the neuropil (e.g., Sharp, 1976).

However, it has since been shown that much of the DG label rcmains in the tissue following perfusion and fixation with aldehydes (Des Rosiers and Descarries, 1978). This indicates some incorporation of the label into a water-insoluble product. The labeled, water-insoluble fraction appears to include glycogen, which is presumably stored in the cell soma (Durham et al., 1981; Kai-Kai and Penreath, 1981; Penreath et al., 1982; Witkovsky and Yang, 1982; Nelson et al., 1984). In tissue prepared in this way, the DG label is strikingly localized to cell bodies, in addition to labeling the background of other neural compartments (Durham et al., 1981; Kai-Kai and Penreath, 1981; Pilgrim and Wagner, 1981; Witkovsky and Yang, 1982; Hökfelt et al., 1983; Durham and Woolsey, 1985).

It is thus not clear how much our data represent activity in neuron cell bodies, and how much they represent activity in neuropil. We have often adopted the working hypothesis that our data reflect activity in striate cortical cells, rather than in the neuropil. There are several reasons why we take this position.

First of all, it is far easier to test the idea that cells in a given region act like the DG than it is to test whether the neuropil acts like the DG. Not only is it extremely difficult to record from neuropil, but the neuropil itself is a (hopelessly) complex amalgam of wandering processes without easily definable boundaries. So we adopt the cell-body assumption because it is a testable null hypothesis.

Second, in a number of cases we compared autoradiographs produced by the same tissue section, before and after an extended aqueous rinse, and clearing with xylene and ethanol. Such a rinse would presumably increase the proportion of labeling from cell bodies by rinsing out label from the neuropil. Though $80-85 \%$ of our ${ }^{14} \mathrm{C}$ label was lost through such a procedure, the remaining label was adequate to produce autoradiographs, and the labeling patterns in these rinsed autoradiographs did not differ significantly from those produced by our usual procedure. Though this does not necessarily indicate that DG patterns in our usual procedure are derived from cell bodies per se, it does indicate that at this level of magnification, few discrepancies may be found between results from the present series of studies and those from stricter labeling of cell bodies.

Third, there are a number of instances in which the presynaptic inputs to a given striate region arc functionally different from the cell bodies in that region, and in these instances the DG so far reflects the function of the cell bodies. For instance, in the macaque, unit recording from cells in the geniculorecipient layer 4Ca shows orientation tuning and orientation columns (Blasdel and Fitzpatrick, 1984; Livingstone and Hubel, 1984), and this is matched by prominent DG orientation columns in this layer (Livingstone and Hubel, 1984, and unpublished observations). The DG evidence here is especially telling, because here the DG must be reflecting the activity of the (oriented) layer 4Ca cells, as opposed to the activity of the (nonoriented) LGN afferents terminating within layer $4 \mathrm{Ca}$. The demonstration of double-opponency in many upper-layer blob cells (Livingstone and Hubel, 1984) has also been matched by DG results showing essentially the same thing (Tootell et al., 1988b).

In other comparisons, the DG and the electrophysiology have also correlated nicely; indeed, there is not yet a single documented discrepancy between data from the 2 measures. In the tree shrew, the topography of orientation columns appears similar in single-unit and DG tests (Humphrey and Norton, 1980; Humphrey et al., 1980). In cat striate cortex, units and DG patterns show orientation preferences at the same place (Schoppmann and Stryker, 1981). DG and unit-mapping measurements of variation in spatial frequency preference in the cat have also correlated well (Silverman, 1984).

Even if the detailed concordance between single-unit and DG measures breaks down at some (future) point, it would be shortsighted to disregard the wealth of architectural detail available with the DG technique. It can, in fact, be argued that the most interesting areas in striate cortex will turn out to be areas in which the DG and unit-activity measures reliably differ. Consider a hypothetical cortical cell whose dendrites receive concurrent (and equally strong) excitatory and inhibitory inputs. By current assumptions, this pattern of inputs will produce no net change in the firing rate of the postsynaptic cell body (measured by the single-unit electrode), but there will be high DG uptake in the immediate area due to high activity in the synapses and dendrites. If a discrepant example like this can be found, it will not invalidate the DG technique, but rather serve as a conceptual fulcrum for prying open the intrinsic neural circuitry for examination. Such instances should produce a more complete understanding of the exact cellular compartments at which various striate receptive-field transformations take place. However, at the present moment in history, we are awaiting the demonstration of a single discrepancy between the DG and unit techniques in visual cortex.

\section{References}

Blakemore, C. (1970) The representation of three-dimensional visual space in the cat's striate cortex. J. Physiol. (Lond.) 209: 155-178.

Blasdel, G. G., and D. Fitzpatrick (1984) Physiological organization of layer 4 in macaque striate cortex. J. Neurosci. 4: 880-895.

Blasdel, G. G., and J. S. Lund (1983) Termination of afferent axons in macaque striate cortex. J. Neurosci. 3: 1389-1413.

Clarke, P. G. H., I. M. L. Donaldson, and D. Whitteridge (1976) Binocular visual mechanisms in cortical areas I and II of the sheep. J. Physiol. (Lond.) 256: 509-526.

Daniel, P. M., and D. Whitteridge (1961) The representation of the visual field on the cerebral cortex in monkeys. J. Physiol. (Lond.) 159: 203-221.

Des Rosiers, M. H., and L. Descarries (1978) Adaptation de la méthode au déoxyglucose à l'échelle cellulaire; préparation histologique du système nerveux central en vue de le radio-autographie à haute résolution. C. R. Acad. Sci. (Paris) Ser. D 287: 153.

De Valois, R. L., and G. H. Jacobs (1968) Primate color vision. Science 162: 518-523.

Durham, D., and T. A. Woolsey (1985) Functional organization in cortical barrels of normal and vibrissae-damaged mice: A $\left({ }^{3} \mathrm{H}\right)$ 2-deoxyglucose study. J. Comp. Neurol. 235: 97-1 10.

Durham, D., T. A. Woolsey, and L. Kruger (1981) Cellular localization of $2-\left[{ }^{3} \mathrm{H}\right]$ dcoxy- $d$-glucose from paraffin-embedded brains. J. Neurosci. 1: 519-526.

Ferster, D. (1981) A comparison of binocular depth mechanisms in areas 17 and 18 of the cat visual cortex. J. Physiol. (Lond.) 311:623655.

Fitzpatrick, D., K. Itoh, and I. T. Diamond (1983) The laminar organization of the lateral geniculate body and the striate cortex in the squirrel monkey (Saimiri sciureus). J. Neurosci. 3: 673-702.

Gattass, R., C. G. Gross, and J. H. Sandell (1981) Visual topography of V2 in the macaque. J. Comp. Neurol. 201: 519-539.

Goochee, C., W. Rasband, and L. Sokoloff (1980) Computerized densitometry and color coding of $\left[{ }^{14} \mathrm{C}\right]$ deoxyglucose autoradiographs. Ann. Neurol. 7: 359-370.

Haseltine, E. C., E. J. DeBruyn, and V. A. Casagrande (1979) Demonstration of ocular dominance columns in Nissl-stained sections of monkey visual cortex following enucleation. Brain Res. 176: 153158.

Hawken, M. J., and A. J. Parker (1984) Contrast sensitivity and orientation selectivity in lamina IV of the striate cortex of Old World monkeys. Exp. Brain Res. 54: 367-372. 
Hendrickson, A. E., and J. R. Wilson (1979) A difference in $\left[{ }^{14} \mathrm{C}\right]$ deoxyglucose autoradiographic patterns in striate cortex between $\mathrm{Ma}$ caca and Saimiri following monocular stimulation. Brain Res. 170 : 353-358.

Hendrickson, A. E., S. P. Hunt, and J. Y. Wu (1981) Immunocytochemical localization of glutamic acid decarboxylase in monkey striate cortex. Nature 292: 605-607.

Hockfield, S., R. D. McKay, S. H. C. Hendry, and E. G. Jones (1983) A surface antigen that identifies ocular dominance columns in the visual cortex and laminar features of the lateral geniculate nucleus. Cold Spring Harbor Symp. Quant. Biol. 48: 877-889.

Hökfelt, T., C. B. Smith, A. Peters, G. Norell, A. Crane, M. Brownstein, and L. Sokoloff (1983) Improved resolution of the 2-deoxy-d-glucose technique. Brain Res. 289: 311-316.

Horton, J. C. (1984) Cytochrome oxidase patches: A new cytoarchitectonic feature of monkey cortex. Phil. Trans. R. Soc. Lond. [Biol.] 304: 199-253.

Horton, J. C., and E. T. Hedley-Whyte (1984) Mapping of cytochrome oxidase patches and ocular dominance columns in human visual cortex. Phil. Trans. R. Soc. Lond. [Biol.] 304: 255-272.

Horton, J. C., and D. H. Hubel (1981) Regular patchy distribution of cytochrome oxidase staining in primary visual cortex of macaque monkey. Nature 292: 762-764.

Hubel, D. H., and Livingstone, M. L. (1987) Segregation of form, color and stereopsis in primate area 18. J. Neurosci. 7: 3378-3415.

Hubel, D. H., and T. N. Wiesel (1968) Receptive fields and functional architecture of monkey striate cortex. J. Physiol. (Lond.) 195: 215243.

Hubel, D. H., and T. N. Wiesel (1970) Cells sensitive to binocular depth in area 17 of the macaque monkey cortex. Nature 225: $41-42$.

Hubel, D. H., and T. N. Wiesel (1972) Laminar and columnar distribution of geniculo-cortical fibers in the macaque monkey. J. Comp. Neurol. 158: 267-294.

Hubel, D. H., and T. N. Wiesel (1977) Functional architecture of macaque monkey visual cortex. Ferrier lecture. Proc. R. Soc. Lond. [Biol.] 198: 1-59.

Hubel, D. H., T. N. Wiesel, and S. LeVay (1974) Visual field of representation in layer IVC of monkey striate cortex. Soc. Neurosci. Abstr. 1974: 264.

Humphrey, A. L., and A. E. Hendrickson (1983) Background and stimulus-induced patterns of high metabolic activity in the visual cortex (area 17) of the squirrel and macaque monkey. J. Neurosci. 3 : $345-358$.

Humphrey, A. L., and T. T. Norton (1980) Topographic organization of the orientation column system in the striate cortex of the tree shrew (Tupai glis) I. Microelectrode recording. J. Comp. Neurol. 192: 531547.

Humphrey, A. L., L. C. Skeen, and T. T. Norton (1980) Topographic organization of the orientation column system in the striate cortex of the tree shrew (Tupai glis) II. Deoxyglucose mapping. J. Comp. Neurol. 192: 549-566.

Jacobs, G. H. (1983) Within-species variation in visual capacity among squirrel monkeys (Saimiri sciureus): Sensitivity differences. Vision Res. 23: 239-248.

Kai-Kai, M. A., and V. W. Penreath (1981) High resolution analysis of $\left({ }^{3} \mathrm{H}\right) 2$-deoxyglucose incorporation into neurons and glial cells in invertebrate ganglia: Histological processing of nervous tissue for selective marking of glycogen. J. Neurocytol. 10:693-708.

Kennedy, T. C., M. H. Des Rosiers, M. Reivich, F. Sharpe, and L. Sokoloff (1975) Mapping of functional neural pathways by autoradiographic survey of local metabolic rate with [14C] deoxyglucose. Science 187: 850-853.

LeVay, S., D. H. Hubel, and T. N. Wiesel (1975) The pattern of ocular dominance in macaque visual cortex revealed by a reduced silver stain. J. Comp. Neurol. 159: 559-576.

LeVay, S., M. Connally, J. Houde, and D. C. Van Essen (1985) The complete patterns of ocular dominance stripes in the striate cortex and visual field of the macaque monkey. J. Neurosci. 5: 486-50I.

Livingstone, M. S., and D. H. Hubel (1984) Anatomy and physiology of a color system in the primate visual cortex. J. Neurosci. 4: 309356.

Marr, D., and T. Poggio (1979) A computational theory of human stereo vision. Proc. R. Soc. Lond. [Biol.] 204: 301-328.

Maunsell, J. H. R., and D. C. Van Essen (1983) Functional properties of ncurons in middle temporal visual area of the macaque monkey II: Binocular interactions and sensitivity to binocular disparity. $J$. Neurophysiol. 49: 1148-1167.
Merrill, E. G., and A. Ainsworth (1972) Glass-coated platinum plated tungsten microelectrodes. Med. Biol. Eng. 10: 662-672.

Nelson, T., E. E. Kaufman, and L. Sokoloff (1984) 2-Deoxyglucose incorporation into rat brain glycogen during measurement of local cerebral glucose utilization by the 2-deoxyglucose method. J. Neurochem. 43: 949-956.

Penreath, V. W., L. H. Seal, and M. A. Kai-Kai (1982) Incorporation of $\left({ }^{3} \mathrm{H}\right) 2$-deoxyglucose into glycogen in nervous tissues. Neuroscience 7: $759-767$.

Pilgrim, C. H., and H.-J. Wagner (1981) Improving the resolution of the 2-deoxy-D-glucose method. J. Histochem. 29: 190-194.

Poggio, G. F. (1984) Processing of stereoscopic information in primate visual cortex. In Dynamic Aspects of Neocortical Function, G. M. Edelman, W. E. Gall, and W. M. Cowan, eds., pp. 613-634, Wiley, New York.

Poggio, G. F., and B. Fischer (1977) Binocular interaction and depth sensitivity in striate and prestriate cortex of behaving rhesus monkeys. J. Neurophysiol. 40: 1392-1405.

Poggio, G. F., and W. H. Talbot (1981) Mechanisms of static and dynamic stereopsis in foveal cortex of the rhesus monkey. J. Physiol. (Lond.) 315: 469-492.

Schiller, P. H., B. L. Finlay, and S. L. Volman (1976) Quantitative studies of single-cell properties in monkey striate cortex. I. Spatiotemporal organization of receptive fields. J. Neurophysiol. 39: 12881319.

Schoppmann, A., and M. P. Stryker (1981) Physiological evidence that the 2-deoxyglucose method reveals orientation in cat visual cortex. Nature 293: 574-576.

Schwartz, W. J., C. B. Smith, L. Davidsen, H. Savaki, L. Sokoloff, M. Mata, D. J. Fink, and H. Gainer (1979) Metabolic mapping of functional activity in the hypothalamo-neurohypophyseal system in the rat. Science 205: 723-725.

Sharp, F. R. (1976) Relative cerebral glucose uptake of neuronal perikarya and neuropil determined with 2-deoxyglucose in resting and swimming rat. Brain Res. 110: 127-139.

Silverman, M. S. (1984) Deoxyglucose and electrophysiological evidence for spatial frequency columns in cat striate cortex. Ph.D. thesis, University of California, San Francisco.

Sokoloff, L., M. Keivich, C. Kennedy, M. H. Des Rosiers, C. S. Patlak, K. D. Pettigrew, O. Sakurada, and M. Shinohara (1977) The [14C] deoxyglucose method for measurement of local cerebral glucose utilization theory, procedure, and normal values in the conscious and anesthesized rat. J. Neurochem. 28: 897-916.

Stanski, D. R., D. J. Greenblatt, and E. Lowenstein (1978) Kinetics of intravenous and intramuscular morphine. Clin. Pharmacol. Ther. 24: $52-59$.

Switkes, E., R. B. H. Tootell, M. S. Silverman, and R. L. De Valois (1986) Picture processing techniques applied to autoradiographic studies of visual cortex. J. Neurosci. Methods 15: 269-280.

Tootcll, R. B. H., and M. S. Silverman (1981) A comparison of cytochrome oxidase and deoxyglucose patterns in macaque visual cortex. Soc. Neurosci. Abstr. 7: 356.

Tootell, R. B. H., and M. S. Silverman (1985) Two methods for flatmounting cortical tissue. J. Neurosci. Methods 15: 177-190.

Tootcll, R. B. H., M. S. Silverman, E. Switkes, and R. L. De Valois (1982a) Deoxyglucose analysis of retinotopic organization in primate striate cortex. Science 218: 902-904.

Tootell, R. B. H., M. S. Silverman, E. Switkes, and R. L. De Valois (1982b) Organization of cortical modules. Soc. Neurosci. Abstr. 8 707

Tootell, R. B. H., M. S. Silverman, R. L. De Valois, and G. H. Jacobs (1983) Functional organization of the second cortical visual area of primates. Science 220: 737-739.

Tootell, R. B. H., S. L. Hamilton, E. Switkes, and R. L. De Valois (1985) DG as a "functional HRP" in macaque striate cortex. Invest. Ophthalmol. Vis. Sci. (Suppl.) 26: 8.

Tootell, R. B. H., E. Switkes, M. S. Silverman, and S. L. Hamilton (1988a) Functional anatomy of macaque striate cortex. II. Retinotopic organization. J. Neurosci. 8: 1531-1568.

Tootell, R. B. H., M. S. Silverman, S. L. Hamilton, R. L. De Valois, and F. Switkes (1988b) Functional anatomy of macaque striate cortex. III. Color. J. Neurosci. 8: 1569-1593.

Tootell, R. B. H., S. L. Hamilton, and E. Switkes (1988c) Functional anatomy of macaque striate cortex. IV. Contrast and magno-parvo streams. J. Neurosci. 8: 1594-1609.

Tootell, R. B. H., M. S. Silverman, S. L. Hamilton, E. Switkes, and R. 
L. De Valois (1988d) Functional anatomy of macaque striate cortex. V. Spatial frequency. J. Neurosci. 8: 1610-1624.

Van Essen, D. C., and J. H. R. Maunsell (1983) Hierarchical organization and functional streams in the visual cortex. Trends Neurosci. 6: $370-375$.

Wiesel, T. N., D. H. Hubel, and D. M. K. Lam (1974) Autoradiographic demonstration of ocular dominance columns in the monkey striate cortex by means of transneuronal transport. Brain Res. 79: 273-279.

Witkovsky, P., and C.-Y. Yang (1982) Uptake and localization of ${ }^{3} \mathbf{H}$ 2-deoxy-D-glucose by retinal photoreceptors. J. Comp. Neurol. 204: $105-116$.
Wong-Riley, M. (1978) Reciprocal connections between striate and prestriate cortex in squirrel monkey as demonstrated by combined peroxidase histochemistry and autoradiography. Brain Res. 147: 159164.

Wong-Riley, M., and E. W. Carroll (1984) Effect of impulse blockage on cytochrome oxidase activity in monkey visual system. Nature 307 : 262-264.

Wurtz, R. H. (1969) Visual receptive fields of striate cortex neurons in awake monkey. J. Neurophysiol. 32: 727-742. 